\title{
STREAMLINE-BASED PRODUCTION DATA INTEGRATION IN NATURALLY FRACTURED RESERVOIRS
}

\author{
A Dissertation \\ by \\ MISHAL HABIS AL HARBI \\ Submitted to the Office of Graduate Studies of \\ Texas A\&M University \\ in partial fulfillment of the requirements for the degree of \\ DOCTOR OF PHILOSOPHY
}

May 2005

Major Subject: Petroleum Engineering 
STREAMLINE-BASED PRODUCTION DATA INTEGRATION IN

NATURALLY FRACTURED RESERVOIRS

\author{
A Dissertation \\ by \\ MISHAL HABIS AL HARBI \\ Submitted to the Office of Graduate Studies of \\ Texas A\&M University \\ in partial fulfillment of the requirements for the degree of \\ DOCTOR OF PHILOSOPHY
}

Approved as to style and content by:

Akhil Datta-Gupta

(Chair of Committee)

Hans Juvkam-Wold

(Member)
Daulat D. Mamora

(Member)

Bani Mallick

(Member)

Steve A. Holditch

(Head of Department)

May 2005

Major Subject: Petroleum Engineering 


\begin{abstract}
Streamline-Based Production Data Integration in Naturally Fractured Reservoirs.

(May 2005)

Mishal Habis Al Harbi,

B.S., King Fahd University of Petroleum and Minerals, Saudi Arabia;

M.S., Stanford University

Chair of Advisory Committee: Dr. Akhil Datta-Gupta
\end{abstract}

Streamline-based models have shown great potential in reconciling high resolution geologic models to production data. In this work we extend the streamline-based production data integration technique to naturally fractured reservoirs. We use a dualporosity streamline model for fracture flow simulation by treating the fracture and matrix as separate continua that are connected through a transfer function. Next, we analytically compute the sensitivities that define the relationship between the reservoir properties and the production response in fractured reservoirs. Finally, production data integration is carried out via the Generalized Travel Time inversion (GTT). We also apply the streamline-derived sensitivities in conjunction with a dual porosity finite difference simulator to combine the efficiency of the streamline approach with the versatility of the finite difference approach. This significantly broadens the applicability of the streamlinebased approach in terms of incorporating compressibility effects and complex physics.

The number of reservoir parameters to be estimated is commonly orders of magnitude larger than the observation data, leading to non-uniqueness and uncertainty in reservoir parameter estimate. Such uncertainty is passed to reservoir response forecast which needs to be quantified in economic and operational risk analysis. In this work we sample parameter uncertainty using a new two-stage Markov Chain Monte Carlo (MCMC) that is very fast and overcomes much of its current limitations. The computational efficiency comes through a substantial increase in the acceptance rate during MCMC by using a fast linearized approximation to the flow simulation and the likelihood function, the critical link between the reservoir model and production data. 
The Gradual Deformation Method (GDM) provides a useful framework to preserve geologic structure. Current dynamic data integration methods using GDM are inefficient due to the use of numerical sensitivity calculations which limits the method to deforming two or three models at a time. In this work, we derived streamline-based analytical sensitivities for the GDM that can be obtained from a single simulation run for any number of basis models. The new Generalized Travel Time GDM (GTT-GDM) is highly efficient and achieved a performance close to regular GTT inversion while preserving the geologic structure. 


\section{DEDICATION}

To my beloved parents, to my dear wife for her patience, to my dear brother, Fawaz, for his inspiration and support, and to my dear father in-law, my family and my in-laws for all their care and support. 


\section{ACKNOWLEDGMENTS}

All praise and gratitude goes to Allah for providing me with patience and for blessing me with His endless bounties.

I would like to express my deep gratitude to Saudi Aramco for their financial support and for allowing me to pursue my Ph.D. degree. I would also like to express my gratitude to my advisor and committee chairman, Dr. Akhil Datta-Gupta, for his invaluable advice, academic guidance and personal support throughout my years of study.

I would like to thank Dr. H. Juvkam-Wold, Dr. D. Mamora, and Dr. B. Mallick for serving as committee members and for their valuable comments and suggestions.

I want to also thank my Saudi Aramco advisor, Brad Brumfield, for his tireless effort in supporting Saudi Aramco students.

Special thanks goes to my friends in the reservoir characterization group, Dr. Zhong He (now with Schlumberger), Dr. Adel Mallah (now with Kuwait University), Ahmad Al-Huthali (Now with Saudi Aramco), Dr. Ahmad Dawoud (now with Schlumberger), Dr. Sang Heon Lee (now with Chevron Texaco), Dr. Arun Kharghoria (now with Petrotel), Dr. Leonardo Vega (now with Schlumberger), Adedayo Oyerinde, Edwardo Jimenez, Dr. Hao Cheng, , Ichiro Osaka, Cheng Wu Yuan, Xianlin Ma, Sandeep Kaul, Fady Chaban, Deepak Devegowda, and Elkin Arroyo for their helpful discussions, friendship and making my graduate years memorable.

Last but not least, I would like to thank my dear friends in our Saudi student community. Their friendship has made College Station a second home for all of us. 


\section{TABLE OF CONTENTS}

Page

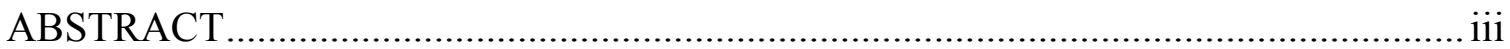

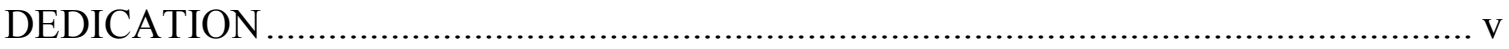

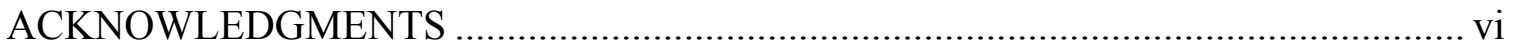

TABLE OF CONTENTS .................................................................................. vii

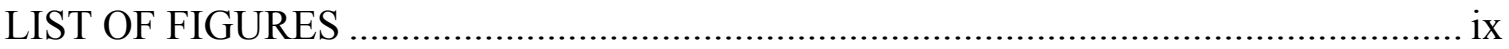

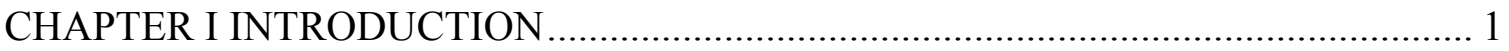

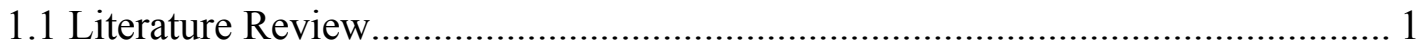

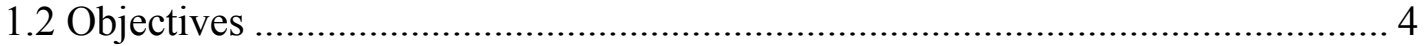

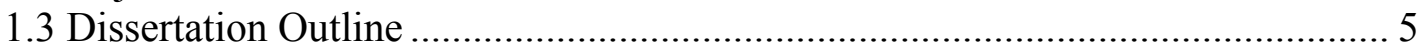

CHAPTER II APPROACH TO PARAMETER ESTIMATION IN FRACTURED

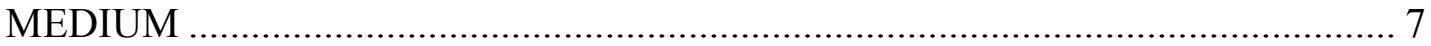

2.1 Dual Porosity Fracture Flow Simulation ......................................................... 7

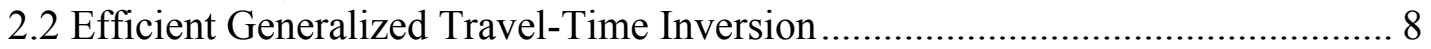

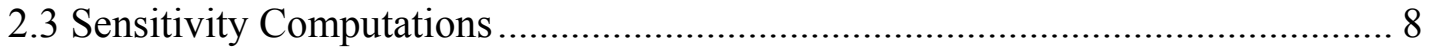

2.4 Data Integration and Objective Function Regularization ................................ 9

CHAPTER III MATHEMATICAL FORMULATION .............................................. 11

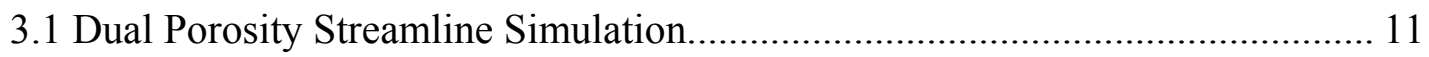

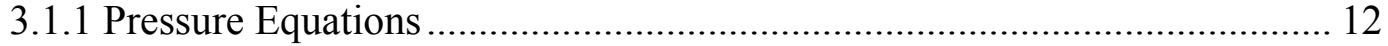

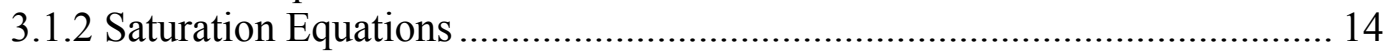

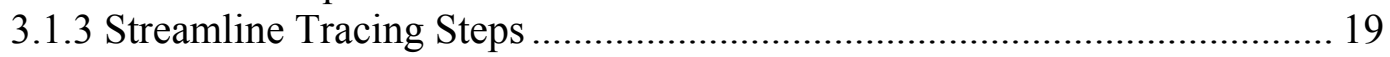

3.1.4 Pressure and Streamlines Updating ..................................................... 22

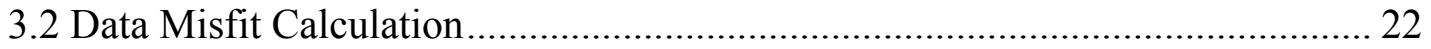

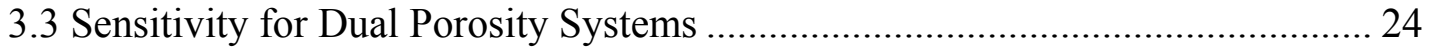

3.3.1 Dual Porosity Analytical Sensitivities .................................................... 25

3.3.2 Verification of Dual Porosity Sensitivity............................................... 27

3.3.3 Accounting for Changing Field Conditions ........................................... 29

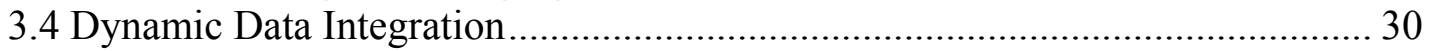

CHAPTER IV APPLICATION OF DUAL POROSITY INVERSION......................... 32

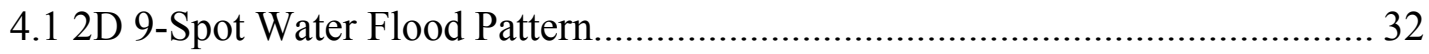

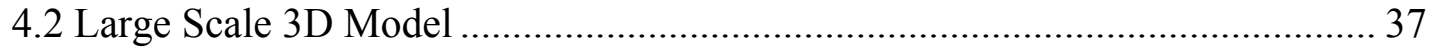


4.2.1 Prior Model-1: 50\% Fracture Information ................................................ 43

4.2.2 Prior Model-2 : 75\% Fracture Information ............................................. 49

CHAPTER V UNCERTAINTY ASSESSMENT USING APPROXIMATE MCMC .... 55

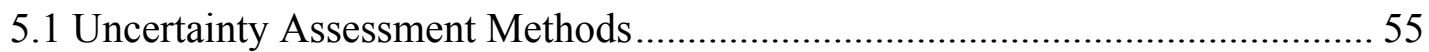

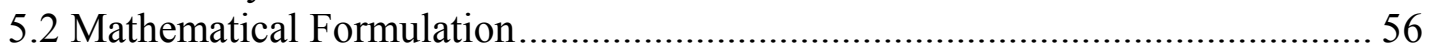

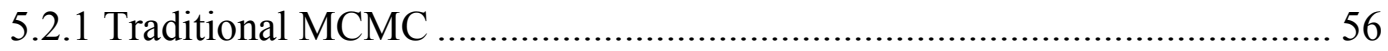

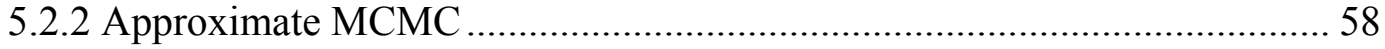

5.2.3 Parameter Perturbation Using Gradual Deformation................................... 59

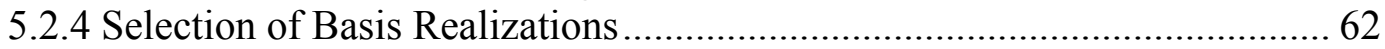

5.3 Approximate MCMC Workflow................................................................... 68

CHAPTER VI APPROXIMATE MCMC: FIELD APPLICATIONS ............................. 71

6.1 Approximate and Full MCMC Performance Comparison................................... 71

6.2 2D Example : Quarter 5-spot Waterflood......................................................... 77

6.3 3D Example: Goldsmith Field Case ………………………………………..... 83

CHAPTER VII GENERALIZED TRAVEL TIME INVERSION USING GRADUAL

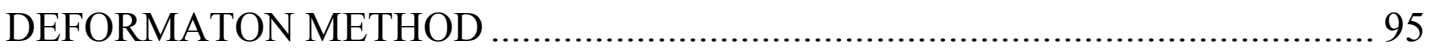

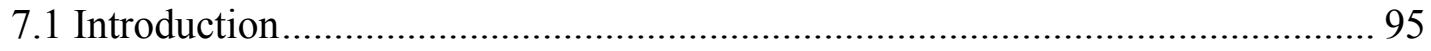

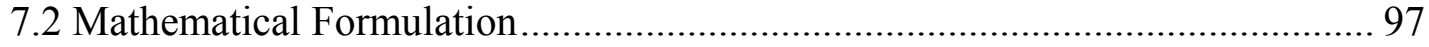

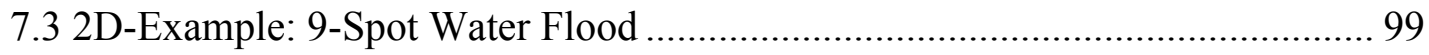

CHAPTER VIII CONCLUSIONS AND RECOMMENDATIONS …………………... 105

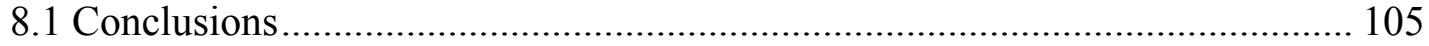

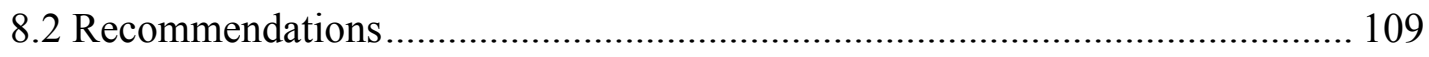

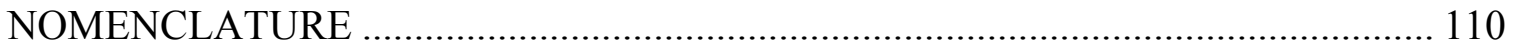

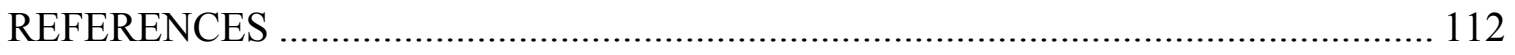

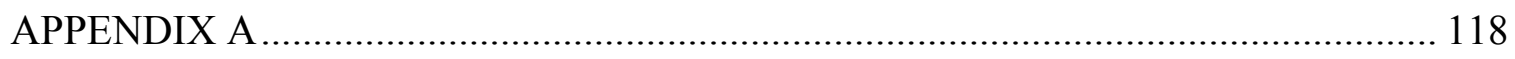

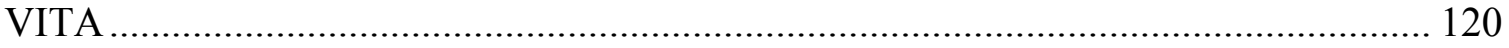




\section{LIST OF FIGURES}

FIGURE

3.1 Dual-porosity single-permeability (DPSP) system ..........................................12

3.2 Streamline and time of flight calculations ..........................................................20

3.3 Saturation evolution along streamlines - single and dual porosity examples

3.4 Illustration of generalized travel-time shift.

3.5 Comparison of numerical and analytical sensitivities in

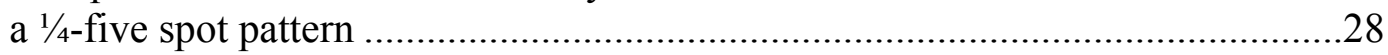

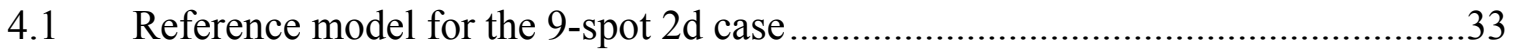

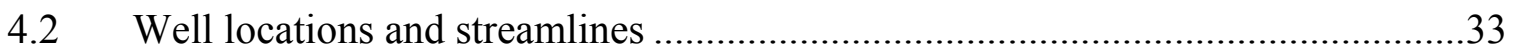

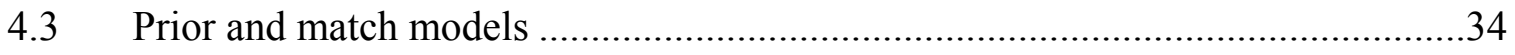

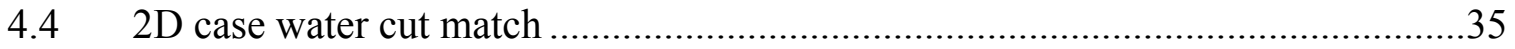

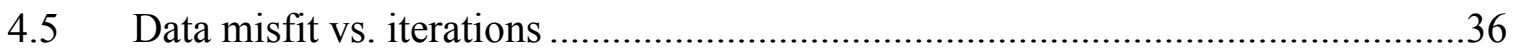

4.6 Reference fracture permeability distribution ...................................................38

4.7 Discrete fracture layers converted to permeability (left panel) using fracture intensity

4.8 Relative permeability for matrix and fracture systems .........................................40

4.9 (a) Top view shows well locations and streamlines at the end of the last update. (b) 3D streamlines traverse layers in 3D space

4.10 Discrete fracture networks for 3 different layers with $50 \%$ fracture information.....

4.11 Permeability distribution with $50 \%$ fracture information

4.12 Water cut match and initial response for 30 wells.

Almost all the wells showed better water cut match after inversion 
FIGURE

4.13 Two layers illustrating that integration of water cut data re-established permeability contrast and identified major flow paths while preserving the prior information

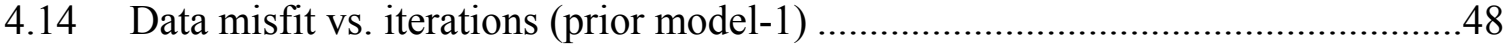

4.15 Discrete fracture network for 3 layers with $70 \%$ fracture information.................49

4.16 Permeability distribution for the prior model with $75 \%$

fracture information

4.17 Water cut match and initial response for 30 wells for prior model-2

4.18 Two layers illustrating changes to the prior model for matching production data. Note that much of the prior model remains unchanged to preserve geologic realism

4.19 Misfit vs. number of iterations (prior model-2) .............................................54

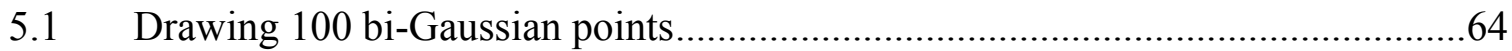

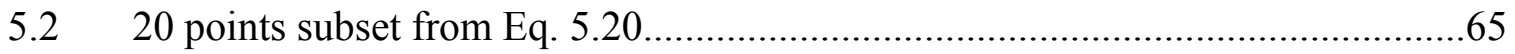

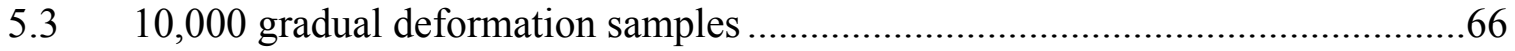

5.4 Objective function maximization for optimum subset....................................68

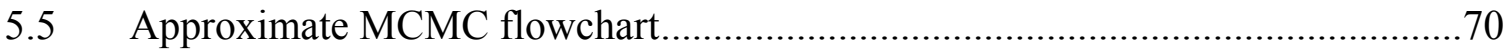

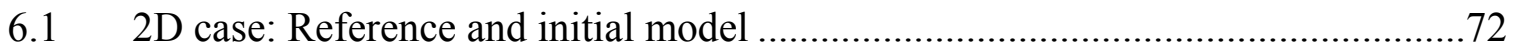

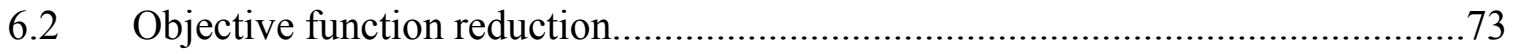

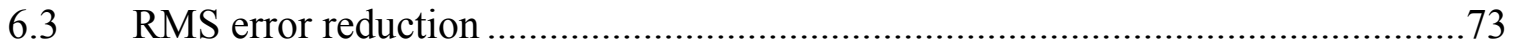

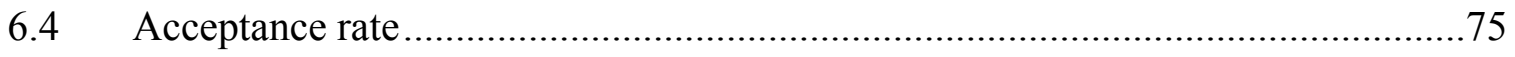

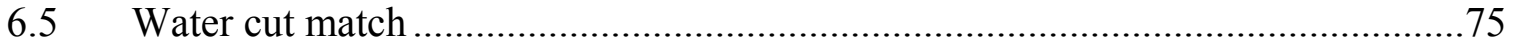

6.6 Convergence of parameters for approximate MCMC ….................................76

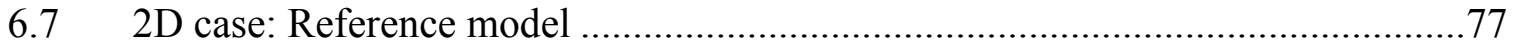

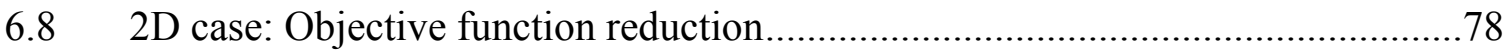


FIGURE

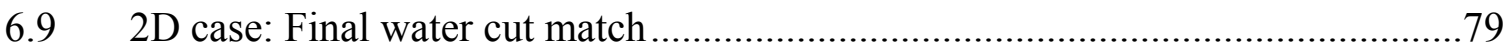

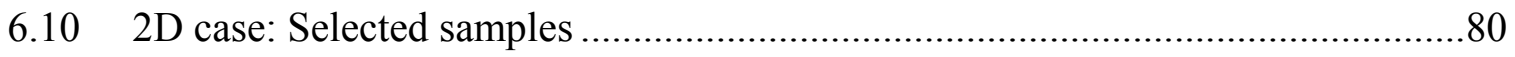

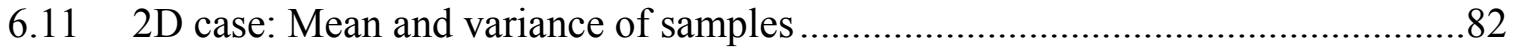

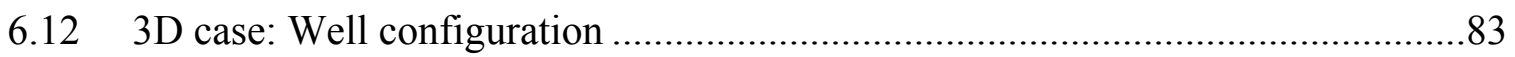

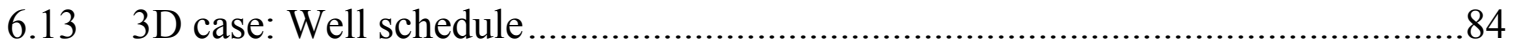

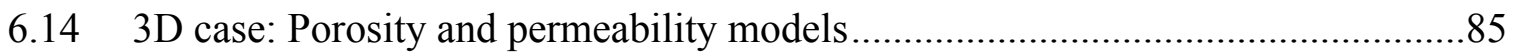

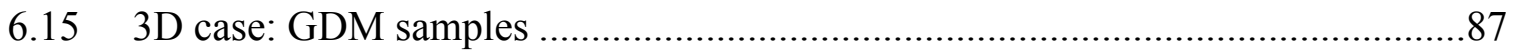

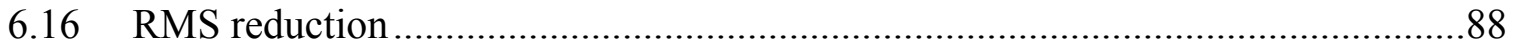

6.17 Acceptance rate for full and approximate MCMC .......................................89

$6.181^{\text {st }}$ sample: RMS reduction for full and approximate MCMC …........................90

6.19 Approximate MCMC: Three collected samples ............................................91

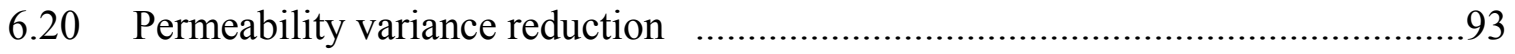

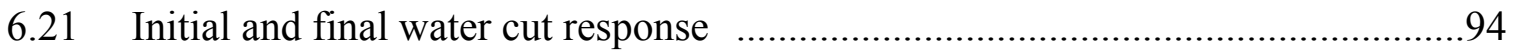

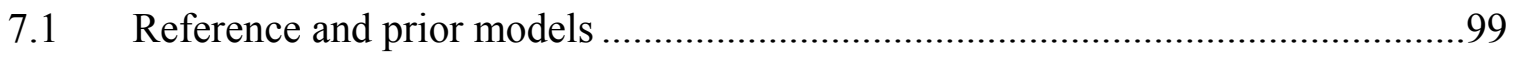

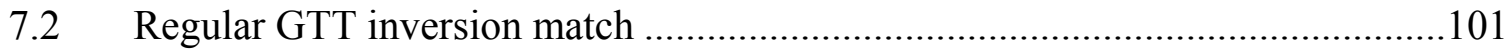

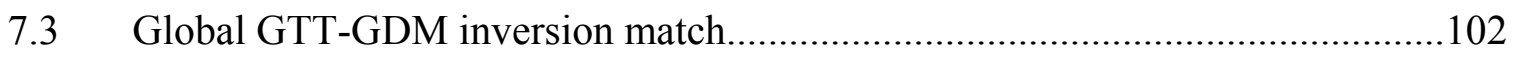

7.4 Local GTT-GDM inversion match using four local regions .............................103

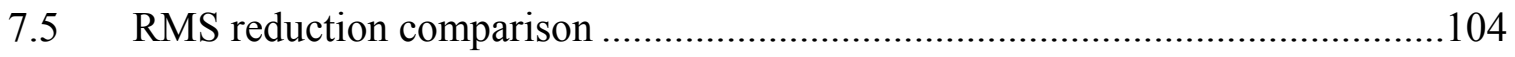




\section{CHAPTER I \\ INTRODUCTION}

\subsection{Literature Review}

Natural fractures are known to play a significant role in subsurface flow and transport of fluids. In recent years, advances in key technologies such as seismic imaging and horizontal drilling revealed the true extent of fractures in many reservoirs and enabled operators to utilize novel ways to use fracture connectivity to enhance recovery. The number of reservoirs that are now considered to be naturally fractured has also risen significantly in recent years and there is a greater need for more robust fracture characterization methods that can integrate both static and dynamic data in an efficient manner. ${ }^{1}$

Of late, discrete fracture network (DFN) techniques have gained increasing attention in the oil industry. ${ }^{2,3}$ The DFN is based on mapping fracture planes in 3D space using statistical properties of fracture swarms, fracture network geometry and flow characteristics. The advantage of the DFN models is the ability to incorporate complex fracture patterns based on field data such as cores, well logs, borehole images, seismic data and geomechanics. Although the DFN models can reproduce very realistic fracture geometry, it is important to condition these models to dynamic data such as well test, tracer and production data to reproduce the flow behavior in the reservoir. Such conditioning is particularly important for fractured reservoirs because only a small fraction of the fractures in the DFN model might carry bulk of the fluid flow. ${ }^{4,5}$

Streamline models have shown great potential in integrating dynamic data into high resolution geologic models. ${ }^{6-10}$ A unique feature of streamline models has been the ability to efficiently compute the sensitivity of the production data to reservoir parameters such as porosity and permeability. These sensitivities are partial derivatives that quantify how

The dissertation follows the style and format of SPE Journal. 
the production response will be affected by changes in reservoir properties. Integrating dynamic data into reservoir models typically involve the solution of an inverse problem and the sensitivities play a key role here. In our previous works, we have utilized the streamline-based sensitivities in conjunction with a generalized travel time inversion method to efficiently integrate production data into geologic models. ${ }^{7}$ Our approach has been successfully applied to a large number of field cases including a giant middleeastern carbonate reservoir. ${ }^{8}$

Until recently, streamline models have been limited to single porosity systems and thus, were not suitable for modeling fluid flow in fractured reservoirs, particularly accounting for matrix-fracture interactions. A common way to model fluid flow in fractured reservoirs is through the dual media approach whereby the fracture and the matrix are treated as separate continua that are connected through a transfer function. ${ }^{11-13}$ The transfer functions that describe the exchange of fluids between the matrix and the fracture system can be easily implemented within the framework of the current single porosity streamline models. ${ }^{14,15}$ This allows us to utilize much of the techniques related to production data integration developed for single porosity streamline models. However, compared to the single porosity systems, the propagation of the saturation front in the fracture is retarded significantly because of the exchange of fluid with the matrix in dual porosity systems. These effects must be accounted for while computing the travel time sensitivities for saturation fronts. The streamline-derived sensitivities can also be applied in conjunction with dual porosity finite difference simulators and allow us to combine the efficiency of the streamline approach with the versatility of finite difference simulation. The streamlines can be obtained from the fluid fluxes that are readily available during finite-difference simulation. This significantly broadens the applicability of the streamline-based approach in terms of incorporating compressibility effects and complex physics. $^{16}$

Our inverse problem is highly nonlinear and ill-posed ${ }^{17}$ and depending on the prior information, we can obtain a set of non unique solutions that honor both the prior constraints and conditioning data within the limits of uncertainty. The uncertainty in reservoir parameters is translated into uncertainty in reservoir response forecast that needs to be addressed in economic and operational risk analysis. In order to assess the 
uncertainty in estimated reservoir parameters we need to sample the parameters posterior distribution. The Bayesian formulation provides an excellent framework to perform this task. ${ }^{18}$ There are many methods proposed in literature. ${ }^{18}$ Generally, these methods either attempt to perform rigorous sampling of the posterior distribution, such as Markov Chain Monte Carlo (MCMC) methods and genetic algorithms ${ }^{19}$ or attempt to perform approximate sampling such as Randomized Maximum Likelihood (RML) ${ }^{17}$ and pilot point method. ${ }^{18}$ Rigorous methods, like MCMC, provide the most accurate sampling albeit at a high cost due to their high rejection rate and the need to run a full simulation for every proposed state. There is also a burn-in time needed for MCMC to assure that the starting state does not bias sampling which add to the cost. To avoid the high cost associated with rigorous methods, Oliver et $a l^{17}$ proposed a two step method with a high acceptance probability in the Metropolis-Hasting algorithm. Their method accomplishes this by first proposing an unconditional realization of the reservoir parameters and then history matching this unconditional realization using a perturbed production response obtained from adding noise constrained to the data covariance matrix. Due to the high acceptance rate of $95 \%$, they suggested accepting all the proposed new state in the chain. $^{17}$

Fox and Nicholls ${ }^{20}$ proposed the use of MCMC with an approximate likelihood to calculate the acceptance probability for the Metropolis-Hasting algorithm. According to their method, if the new state is accepted then the exact likelihood of the proposed state is calculated and the algorithm proceeds according to Metropolis-Hasting. If the new state is rejected during the approximate MCMC, then a new state is proposed and the algorithm is iterated. The obvious advantage here is that for high rejection rate algorithms like the traditional MCMC, The cost of calculating the exact likelihood, which in reservoir parameter uncertainty studies mean a full simulation run, is substantially reduced. The cost of the approximate solution is orders of magnitude less than the exact solution which translates into substantial savings in large scale reservoir models. In our work, the proposed state likelihood is approximated using semi-analytical streamlinebased sensitivities and production response, both obtained from a single simulation run using the initial state. Our method retains the rigorous sampling of traditional MCMC while substantially reducing the high cost associated with it. 


\subsection{Objectives}

The main objective of this work is to extend proven single porosity inversion methods to dual porosity reservoirs. In addition, more efficient perturbation methods and uncertainty assessment are formulated and explored using the gradual deformation method (GDM) and approximate MCMC approach. The specific objectives are summarized as follows:

- Derive a streamline-based analytical sensitivity method for dual porosity reservoirs.

- Compare analytical sensitivities with perturbation derived sensitivity coefficients.

- Incorporate the dual porosity streamline foreword model into the existing single porosity inversion algorithm.

- Test the dual porosity inversion algorithm using both 2D and 3D models

Reservoir models calibrated to dynamic data are not unique and carry uncertainty that need to be quantified for proper reservoir forecast assessment. To quantify uncertainty in reservoir parameters, we propose a two-stage MCMC that overcomes most of the traditional MCMC limitations and provide a fast and efficient parameter uncertainty assessment. The specific objectives of the proposed approach are as follows:

- Derive a locally linearized approximation to the flow simulation to calculate an approximate likelihood function using streamline-based analytical sensitivities.

- Generate geologically realistic proposals using global perturbation with the Gradual Deformation Method (GDM).

- Compare the proposed method performance against traditional MCMC.

- Test the proposed method using both synthetic 2D case and an actual 3D field case using Goldsmith dataset.

To handle large models with large number of data points, the re-parameterization of the inverse problem using an improved gradual deformation framework will be adopted. The following steps will be followed: 
- Derive a streamline-based analytical sensitivity for the general GDM formulation.

- Improve the optimization workflow to increase sampling efficiency.

- Write the algorithm code to perform the GDM with Generalized Travel Time (GTT) inversion and analytical sensitivity coefficients for both global and localized GDM.

- Test the algorithm using synthetic case and compare with the existing gradient based inversion method.

\subsection{Dissertation Outline}

Chapter II discusses the approach followed in integrating dynamic data in naturally fractured reservoirs using dual porosity single permeability (DPSP) formulation and generalized travel time inversion. Integrating dynamic data into high resolution fractured reservoirs involves the solution of an inverse problem which is computationally demanding. Solving the problem efficiently and reducing computational cost can be achieved by utilizing streamline-based sensitivities and the generalized travel time concept. Streamline and finite difference foreword models can be both used in our workflow which makes the approach applicable to a wide range of field conditions.

In Chapter III we discuss the mathematical formulation used in our approach. Dual porosity streamline-based analytical sensitivities is derived and compared to exact sensitivities obtained from numerical perturbation. We derived a general expression to account for cases where changing field conditions like infill drilling and rate fluctuations affect streamline distribution which is an extension to the robust single porosity formulation.

Chapter IV demonstrates our methodology using a 2D 9 spot water flood and a large $3 \mathrm{D}$ field with changing field conditions. In both cases we achieved a reasonable water cut match which shows the robustness of the approach under diverse field conditions.

In Chapter $\mathrm{V}$ we discuss the problem of uncertainty in reservoir parameters. The solution of the inverse problem is not unique and other plausible solutions exist. Risk analysis studies require a quantification of such uncertainty which can be 
computationally intractable for high resolution models. We proposed in this work a new method that substantially reduces the cost of sampling from reservoir parameter posterior distribution. The new method utilizes approximate likelihood to calculate MetropolisHasting acceptance criteria which is extremely efficient and requires a matrix multiplication instead of running a full simulation run. By filtering out obvious rejections without the need to run the forward model, the method maintains a high acceptance rate compared to traditional MCMC leading to substantial cost reductions. The method shares the traditional MCMC robustness since every accepted proposal is validated by calculating the exact likelihood before promoting the sample from initial to proposed state.

In Chapter VI we demonstrated the approximate MCMC method using both a $21 \times 21$ 2D 9-spot and a large 3D field case with a mesh size of $58 \times 53 \times 10$ or 30,740 grid cells. We compared the proposed method performance to traditional MCMC and demonstrated the substantial cost savings achieved.

In Chapter VII we discuss a different approach to dynamic data integration where geological structure is preserved using the gradual deformation method (GDM). In traditional GDM inversion, the number of deformed realizations is limited to two or three models due to the high cost of calculating numerical sensitivities. The limited number of realizations severely restricts the search direction and the use of GDM chains as a work around leads to even more inefficient workflow. We have derived streamline-based analytical sensitivities for GDM parameters that require a single simulation run regardless of the number of GDM parameters. The new method eliminates the need for GDM chains and maximizes the search direction by using a large number of basis models. We compared the new method to regular GTT inversion using both global and local GDM and showed that our method succeeds in achieving a match as good as the regular GTT while preserving the geologic structure. 


\section{CHAPTER II \\ APPROACH TO PARAMETER ESTIMATION IN FRACTURED MEDIUM}

Our approach for integrating dynamic data in fractured reservoirs and estimating fracture parameters is based on the concept of generalized travel time inversion for production data integration. ${ }^{21}$ The approach has been shown to be computationally efficient, robust and suitable for large-scale field applications. ${ }^{7,22}$ The unique aspect here is the extension and validation of streamline-based analytic travel time sensitivity computations for fractured medium and accounting for matrix-fracture exchange mechanisms. The travel time sensitivities can be applied to both streamline and finite difference simulators. Thus, we can exploit the computational efficiency of the streamline approach and the versatility of the finite difference simulators in terms of handling compressibility and complex physics. This chapter will discuss the general approach followed in integrating dynamic data in fractured medium.

\subsection{Dual Porosity Fracture Flow Simulation}

For modeling fluid flow in fractured reservoirs, we can use either a 3D dual porosity streamline simulator or a finite difference simulator. The streamline approach has

recently been extended to fractured reservoirs using the dual media approach. ${ }^{14,15}$ The streamline simulation uses an IMPES approach where fracture pressure is solved first then fracture saturation. Matrix saturation is updated using a transfer function that governs fluid exchange between the two mediums. The streamline approach decouples the transport calculation from the underlying heterogeneity thus simplifying the calculation. The solution is performed on the time of flight coordinate (TOF) and allows for larger time steps with fewer pressure updates without suffering from dispersion or numerical instability. When the underlying conditions are favorable, streamline 
simulation can be orders of magnitude faster than conventional finite difference simulators. In particular, the dual porosity streamline models can be considerably faster than conventional finite-difference simulators when the primary exchange mechanism between the matrix and the fracture system is capillary imbibition. The next chapter will cover the theory behind dual porosity streamline simulation in more depth.

In cases where strong coupling between the matrix and the fracture system exist, the streamline models may not offer significant advantage and we can revert to conventional finite difference dual porosity flow simulation. The use of finite-difference models allows us to incorporate compressibility and other relevant physical mechanisms without any significant loss in computational efficiency.

\subsection{Efficient Generalized Travel-Time Inversion}

The misfit between the observed and computed production response is quantified using a previously proposed generalized travel time. ${ }^{7,21}$ In the generalized travel time approach, which is an average travel time match, we seek to find the best overall match between observed and calculated response by systematically shifting the response on the time scale. The travel time approach is more robust and efficient than the traditional amplitude approach where we try to match the data directly. There are several advantages to using the generalized travel time approach for integrating dynamic data. Firstly, generalized travel time has quasi-linear convergence properties leading to fast

convergence and even if the prior model is not close to the solution. ${ }^{10}$ Secondly, the number of travel time matching data is reduced to the number of wells regardless of the number of data points per well leading to a more computationally efficient algorithm and less storage requirements. Finally, the generalized travel time is very effective in resolving large scale features in the reservoir.

\subsection{Sensitivity Computations}

A critical aspect of production data integration is calculation of sensitivities that define the relationship between production response and reservoir parameters. The 
sensitivity simply quantifies the change in reservoir response when a given reservoir parameter is perturbed which is a critical input to gradient based minimization methods. A fast and efficient way to calculate the sensitivities is vital to integrating dynamic data in high resolution models. The analytical sensitivities we derived for dual porosity medium are highly efficient in terms of computation and memory storage. We compute these sensitivities analytically as one-dimensional integrals along streamline trajectories in the fracture network. For dual porosity streamline simulators, these trajectories are readily available and only a single simulation run is needed to calculate both data misfit and sensitivities. However, for finite difference models an additional step is necessary to compute the streamlines and time of flight based on the finite difference velocity field at specific time steps. These one dimensional calculations scale very favorably with respect to number of grid blocks. Thus, our approach is particularly well-suited for high resolution geologic models.

\subsection{Data Integration and Objective Function Regularization}

Our objective is to incorporate dynamic data into high resolution fracture models. As we have mentioned earlier, we have used a generalized travel time inversion approach that utilizes analytical sensitivities in conjunction with an iterative optimization scheme to minimize the travel time shift between calculated and observed data. We start with a geologic model that already integrates well logs, seismic and geologic data using geostatistical modeling or other methods. The process of inversion will reconcile the high resolution model with observed field response. In order to achieve this, we minimize a locally linearized and panelized data misfit function. ${ }^{6,19}$

$$
F_{\text {Obj }}=\|\delta \mathbf{d}-\mathbf{S} \delta \mathbf{R}\|+\beta_{1}\|\delta \mathbf{R}\|+\beta_{2}\|\mathbf{L} \delta \mathbf{R}\|
$$

where $\delta \mathbf{d}$ is data misfit, $\delta \mathbf{R}$ is the change in reservoir parameter, $\mathbf{S}$ is the sensitivity matrix and $\mathbf{L}$ is a second-order spatial difference operator. The weights $\beta_{1}$ and $\beta_{2}$ determine the relative strengths of prior information and model roughness, respectively. The spatial operator is given by 


$$
\|\mathbf{L} \delta \mathbf{R}\|=\sum_{j=1}^{M}\left(\Delta \delta \mathbf{R}_{j}\right)^{2}
$$

The roughness constraint penalizes for high frequency spatial fluctuations in reservoir parameters since inversion is more effective in reproducing large scale features. This is mainly due to the fact that the observed data is the integrated response of all reservoir parameters. The norm constraints is given by

$$
\|\delta \mathbf{R}\|=\sum_{j=1}^{M}\left(\delta \mathbf{R}_{j}\right)^{2}
$$

and it penalizes large deviations from the prior model which already incorporates available static data. Minimizing deviation from the prior model insures that static data are also honored in the final model.

As discussed earlier, this formulation has many favorable characteristics including quasi-linear properties that make it attractive for field applications. ${ }^{7,15}$ 


\section{CHAPTER III \\ MATHEMATICAL FORMULATION}

In this chapter we will cover the mathematical formulation adopted in integrating dynamic data in dual porosity reservoirs using the generalized travel time inversion approach. We will first cover the theory of dual porosity streamline simulation and the concept of travel time and generalized travel time inversion. Then we will develop the dual porosity streamline-based analytical sensitivity using the generalized travel time framework and account for changing field conditions. Finally, we will verify the derived dual porosity sensitivity by comparing it to the exact numerical perturbation method.

\subsection{Dual Porosity Streamline Simulation}

Streamline models have recently been generalized to model fluid flow in fractured reservoirs including matrix-fracture interactions. ${ }^{14,15}$ A common approach to include such interactions has been through the dual porosity conceptualization whereby the fluid flow is assumed to occur primarily through the high permeability fracture system and the matrix acts as the fluid storage as demonstrated in Figure 3.1. ${ }^{11-13}$ 


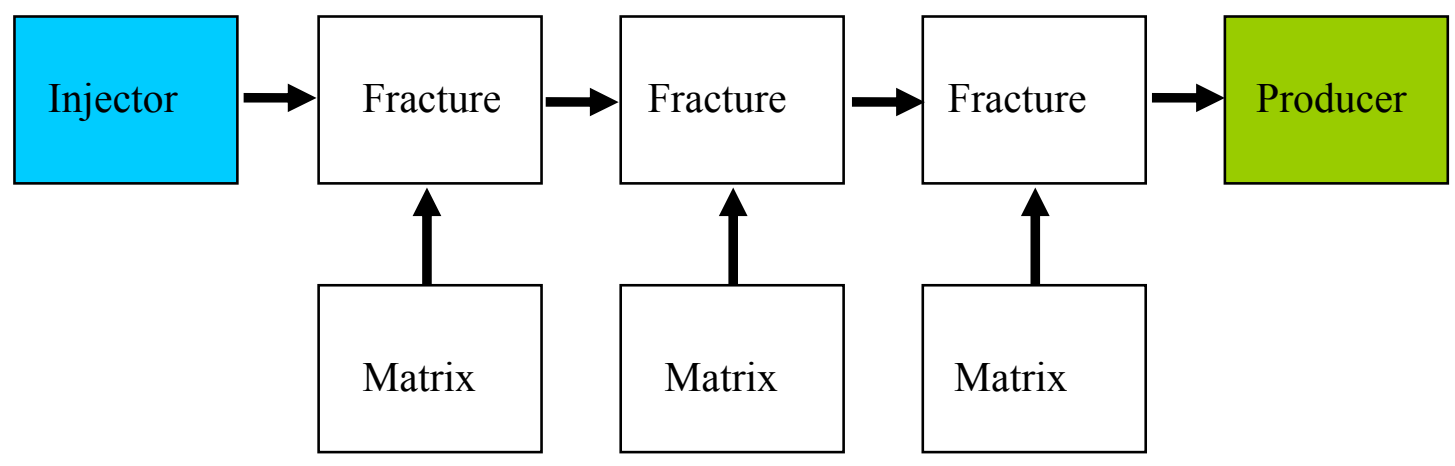

Figure 3.1 - Dual-porosity single-permeability (DPSP) system

A matrix-fracture transfer function is used to model the exchange of fluids between the matrix and the fracture systems.

\subsubsection{Pressure Equations}

For a dual porosity dual permeability incompressible two phase flow, the transport equations that govern fluid flow in fracture and matrix are given by the following. ${ }^{12,13}$

$$
\begin{aligned}
& \nabla \cdot k_{f} \cdot\left(\lambda_{o f} \nabla P_{o f}+\lambda_{o g f} \nabla Z_{f}\right)+\Gamma_{o}+q_{o f}=\phi \frac{\partial S_{o f}}{\partial t} \\
& \nabla \cdot k_{f} \cdot\left(\lambda_{w f} \nabla P_{w f}+\lambda_{w g f} \nabla Z_{f}\right)+\Gamma_{w}+q_{w f}=\phi \frac{\partial S_{w f}}{\partial t} \\
& \nabla \cdot k_{m} \cdot\left(\lambda_{o m} \nabla P_{o m}+\lambda_{o g m} \nabla Z_{f}\right)-\Gamma_{o}+q_{o m}=\phi \frac{\partial S_{o m}}{\partial t} \\
& \nabla \cdot k_{m} \cdot\left(\lambda_{w m} \nabla P_{w m}+\lambda_{w g m} \nabla Z_{m}\right)-\Gamma_{w}+q_{w m}=\phi \frac{\partial S_{w m}}{\partial t}
\end{aligned}
$$

$\lambda_{o g}$ and $\lambda_{w g}$ are the gravity terms given by 


$$
\begin{aligned}
& \lambda_{o g}=\frac{k_{r o} \rho_{o} g}{\mu_{o}} \\
& \lambda_{w g}=\frac{k_{r w} \rho_{w} g}{\mu_{w}}
\end{aligned}
$$

and $\Gamma_{o}$ and $\Gamma_{w}$ are the volumetric oil and water rates exchanged between the matrix and fracture systems. If we neglect capillarity effects and combine the equations for each system, we arrive at the fracture and matrix pressure equations

$$
\begin{aligned}
& \nabla \cdot k_{f} \cdot\left(\lambda_{t f} \nabla P_{f}+\lambda_{g f} \nabla Z_{f}\right)+\Gamma_{t}=-q_{s f} \\
& \nabla \cdot k_{m} \cdot\left(\lambda_{t m} \nabla P_{m}+\lambda_{g m} \nabla Z_{m}\right)-\Gamma_{t}=-q_{s m}
\end{aligned}
$$

where the combined terms are given by the following

$$
\begin{aligned}
& \lambda_{t}=\lambda_{o}+\lambda_{w} \\
& \lambda_{g}=\lambda_{o g}+\lambda_{w g} \\
& \Gamma_{t}=\Gamma_{o}+\Gamma_{w} .
\end{aligned}
$$

For dual porosity system where we have no flow between matrix blocks and no source term, Eq. 3.5 reduces to

$$
\Gamma_{t}=0
$$

We can conclude from Eq. 3.7 and 3.8 that matrix and fracture transfer terms are equal and magnitude and opposite in direction

$$
\Gamma_{o}=-\Gamma_{w}
$$


Since in dual porosity systems we have no flow between matrix blocks, streamlines will only be traced in the fracture network and only the fracture pressure equation is used. We can combine Eqs. 3.4 and 3.8 to arrive at the governing pressure equation for a dual porosity system.

$$
\nabla \cdot k_{f} \cdot\left(\lambda_{t f} \nabla P_{f}+\lambda_{g f} \nabla Z_{f}\right)=-q_{s f}
$$

Since the transfer term does not exist in the pressure equation, it will not affect streamlines trajectories. Eq 3.10 is used to solve for the velocity field which is used to trace streamlines.

\subsubsection{Saturation Equations}

If we consider incompressible flow in a non-deformable media, then the conservation equations for the fracture and the matrix in a dual porosity system can be written as follows, ${ }^{12-15}$

$$
\begin{aligned}
& \phi_{f} \frac{\partial S_{w f}}{\partial t}+\vec{u}_{t} \bullet \nabla f_{w f}+\nabla \bullet \vec{G}+\Gamma_{w}=0 \\
& \Gamma_{w}=\phi_{m} \frac{\partial S_{w m}}{\partial t}
\end{aligned}
$$

In Eqs. 3.11 and 3.12, the subscripts $f$ and $m$ represent the fracture and the matrix systems, respectively. In addition, the fractional flow, $f_{w f}$, and the gravity term, $G$, are defined as follows,

$$
f_{w f}=\frac{\lambda_{w f}}{\lambda_{w f}+\lambda_{o f}}
$$




$$
\vec{G}=k \cdot \frac{\lambda_{w f} \lambda_{o f}}{\lambda_{t}}\left(\rho_{o}-\rho_{w}\right) \vec{g} \nabla D
$$

where,

$$
\begin{aligned}
& \lambda_{w f}=\frac{k_{r w f}}{\mu_{w}} \ldots \\
& \lambda_{\text {of }}=\frac{k_{r o f}}{\mu_{o}} \ldots \\
& \lambda_{t}=\lambda_{\text {of }}+\lambda_{w f}
\end{aligned}
$$

In order to solve for saturation advancement along streamlines, we need to transform Eq. 3.11 into time of flight coordinates. For 3D irrotational potential flow and curl $\mathbf{u}=0$, we have the following relationship

$$
\mathbf{u}=\nabla \psi \times \nabla \chi
$$

where $\mathbf{u}$ is velocity, $\psi$ and $\chi$ are stream functions of flow in 3D space. Streamlines are defined as the intersection of stream surfaces in $\tau, \psi, \chi$ space. To calculate saturation advancement in time of flight coordinates, we need to transform streamlines from (x,y,z) grid coordinates to $(\tau, \psi, \chi)$ coordinates $^{23}$. Starting with the transformation of gradient operator, $\nabla$, from $(\mathrm{x}, \mathrm{y}, \mathrm{z})$ coordinates to $(\tau, \psi, \chi)$ coordinates

$$
\nabla=\nabla \tau \frac{\partial}{\partial \tau}+\nabla \psi \frac{\partial}{\partial \psi}+\nabla \chi \frac{\partial}{\partial \chi}
$$

The definition of time of flight is given by an integral along a streamline ${ }^{24}$ 


$$
\tau(x, y, z)=\int_{0}^{(x, y, z)} \frac{\phi_{f}}{\|\mathbf{u}\|} d r
$$

Eq. 3.20 can be rewritten in differential form

$$
\frac{d \tau}{d r}=\frac{\phi_{f}}{\|\mathbf{u}\|}
$$

$\frac{d \tau}{d r}$ is the change of $\tau$ as a tracer moves a infinitesimal distance of $\|d r\|$ along a given streamline. Since $d r$ is a vector in $(\mathrm{x}, \mathrm{y}, \mathrm{z})$ space, we can use the chain rule to expand Eq. 3.21

$$
\begin{aligned}
& \frac{\partial \tau}{\partial x} \frac{\partial x}{\partial r}+\frac{\partial \tau}{\partial y} \frac{\partial y}{\partial r}+\frac{\partial \tau}{\partial z} \frac{\partial z}{\partial r}=\frac{\phi_{f}}{\|\mathbf{u}\|} \\
& \left(\frac{\partial x}{\partial r} \hat{i}+\frac{\partial y}{\partial r} \hat{j}+\frac{\partial z}{\partial r} \hat{k}\right) \cdot\left(\frac{\partial \tau}{\partial x} \hat{i}+\frac{\partial \tau}{\partial y} \hat{j}+\frac{\partial \tau}{\partial z} \hat{k}\right)=\frac{\phi_{f}}{\|\mathbf{u}\|}
\end{aligned}
$$

If we examine the left hand side of $\mathrm{Eq} \mathrm{3.23,} \mathrm{we} \mathrm{notice} \mathrm{that} \mathrm{the} \mathrm{first} \mathrm{part} \mathrm{is} \mathrm{a} \mathrm{unit}$ vector along the velocity vector, $\mathbf{u}$, while the second part is $\nabla \tau$. We can rewrite Eq. 3.23 now in a more simplified form

$$
\mathbf{u} \cdot \nabla \tau=\phi_{f}
$$

The dot product of Eq. 3.19 with the velocity vector, $\mathbf{u}$, gives the following

$$
\mathbf{u} \cdot \nabla=\mathbf{u} \cdot \nabla \tau \frac{\partial}{\partial \tau}+\mathbf{u} \cdot \nabla \psi \frac{\partial}{\partial \psi}+\mathbf{u} \cdot \nabla \chi \frac{\partial}{\partial \chi}
$$


Recognizing that velocity vector is orthogonal to stream functions gradient, Eq. 3.25 simplifies to the following form

$$
\mathbf{u} \cdot \nabla=\mathbf{u} \cdot \nabla \tau \frac{\partial}{\partial \tau}
$$

We can now combine Eqs. 3.24 and 3.26 to arrive at the coordinate transform operator

$$
\mathbf{u} \cdot \nabla=\phi_{f} \frac{\partial}{\partial \tau}
$$

Finally, using the transform operator given Eq. 3.27, we can transform Eq. 3.11 in terms of streamline time of flight coordinate to arrive at the saturation equation for the fracture system

$$
\frac{\partial S_{w f}}{\partial t}+\frac{\partial f_{w f}}{\partial \tau}+\frac{\nabla \bullet \vec{G}}{\phi_{f}}+\frac{\Gamma_{w}}{\phi_{f}}=0
$$

Eq. 3.28 together with the matrix saturation Eq. 3.12 describes the streamline transport equations for the dual porosity system.

There are many forms for the transfer function in literature. In this study, we are assuming a countercurrent imbibition mechanism where the amount of water imbibed into the matrix block is equal to the amount of oil expelled into fracture. The conventional transfer function that model this exchange mechanism is given by $^{25,26}$

$$
\Gamma_{w}=F_{s} k_{m} \frac{\lambda_{w f} \lambda_{o m}}{\lambda_{w f}+\lambda_{o m}}\left(P_{c m}-P_{c f}\right)
$$

where $F_{s}$ is the shape factor and for a rectangular matrix block with all sides exposed to imbibition the following relationship is used ${ }^{12}$ 


$$
F_{s}=4\left(\frac{1}{l_{x}^{2}}+\frac{1}{l_{y}^{2}}+\frac{1}{l_{z}^{2}}\right)
$$

If we combine Eq. 3.28 with Eqs. 3.29 and 3.12, we arrive at the saturation equations for fracture and matrix blocks

$$
\begin{gathered}
\frac{\partial S_{w f}}{\partial t}+\frac{\partial f_{w f}}{\partial \tau}+\frac{\nabla \cdot \vec{G}_{f}}{\phi_{f}}+\frac{F_{s} k_{m}}{\phi_{f}} \frac{\lambda_{w m f} \lambda_{o m} f}{\lambda_{w m f}+\lambda_{o m f}}\left(P_{c m}-P_{c f}\right)=0 \\
\phi_{m} \frac{\partial S_{w m}}{\partial t}-\frac{F_{s} k_{m}}{\phi_{f}} \frac{\lambda_{w m f} \lambda_{o m} f}{\lambda_{w m f}+\lambda_{o m f}}\left(P_{c m}-P_{c f}\right)=0 \ldots \ldots \ldots \ldots \ldots \ldots \ldots \ldots \ldots \ldots \ldots \ldots
\end{gathered}
$$

To account for gravity effect, we employ the operator splitting technique ${ }^{27}$ and split Eq. 3.31 into two parts, the first part is the convective term which account for viscous forces along streamlines and is given by

$$
\frac{\partial S_{w f}}{\partial t}+\frac{\partial f_{w f}}{\partial \tau}+\frac{F_{s} k_{m}}{\phi_{f}} \frac{\lambda_{w m f} \lambda_{o m} f}{\lambda_{w m f}+\lambda_{o m f}}\left(P_{c m}-P_{c f}\right)=0
$$

The second part of the equation accounts for gravity effects only

$$
\frac{\partial S_{w f}}{\partial t}+\frac{\nabla \cdot \vec{G}_{f}}{\phi_{f}}=0
$$

During simulation, the convective term in Eq. 3.33 is solved first for saturation which is then used as an initial condition in Eq. 3.34 to calculate the final saturation during each time step. Eqs. 3.12 and 3.33 can be solved explicitly using the following discretization ${ }^{15}$ 


$$
\begin{gathered}
S_{w f, i}^{n+1}-S_{w f, i}^{n}=-\Delta t\left\{\begin{array}{l}
\frac{f_{w f, i}^{n}-f_{w f, i-1}^{n}}{\Delta \tau}+ \\
\left(\frac{F_{s} k_{m}}{\phi_{f}}\right)_{i}\left(\frac{\lambda_{w m f} \lambda_{o m f}}{\lambda w m f+\lambda o m f}\right)_{i}^{n}\left(P_{c m}-P_{c f}\right)_{i}^{n}
\end{array}\right\} \\
S_{w m, i}^{n+1}-S_{w m, i}^{n}=-\Delta t\left\{\left(\frac{F_{s} k_{m}}{\phi_{m}}\right)_{i}\left(\frac{\lambda_{w m f} \lambda_{o m f}}{\lambda w m f+\lambda o m f}\right)_{i}^{n}\left(P_{c m}-P_{c f}\right)_{i}^{n}\right\}
\end{gathered}
$$

\subsubsection{Streamline Tracing Steps}

As mentioned earlier, the fluid flow occurs only in the fracture system in dual porosity systems and we need to trace streamlines only for the fractured medium. The tracing of streamlines for the dual porosity system is identical to that of the single porosity system. ${ }^{28}$ The basic steps can be summarized as follows: (1) Starting with the fracture permeability field (Figure 3.2a), source/sink configuration and boundary conditions, a pressure field is generated as in conventional finite-difference simulation (Figure 3.2b). (2) Next, the velocity distribution in the reservoir is obtained using Darcy's law and the streamlines are traced using the Pollock approach ${ }^{28}$ (Figure 3.2c). The time of flight or travel time along streamlines is also obtained at this stage and the isochrones represent the front propagation (Figure 3.2d). (3) The fracture saturation distribution is obtained by solving the 1-D saturation Eq. 3.31(without the gravity term) along each streamline as shown in Figure 3.3a. Gravity effects can be accounted for in the same manner as in single porosity streamline simulation by using operator splitting

techniques. ${ }^{27}$ Figure $3.3 \mathrm{~b}$ shows the saturation distribution along a streamline as a function of matrix-fracture transfer rate in Eq. 10. For $F_{s}=0$, there is no interaction with the matrix and the solution reverts back to the single porosity formulation. Clearly, the net effect of the matrix-fracture transfer function is to impede the water saturation front advancement in the fracture system. The matrix saturation equation is solved along the streamline at the same time and is shown in Figure 3.3c. (4) The matrix and fracture saturations are then mapped back onto the grid (Figure 3.3e and Figure 3.3f). Again, the 
rapid propagation of the saturation front in the fracture system in the absence of transfer to the matrix $\left(F_{s}=0\right)$ can be clearly seen in Figure 3.3d. (5) The streamlines may be updated to account for changing well conditions such as infill drilling, rate changes etc. As in single porosity simulation, fracture and matrix saturations are mapped from streamlines onto the grid before each update, followed by pressure solution, streamline generation and re-initialization.

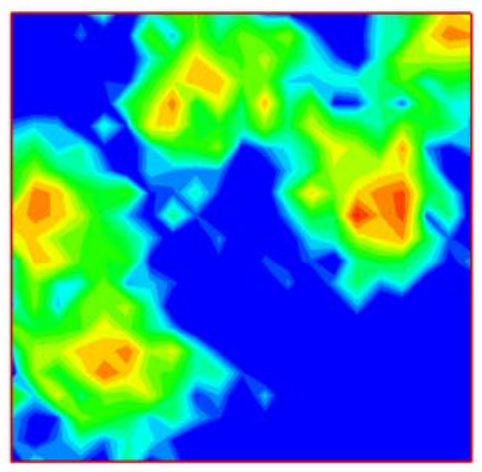

(a) Permeability field

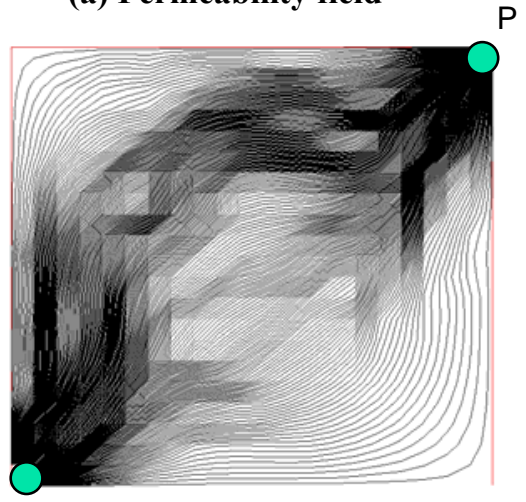

Inj (c) Streamlines

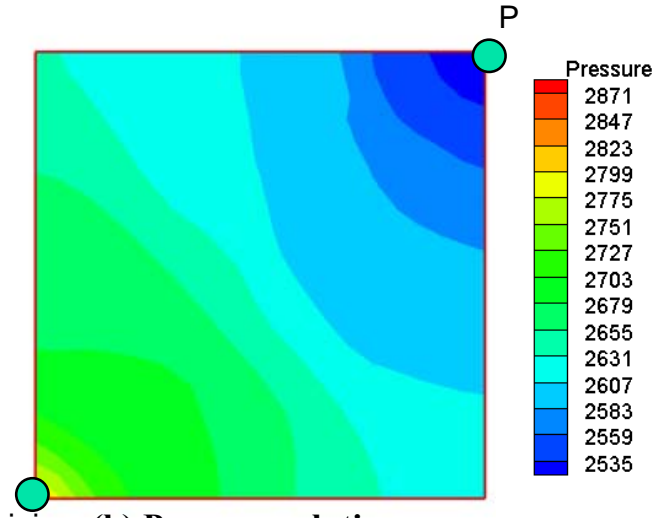

(b) Pressure solution

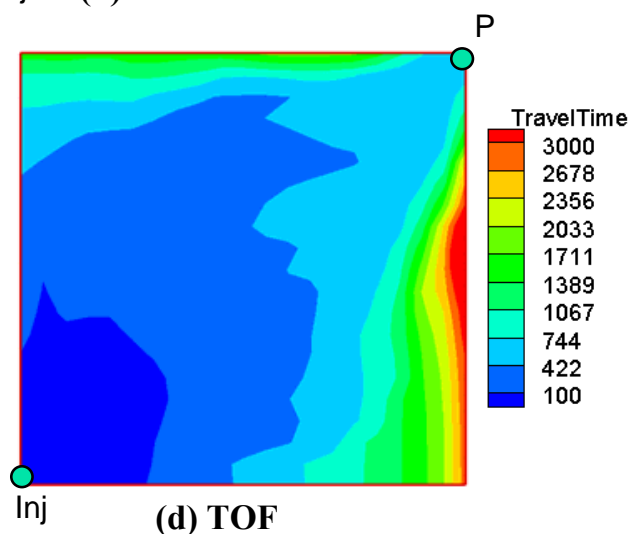

Figure 3.2 - Streamline and time of flight calculations 


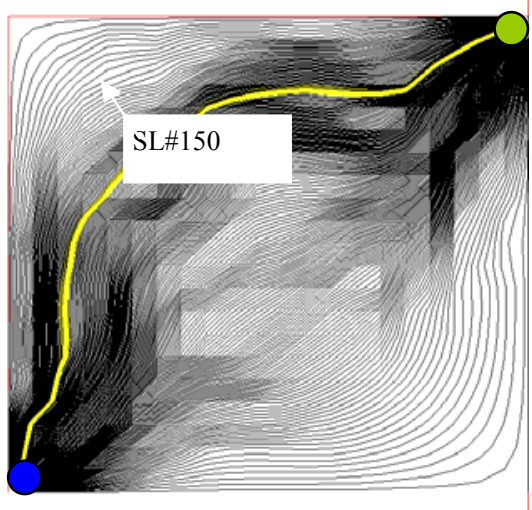

(a) 1-D streamlines

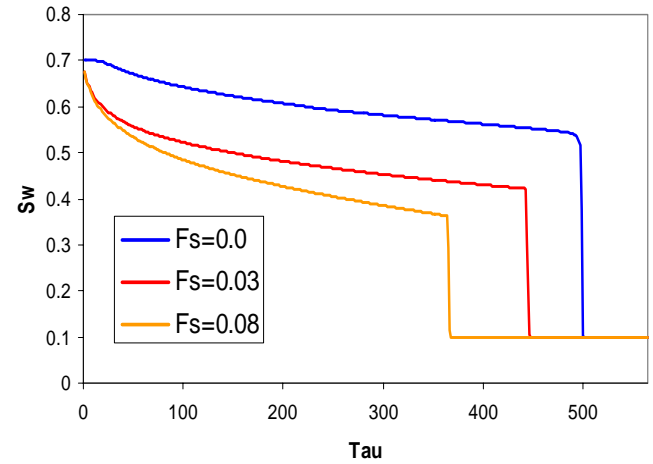

(b) Fracture saturation

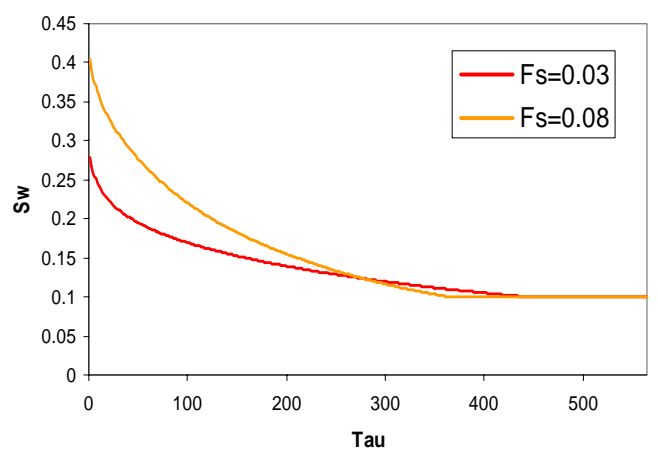

(c) Matrix

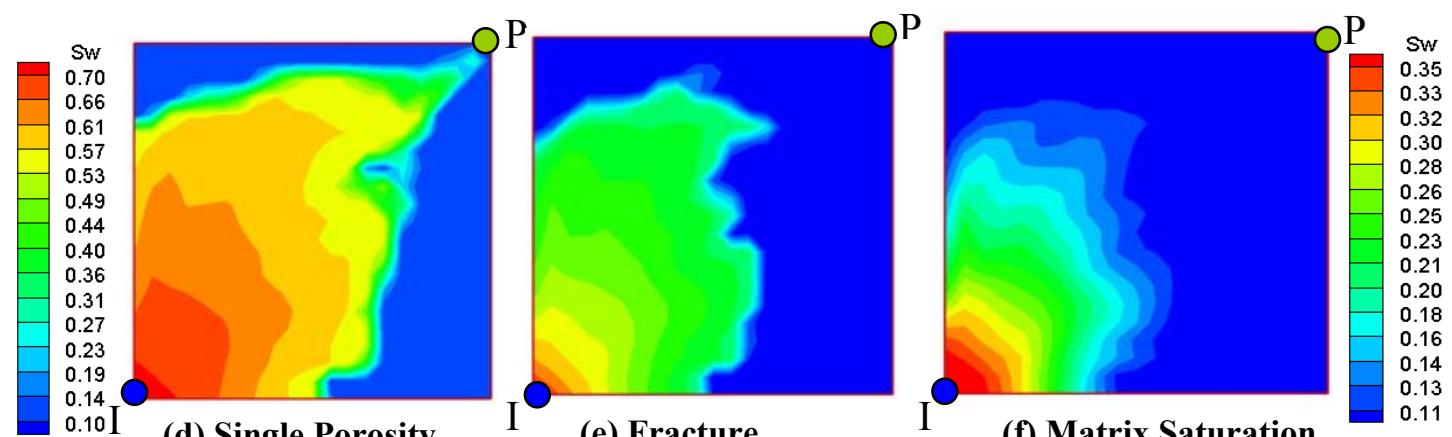

(d) Single Porosity

(e) Fracture

(f) Matrix Saturation

Figure 3.3 - Saturation evolution along streamlines - single and dual porosity examples 


\subsubsection{Pressure and Streamlines Updating}

In field applications, streamlines trajectories will be change over time due to changing field conditions ${ }^{7}$. Changes ranges from moderate in cases of production and injection rates fluctuations to the more drastic in cases of infill drilling which completely changes streamlines configuration in affected areas. Even if conditions are assumed constant, the evolution of saturation over time will alter total mobility which in turn will affect streamlines trajectories. To account for all of these changes, streamlines need to be updated whenever necessary by remapping saturations back onto the grid and updating the pressure and velocity fields in order to regenerate streamlines.

\subsection{Data Misfit Calculation}

The first step in integrating production data is to quantify of the data misfit. Data misfit is a measure of discrepancy between observed and calculated responses. In our approach, we define a 'generalized travel time' at each well for this purpose. We seek an optimal time-shift $\Delta t$ at each well so as to minimize the production data misfit at the well. ${ }^{21}$ This is illustrated in Figure 3.4a where the calculated water-cut response is systematically shifted in small time increments towards the observed response and the

data misfit is computed for each time increment. The optimal shift will be given by $\Delta t$ that minimizes the misfit function,

$$
J=\sum_{i=1}^{N d}\left[y^{o b s}\left(t_{i}+\Delta t\right)-y^{c a l}\left(t_{i}\right)\right]^{2}=f(\Delta t, m)
$$

or, alternatively maximizes the coefficient of determination given by the following

$$
R^{2}(\Delta t)=1-\frac{\sum\left[y^{o b s}\left(t_{i}+\Delta t\right)-y^{c a l}\left(t_{i}\right)\right]^{2}}{\sum\left[y^{o b s}\left(t_{i}\right)-\overline{y^{o b s}}\right]^{2}} .
$$




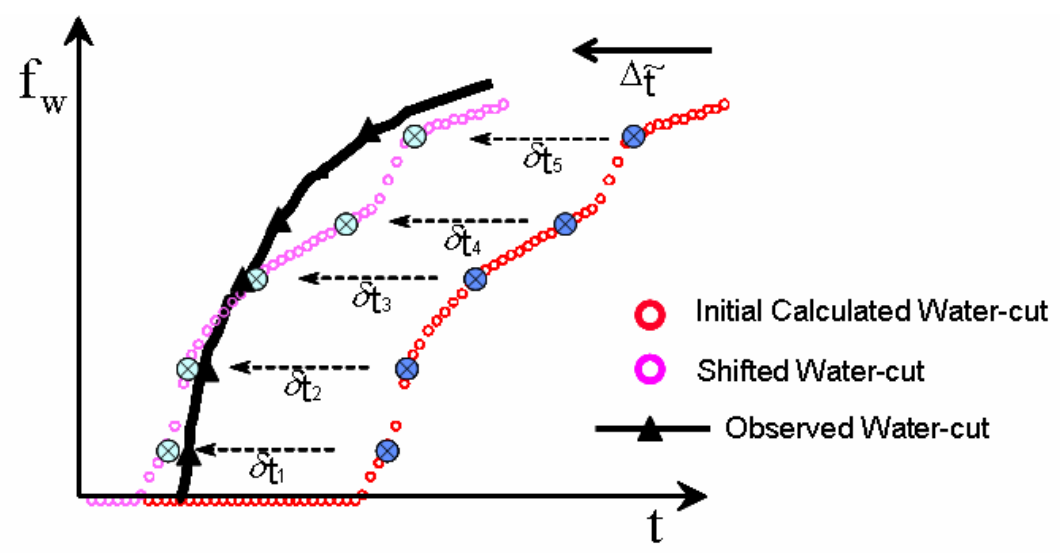

(a) Time shift

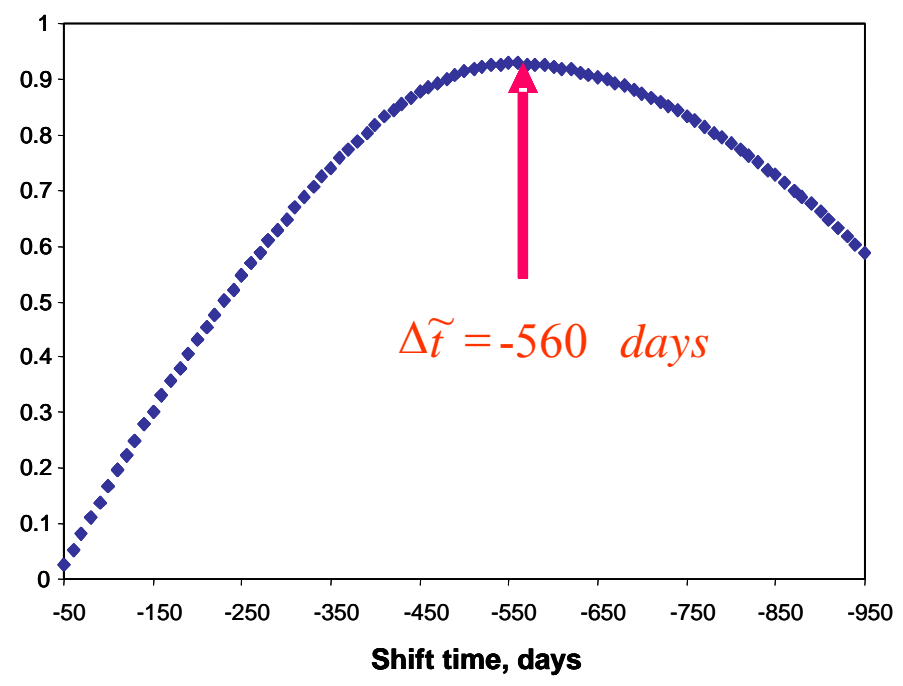

(b) Maximizing correlation

Figure 3.4 - Illustration of generalized travel-time shift

Thus, we define the generalized travel time as the 'optimal' time-shift $\Delta \tilde{t}$. that maximizes the $\mathrm{R}^{2}$ as shown in Figure $3.4 \mathrm{~b}$. It is important to point out that the computation of the optimal shift does not require any additional flow simulations. It is carried out as a post-processing at each well after the calculated production response is obtained from flow simulation. The overall production data misfit can now be expressed in terms of a generalized travel time misfit at all wells as follows 


$$
E=\sum_{j=1}^{N w}\left(\Delta \tilde{t}_{j}\right)^{2}
$$

The generalized travel time approach has been successfully applied to many field cases. It leads to a robust and efficient inversion scheme because of its quasi-linear properties. $^{7,21}$

\subsection{Sensitivity for Dual Porosity Systems}

One of the important advantages of the streamline approach is the ability to analytically compute the sensitivities of the generalized travel time with respect to reservoir parameters. Streamline-based sensitivities have made it feasible to integrate dynamic data into high resolution geological models ${ }^{6-8}$. Analytical sensitivities will form an integral part of our data integration approach. In this work, we will extend dynamic data integration methods to dual porosity systems by developing an expression for dual porosity analytical sensitivities.

We have seen that during generalized travel time computation we shift the entire fractional flow curve by a constant time. Thus, every data point in the fractional-flow curve has the same time shift, $\delta t_{1}=\delta t_{2}=\cdots=\Delta \tilde{t}$ (Figure 3.4a). We can average the travel time sensitivities of all data points to obtain a rather simple expression for the generalized travel time sensitivity with respect to fracture parameters $m$ as follows, ${ }^{7}$

$$
\frac{\partial \Delta \tilde{t}_{j}}{\partial m}=-\frac{\sum_{i=1}^{N_{d j}}\left(\partial t_{i, j} / \partial m\right)}{N_{d j}}
$$

All that remains now is to calculate the dual porosity travel time sensitivities, $\partial t_{i, j} / \partial m$, of various water-cut at the producing well. In the following sections we will derive and verify an analytical expression for travel time sensitivities in dual porosity systems. 


\subsubsection{Dual Porosity Analytical Sensitivities}

Perturbing fracture parameters will greatly affect the convective transport of fluids in the fracture network. We have seen earlier that Eq. 3.33 models the convective part of saturation evolution along streamlines in the fracture network. Using the operator splitting technique, we can split Eq. 3.33 into two parts: a predictor that models transport along streamlines and a corrector that accounts for matrix-fracture fluid exchange as follows

$$
\begin{aligned}
& \frac{\partial S_{w f}}{\partial t}+\frac{\partial f_{w f}}{\partial \tau}=0: \text { Predictor } \\
& \frac{\partial S_{w f}}{\partial t}+\frac{\Gamma_{w}}{\phi_{f}}=0: \text { Corrector }
\end{aligned}
$$

Perturbing fracture parameters will affect the transport along streamlines which is modeled by the predictor part. Rearranging Eq. 3.41 and using the chain rule,

$$
\frac{\partial S_{w f}}{\partial t}=-\frac{\partial f_{w f}}{\partial S_{w f}} \frac{\partial S_{w f}}{\partial \tau}
$$

If we assume that small perturbations to fracture parameters do not shift streamlines in space, then the change in saturation at the streamline outlet node is the sum of dynamic change and parameter perturbation effect,

$$
\delta S_{w f}=\frac{\partial S_{w f}}{\partial t} \delta t+\frac{\partial S_{w f}}{\partial \tau}\left[\frac{\partial \tau}{\partial \mathbf{m}}\right]^{T} \delta \mathbf{m}
$$

The propagation of a fixed saturation is found by setting $\delta S_{w f}=0$ 


$$
0=\frac{\partial S_{w f}}{\partial t} \delta t+\frac{\partial S_{w f}}{\partial \tau}\left[\frac{\partial \tau}{\partial \mathbf{m}}\right]^{T} \delta \mathbf{m}
$$

Combining Eqs. 3.43 and 3.45 and differentiating the expression with respect to a given reservoir parameter, $m$, we arrive at the following expression for the travel time sensitivity in terms of time of flight sensitivity

$$
\frac{\partial t}{\partial m}=\frac{\frac{\partial \tau}{\partial m}}{\frac{\partial f_{w f}}{\partial S_{w f}}} .
$$

It should be noted that the fractional flow derivative, $\frac{\partial f_{w f}}{\partial S_{w f}}$, is evaluated after saturation is updated using Eq. 3.42 to account for matrix-fracture fluid exchange. If gravity is included, then an additional updating is required to account for gravity segregation before the sensitivities are computed. ${ }^{27}$ The Time Of Flight (TOF) sensitivity is calculated from the definition of time of flight given in Eq. 3.20 which can be readily evaluated after a single simulation run. For example, the TOF sensitivity with respect to fracture permeability can be derived by substituting the Darcy velocity definition,

$$
\mathbf{u}=\lambda_{t} k_{f}(\mathbf{x})|\nabla P(\mathbf{x})|
$$

into Eq. 3.20

$$
\tau=\int_{\Sigma} \frac{\phi_{f}(\mathbf{x})}{\lambda_{t} k_{f}(\mathbf{x})|\nabla P(\mathbf{x})|} d x
$$

Here, $\sum$ stands for performing the integration over the length of a given streamline. Differentiating Eq. 3.48 with respect to fracture permeability gives the required 
sensitivity ( $\mathrm{x}$ accounts for the fact that the integration is one dimensional along a streamline),

$$
\frac{\partial \tau}{\partial k_{f}(\mathbf{x})}=\int_{\Sigma} \frac{\partial}{\partial k_{f}(\mathbf{x})}\left(\frac{\phi_{f}(\mathbf{x})}{\lambda_{t} k_{f}(\mathbf{x})|\nabla P(\mathbf{x})|}\right) d x=-\int_{\Sigma} \frac{\phi_{f}(\mathbf{x})}{\lambda_{t} k_{f}^{2}(\mathbf{x})|\nabla P(\mathbf{x})|} d x
$$

This integral can be evaluated as a summation over a given streamline,

$$
\frac{\partial \tau}{\partial k_{f}(\mathbf{x})}=-\sum \frac{\phi_{f}(\mathbf{x})}{\lambda_{t} k_{f}^{2}(\mathbf{x})|\nabla P(\mathbf{x})|} \Delta x
$$

Note that the quantities in the summation expression are either contained in the initial reservoir model or are available after the forward simulation run. The negative sign in Eq. 3.50 is consistent with the fact that increasing fracture permeability will reduce time of flight, hence giving a negative sensitivity.

\subsubsection{Verification of Dual Porosity Sensitivity}

In order to verify our DPSP travel time sensitivity in Eq. 3.46 we compared our results with sensitivities obtained by numerical perturbation. For this purpose, we simulated water injection in a quarter five-spot pattern. A dual porosity medium with homogeneous fracture permeability represented by $21 \times 21$ grid cells was used for this comparison. We perturbed every grid block permeability by $5 \%$, one grid block at a time and numerically computed the partial derivative of the arrival time of a fixed water cut with respect to permeability. Figure 3.5 shows the results for water cuts of 0.10 and 0.20 . Clearly, we obtain a good agreement between analytical travel time sensitivities calculated from Eq. 3.46 and numerical travel time sensitivities. The perturbation method shows some artifacts partly because the results depend on the magnitude of perturbation whereas the analytical sensitivities are symmetric and smooth. The differences are also because of the approximations inherent in the analytical computations, particularly the assumption that the streamlines do not shift because of small perturbation in reservoir 
properties. Nevertheless, as we will see later, the streamline-based sensitivities are adequate for history matching purposes under a wide variety of conditions.
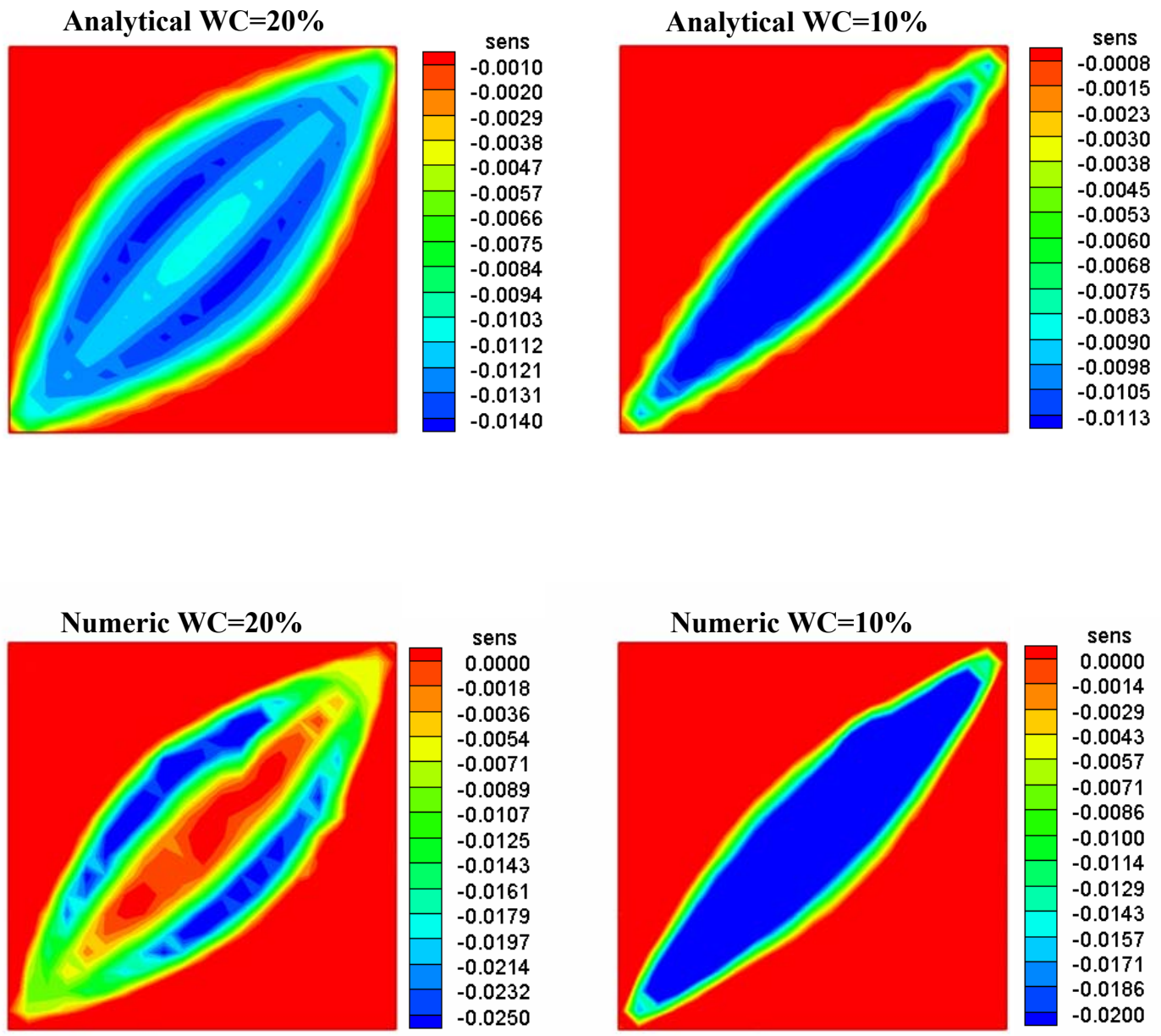

Figure 3.5 - Comparison of numerical and analytical sensitivities in

\section{a $1 / 4$-five spot pattern}




\subsubsection{Accounting for Changing Field Conditions}

The analytical sensitivities we have derived earlier does not account explicitly for saturation distributions along streamlines. During pressure updates, which account for changing field conditions, permeability perturbations will affect saturation distributions. We can account for such conditions using the same approach used in single porosity systems. ${ }^{7}$ Changes to the streamline outlet node saturation are now a function of dynamic changes, parameter perturbation and initial water saturation at the beginning of the pressure update cycle. Extending Eq. 3.44,

$$
\delta S_{w f}^{n+1}=\frac{\partial S_{w f}^{n+1}}{\partial t} \delta t+\frac{\partial S_{w f}^{n+1}}{\partial \tau}\left[\frac{\partial \tau}{\partial \mathbf{m}}\right]^{T} \delta \mathbf{m}+\left[\frac{\partial S_{w f}^{n+1}}{\partial \mathbf{S}_{w f}^{n}}\right]^{T} \delta \mathbf{S}_{w f}^{n}
$$

where $n$ and $n+1$ represent previous and current pressure updates, respectively. Here, $S_{w f}^{n}$ are the initial fracture saturations for the current pressure update along a streamline. The change to saturation at the outlet node is mainly a function of its own initial saturation rather than the initial saturations of other nodes. Rewriting Eq. 3.51,

$$
\delta S_{w f}^{n+1}=\frac{\partial S_{w f}^{n+1}}{\partial t} \delta t+\frac{\partial S_{w f}^{n+1}}{\partial \tau}\left[\frac{\partial \tau}{\partial \mathbf{m}}\right]^{T} \delta \mathbf{m}+\frac{\partial S_{w f}^{n+1}}{\partial S_{w f}^{n}} \delta S_{w f}^{n}
$$

It was found that this approximation has minimal impact on the sensitivity calculation ${ }^{7}$. Including the effect of reservoir parameter perturbation on initial saturation through the chain rule we get the following form,

$$
\delta S_{w f}^{n+1}=\frac{\partial S_{w f}^{n+1}}{\partial t} \delta t+\frac{\partial S_{w f}^{n+1}}{\partial \tau}\left[\frac{\partial \tau}{\partial \mathbf{m}}\right]^{T} \delta \mathbf{m}+\frac{\partial S_{w f}^{n+1}}{\partial S_{w f}^{n}}\left[\frac{\partial S_{w f}^{n}}{\partial \mathbf{m}}\right]^{T} \delta \mathbf{m}
$$

Using the same approach we did earlier, we set $\delta S_{w f}^{n+1}=0$ for a fixed saturation. 


$$
0=\frac{\partial S_{w f}^{n+1}}{\partial t} \delta t+\frac{\partial S_{w f}^{n+1}}{\partial \tau}\left[\frac{\partial \tau}{\partial \mathbf{m}}\right]^{T} \delta \mathbf{m}+\frac{\partial S_{w f}^{n+1}}{\partial S_{w f}^{n}}\left[\frac{\partial S_{w f}^{n}}{\partial \mathbf{m}}\right]^{T} \delta \mathbf{m}
$$

To find sensitivities, we differentiate Eq. 3.54 with respect to a given reservoir parameter, $m$, to get the following expression after rearrangement.

$$
\frac{\partial t}{\partial m}=\frac{\frac{\partial S_{w f}^{n+1}}{\partial \tau} \frac{\partial \tau}{\partial m}+\frac{\partial S_{w f}^{n+1}}{\partial S_{w f}^{n}} \frac{\partial S_{w f}^{n}}{\partial m}}{\frac{\partial S_{w f}^{n+1}}{\partial t}}
$$

Substituting Eq. 3.43 into the above expression gives us the sensitivity expression

$$
\frac{\partial t}{\partial m}=\frac{\frac{\partial \tau}{\partial m}}{\frac{\partial f_{w f}}{\partial S_{w f}}}+\left[\frac{\partial t}{\partial m}\right]^{n}
$$

where $\left[\frac{\partial t}{\partial m}\right]^{n}$ is the sensitivity at the end of the last pressure update cycle. For the first cycle or in the case of a single pressure update cycle, $\left[\frac{\partial t}{\partial m}\right]^{n}$ is set to zero. As the previous sensitivity expression, Eq. 3.56 requires only a single simulation run to be evaluated.

\subsection{Dynamic Data Integration}

Data inversion various approaches have been proposed in the literature for the integration of production data via inverse modeling. ${ }^{29-33}$ These can be broadly classified into 'deterministic' and 'Bayesian' methods. Both methods have been successfully applied to history matching of field data. In this work, we have adopted a Bayesian formulation whereby we minimize the following penalized misfit function, 


$$
\frac{1}{2}\left(m-m_{p}\right)^{T} C_{M}^{-1}\left(m-m_{p}\right)+\frac{1}{2}[\Delta \tilde{\mathbf{t}}]^{T} C_{D}^{-1}[\Delta \tilde{\mathbf{t}}]
$$

In Eq. $3.57, \Delta \tilde{\mathbf{t}}$ is the vector of generalized travel-time shift at the wells; $C_{D}$ and $C_{M}$ are the data error covariance and the prior model parameter covariance, respectively. The minimum in Eq. 3.57 can be obtained by an iterative least-squares solution to the linear system $^{34}$

$$
\left[\begin{array}{l}
\mathbf{C}_{D}^{-1 / 2} \mathbf{G} \\
\mathbf{C}_{\mathbf{M}}^{-1 / 2}
\end{array}\right] \delta \mathbf{m}=\left[\begin{array}{l}
\mathbf{C}_{D}^{-1 / 2}(\Delta \tilde{\mathbf{t}}) \\
\mathbf{C}_{\mathbf{M}}^{-1 / 2}\left(\mathbf{m}_{\mathbf{p}}-\mathbf{m}\right)
\end{array}\right]
$$

where $\mathbf{G}$ is the sensitivity matrix containing the sensitivities of the generalized travel time with respect to the reservoir parameters and $\mathbf{m}_{\mathbf{P}}$ represents the prior model. We use an iterative sparse matrix solver, LSQR, for solving the augmented linear system in Eq. 3.58. The LSQR algorithm is well suited for highly ill-conditioned systems and has been widely used for large-scale tomographic problems in seismology. ${ }^{35}$

An important consideration in the solution of Eq. 3.58 is calculation of the squareroot of the inverse of the prior covariance matrix. We have used a numerical stencil that allows for an extremely efficient computation of $C_{M}^{-1 / 2}$ and is applicable to a wide range of covariance and variogram models. ${ }^{36}$ 


\section{CHAPTER IV \\ APPLICATION OF DUAL POROSITY INVERSION}

In this chapter we will cover the application of dual porosity dynamic data integration using both $2 \mathrm{D}$ and $3 \mathrm{D}$ models. We will cover first the modeling part using discrete fracture network modeling where a realistic high resolution fractures models can be generated using field derived data.

\subsection{D 9-Spot Water Flood Pattern}

To illustrate our approach, we will use an example that involves integration of water cut data in a 9-spot pattern. We started with the discrete fracture network shown in Figure 4.1a. The model exhibits complex connectivity patterns common to naturally fractured reservoir where the distribution of fracture swarms determines the shape and intensity of fractured regions. A moving window is used to calculate the fracture density for each grid cell which is then converted to a fracture permeability multiplier using a non-linear transform. ${ }^{37}$ The fracture permeability is calculated using the multiplier and a predetermined fracture permeability range. We generated the permeability field show in Figure $4.1 \mathrm{~b}$ using a $21 \times 21$ grid. We used a dual porosity streamline simulator for modeling fluid flow in the fractured medium for this example. In Figure 4.2, which shows well locations and generated streamlines for the reference model, we can see how the streamlines are more concentrated in highly fractured regions to the South West of the model. Wells 1,2 and 4 are more connected to the injector than the rest of the producers which will lead to earlier breakthrough as we will see later.

We can randomly extract various percentages of fracture swarms and fractures within the swarms to generate prior models with varying degrees of fracture information. Because production data is more appropriate for characterizing large scale features, fracture swarms location is more critical than the detailed connectivity of individual 


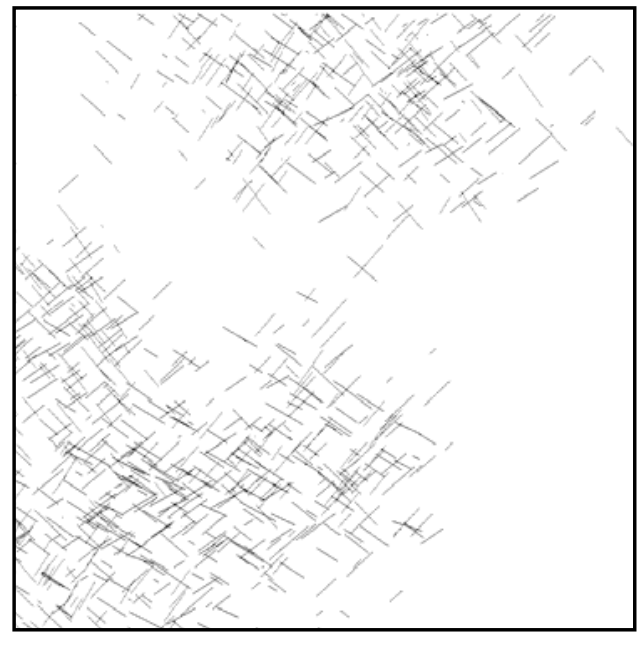

(a) Discrete fracture network

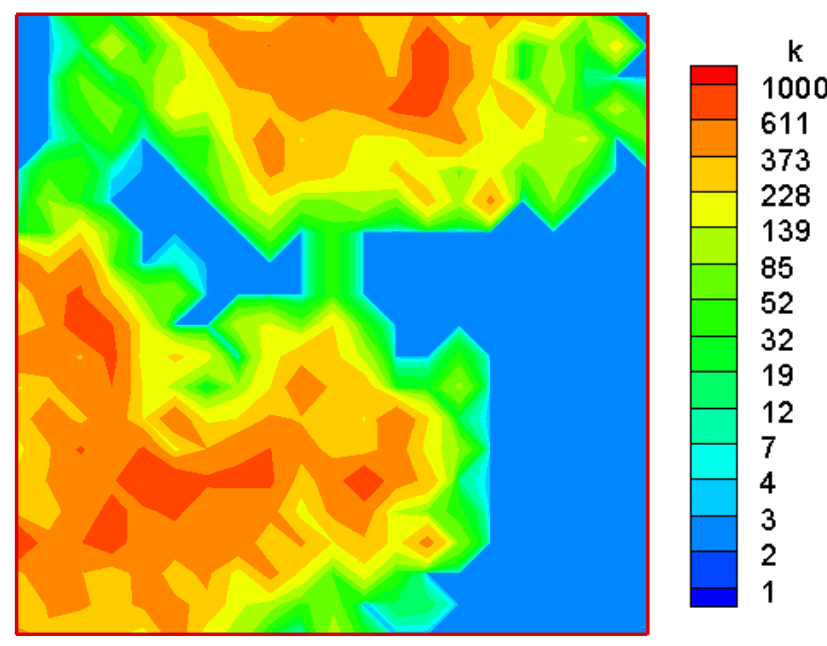

(b) Reference fracture permeability

Figure 4.1 - Reference model for the 9-spot 2D case

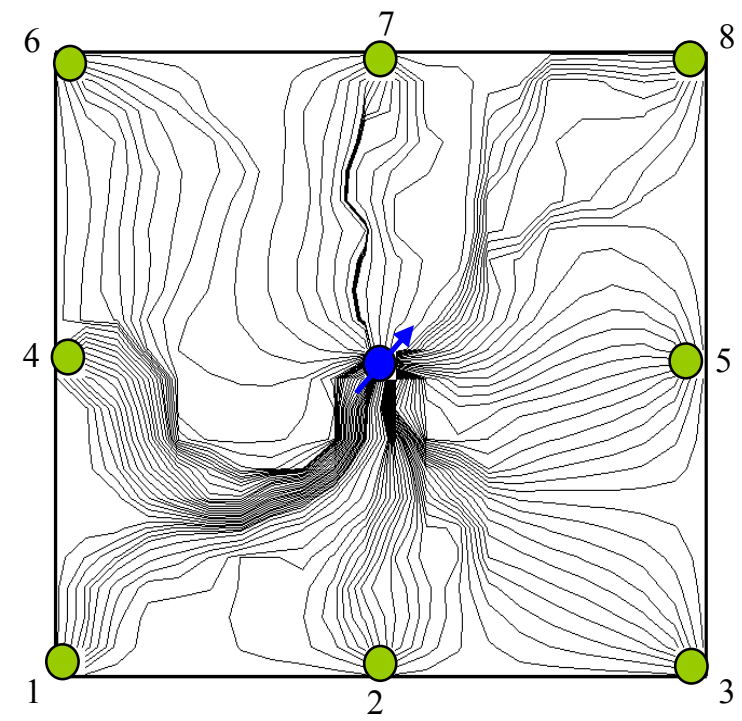

Figure 4.2 - Well locations and streamlines

fractures within a cell. To generate a 2D prior model of fracture patterns, we randomly draw $50 \%$ of the fracture swarms and $50 \%$ of fractures inside each swarm. Figure $4.3 \mathrm{a}$ shows the prior fracture permeability model.

We match the water cut response from the reference model for the first 500 days using the generalized travel time inversion. Starting with the prior model, we minimize the travel time shift at each producer iteratively to match the reference production data. 
Figure $4.3 \mathrm{~b}$ shows the final fracture permeability model. Figure 4.4 shows the observed data, initial model response and the matched response after performing the generalized travel time inversion.

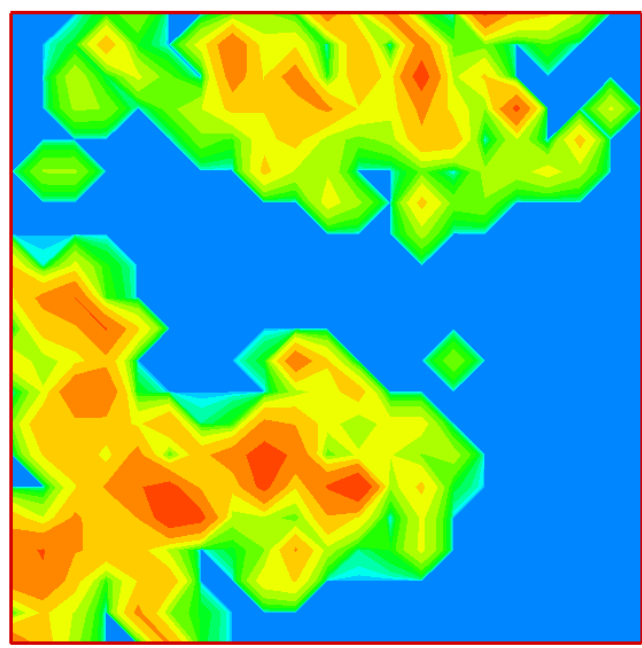

(a) Prior permeability model

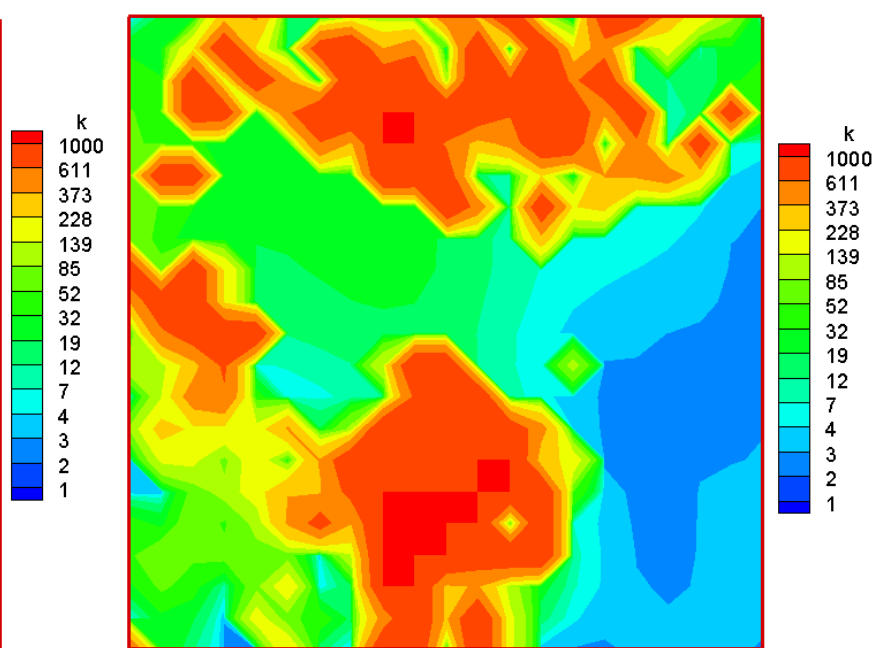

(b) Final match model

Figure 4.3 - Prior and match models

The process has not only matched the breakthrough times but also the amplitude of the water cut response for all the wells. Also, Figure 4.3b shows that after inversion we are able to recover the permeability contrast in the reference model and reproduce the dominant fracture connectivity while retaining most of the features of the prior model. For example, integration of production data has connected the two distinct high permeability regions in the prior model. This is clearly an important feature in the reference model in terms of fluid flow response. Finally, Figure 4.5 shows the convergence of the inversion algorithm. The data misfit is reduced by almost an order of magnitude in only five iterations. 


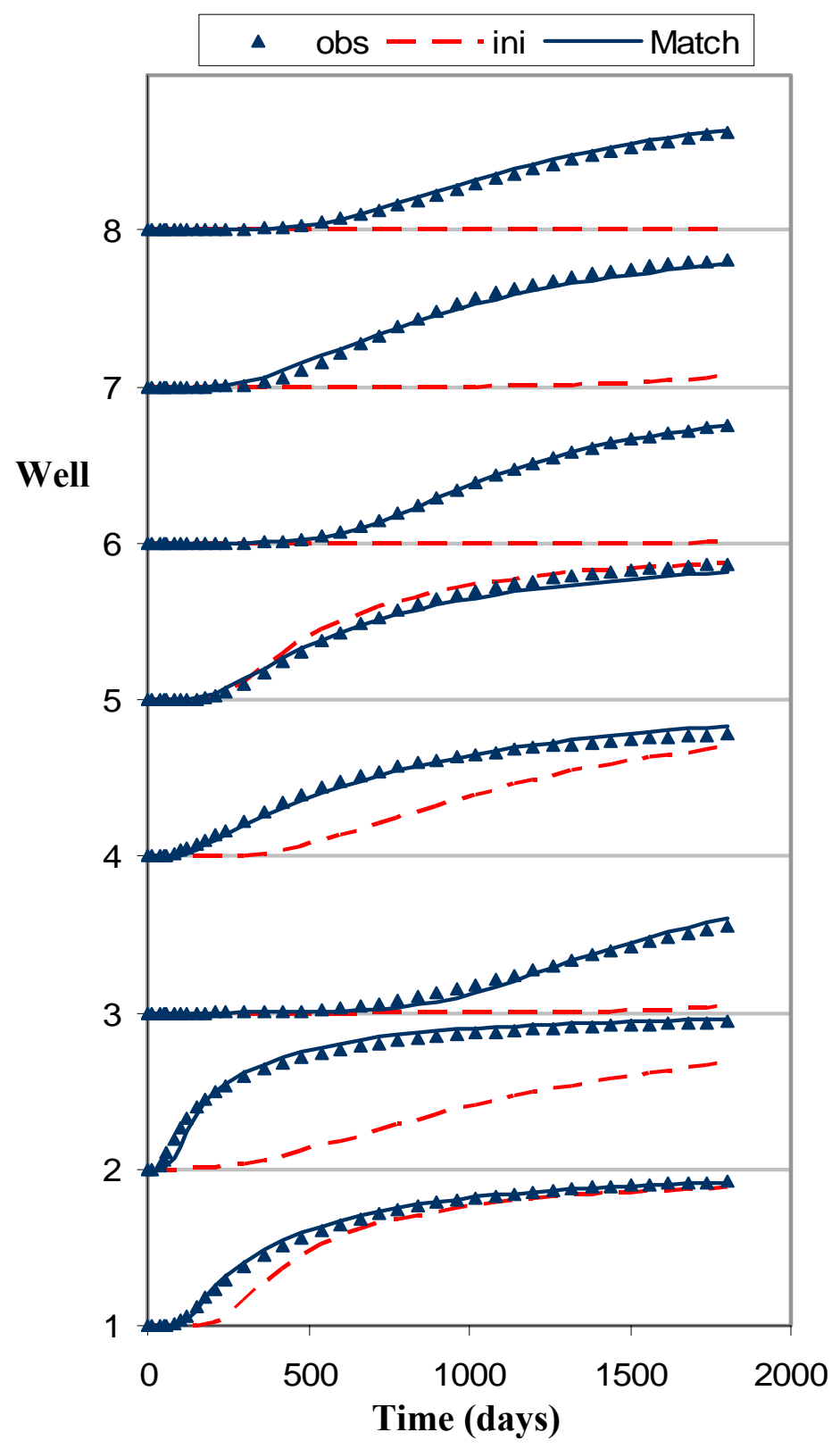

Figure 4.4 - 2D case water cut match 


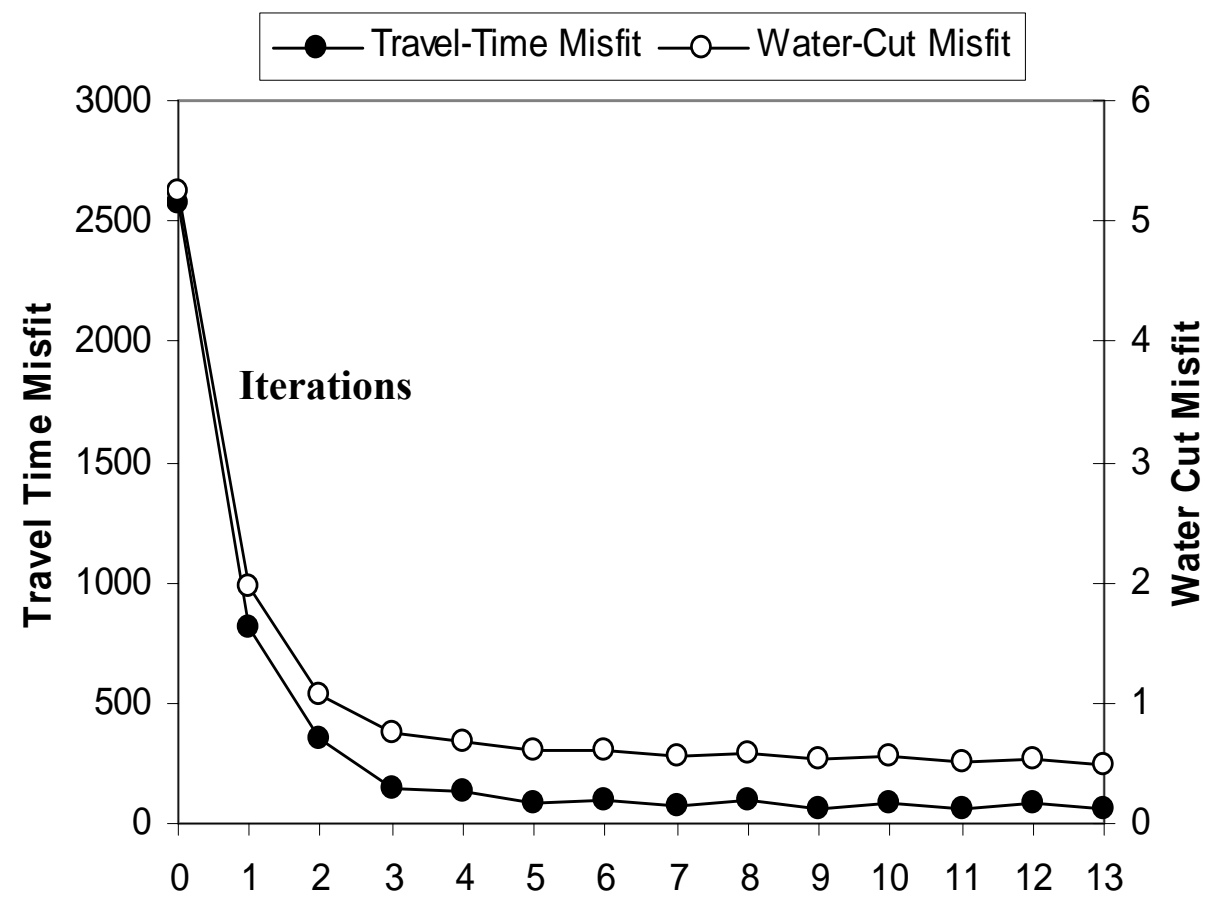

Figure 4.5 - Data misfit vs. iterations 


\subsection{Large Scale 3D Model}

This 3D synthetic example is designed after a carbonate reservoir in west Texas. The dual porosity reservoir model used here has a mesh size of $58 \times 53 \times 10$ with a total of 30,740 grid cells that represent the fracture permeability distribution. To start with, we generated a reference fracture pattern distribution using a discrete fracture network (DFN) model. The DFN model was generated on a layer by layer basis using prespecified distributions that control fracture length, height, aperture and azimuth inside elliptical fracture swarms. The motivation behind using the DFN model is that we can use fracture parameters derived from seismic lineament maps, image logs, regional stress studies etc. to generate realistic fracture distribution constrained to field data. The discrete fracture pattern was then converted to a continuum model using grid block permeability multipliers as discussed before. Figure 4.6 shows the reference fracture permeability for the ten layers. Clearly, the layers 2, 4, 7 and 9 are highly fractured and will have a significant impact on the flow behavior. For comparison purposes, Figure 4.7 shows the discrete fracture networks for layers 2, 4 and 7. The fracture permeability varies over three orders of magnitude from a minimum of $2.5 \mathrm{md}$ to a maximum of 1600 md. The matrix permeability was fixed at $1 \mathrm{md}$.

Figure 4.8 shows relative permeability data for the matrix and fracture systems. The matrix and fracture relative permeability were borrowed from literature ${ }^{12}$ and describes a matrix system with irreducible water saturation of 0.2 and irreducible oil saturation of 0.30 . 


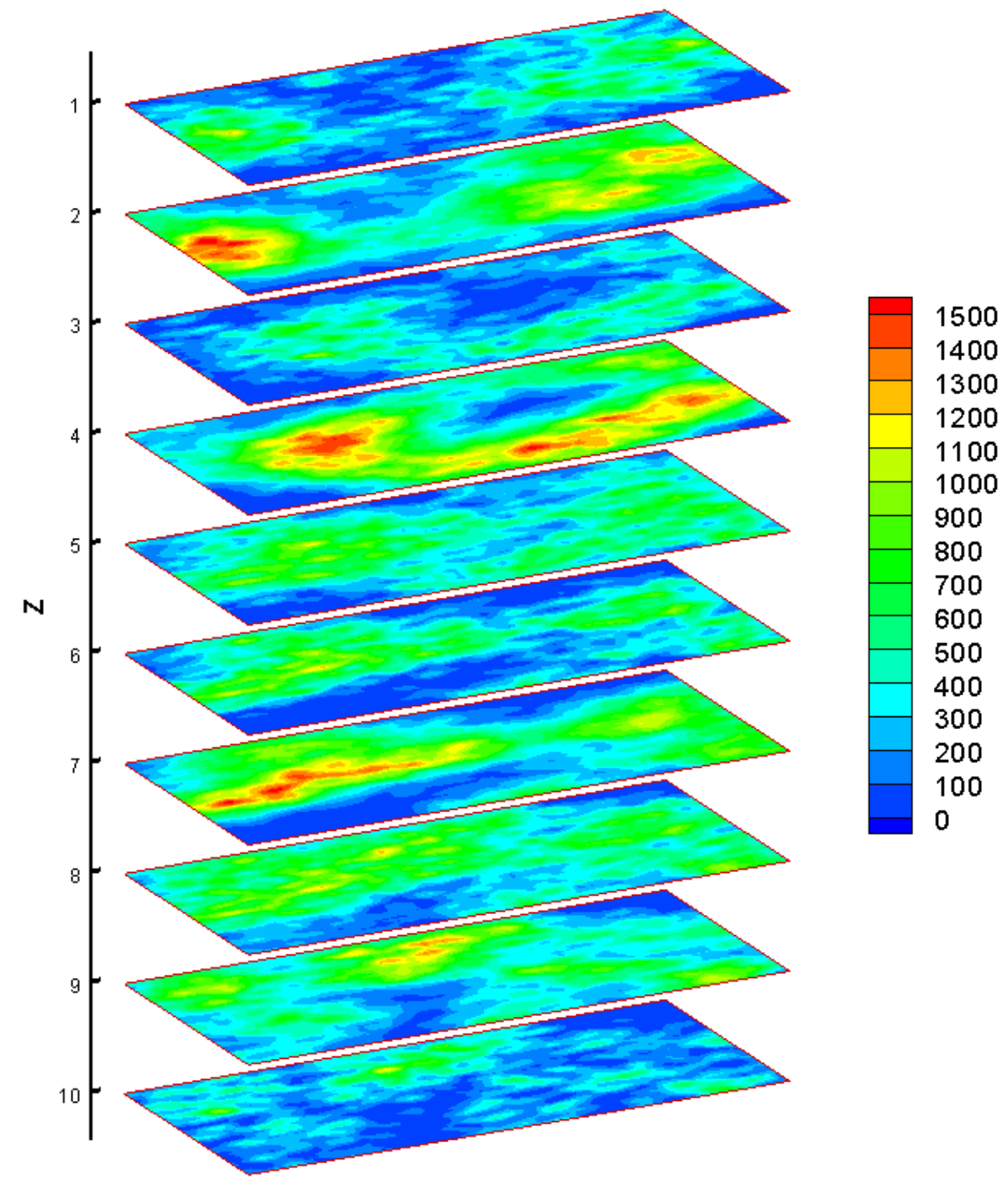

Figure 4.6 - Reference fracture permeability distribution 


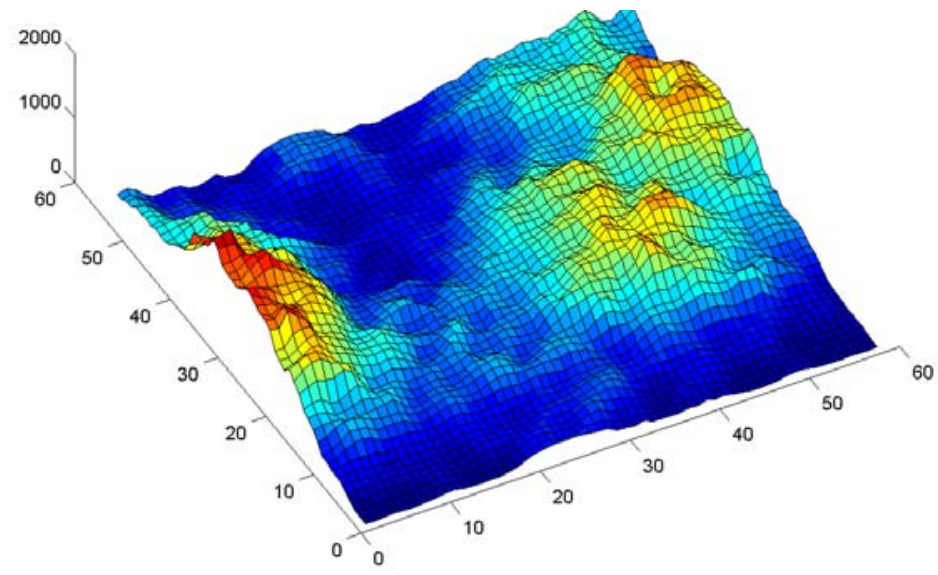

(a) Layer 2

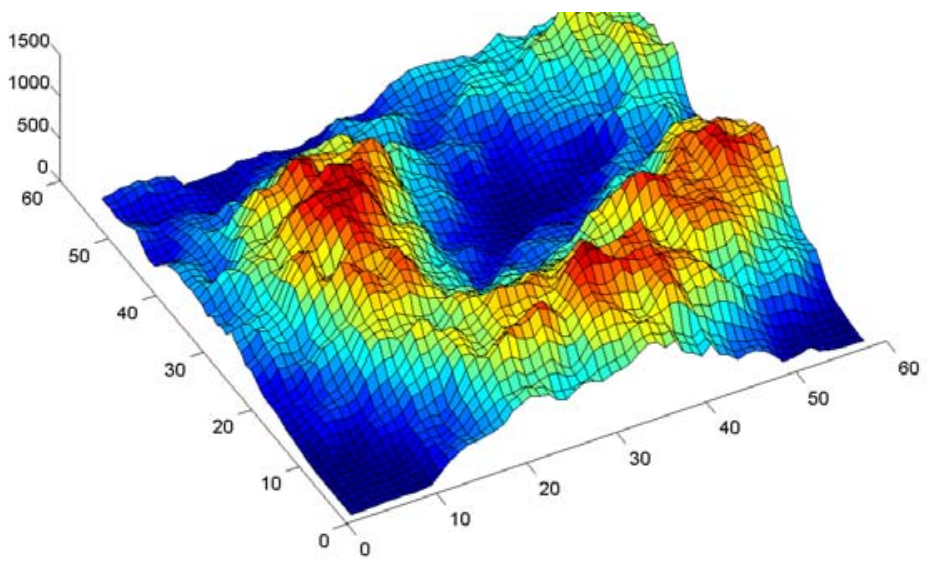

(b) Layer 4
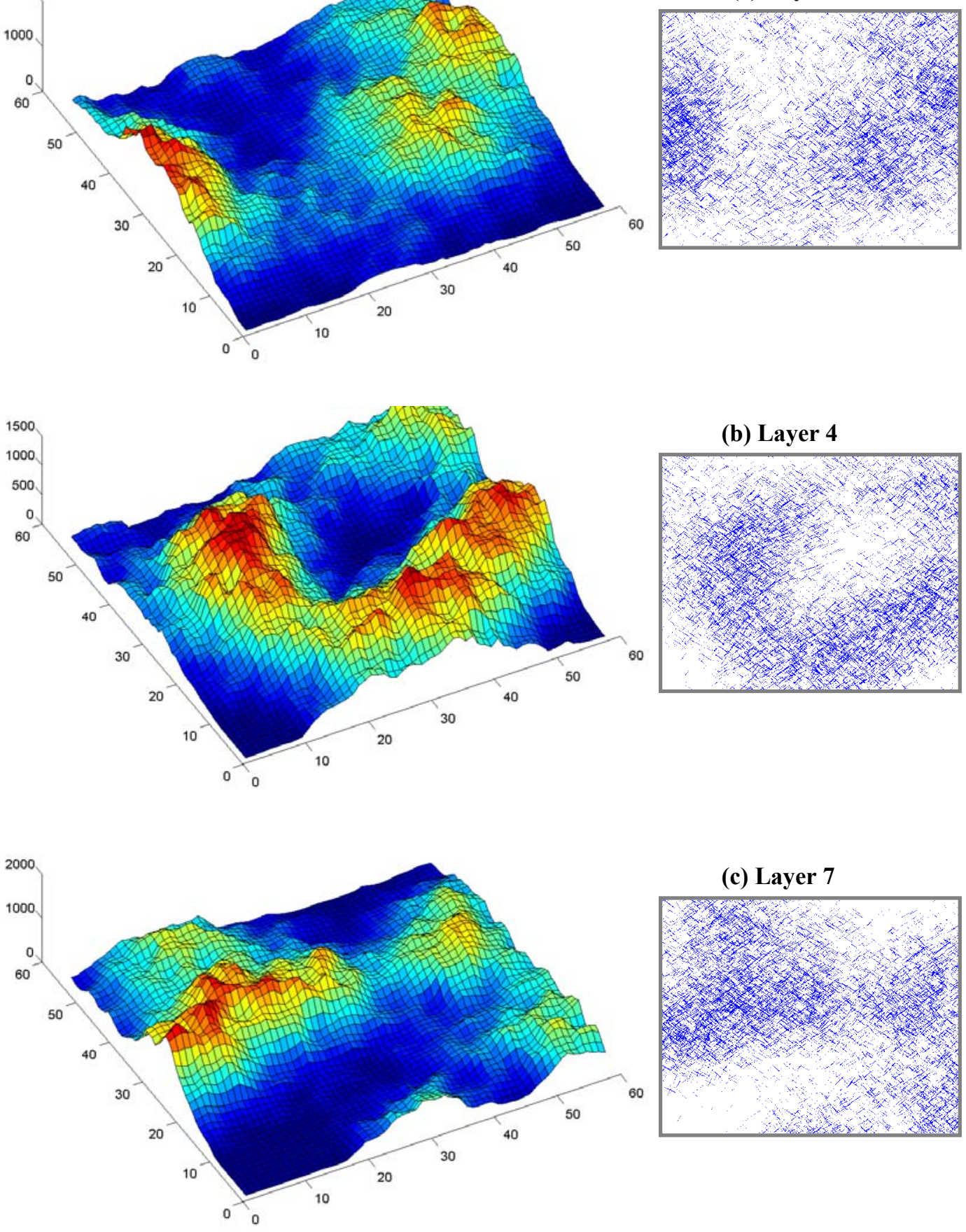

(c) Layer 7

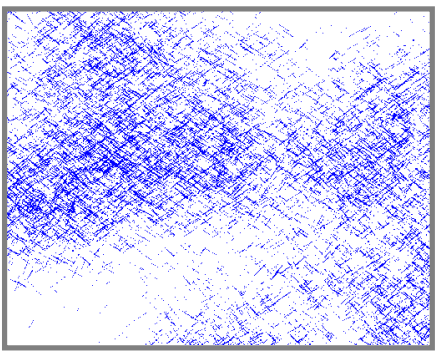

Figure 4.7 - Discrete fracture layers converted to permeability (left panel) using fracture intensity 


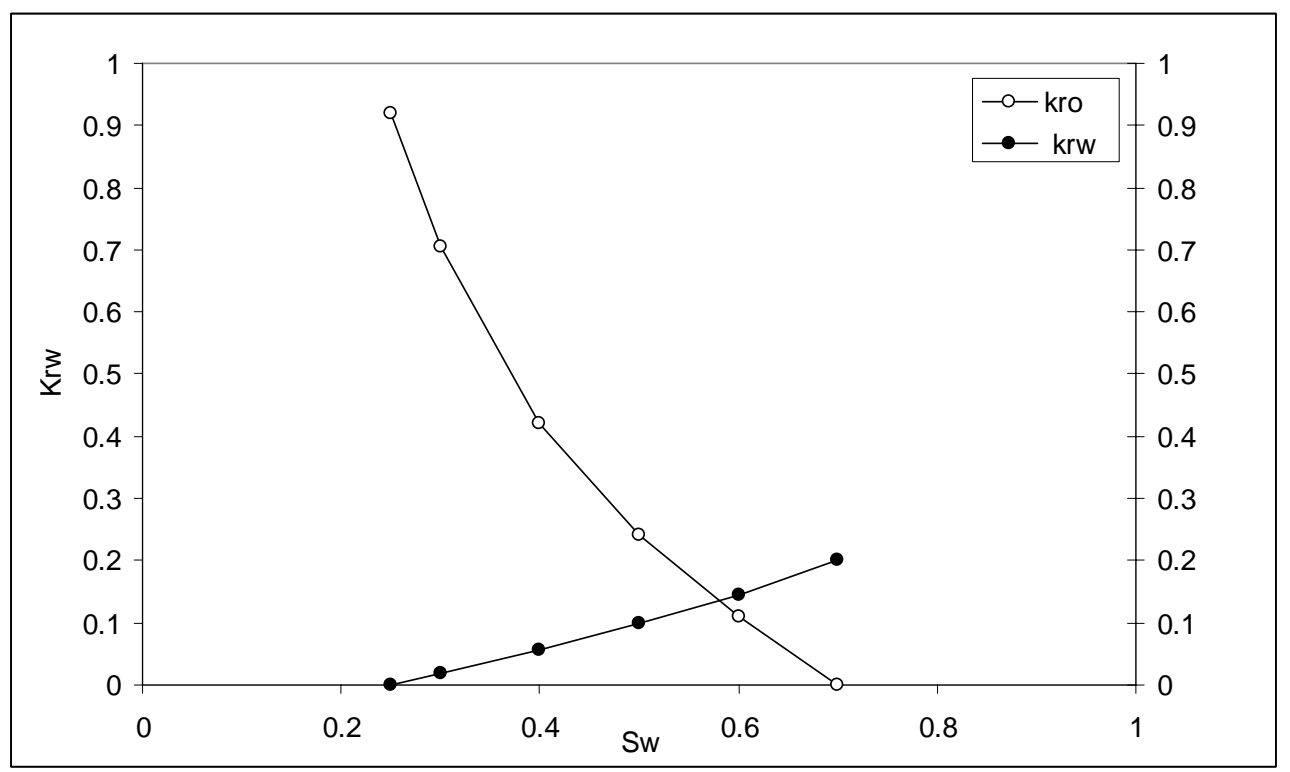

(a) Matrix relative permeability

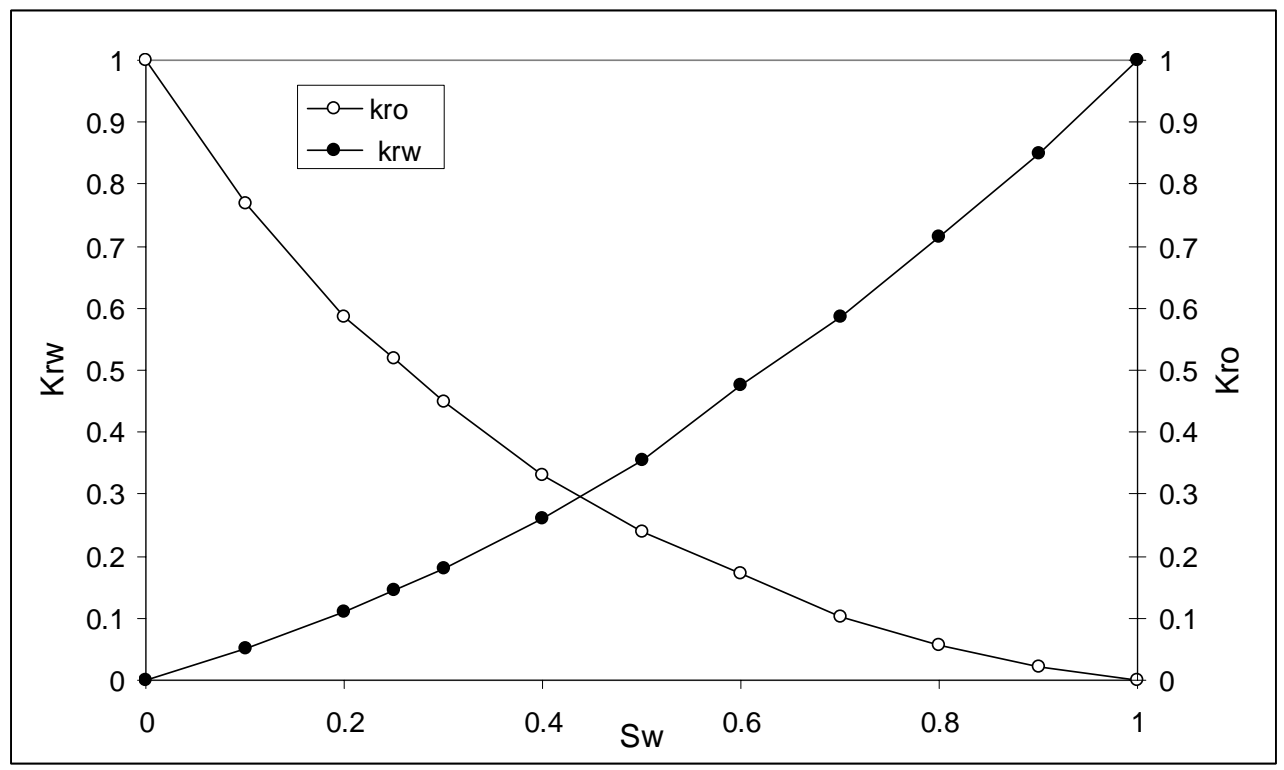

(b) Fracture relative permeability

Figure 4.8 - Relative permeability for matrix and fracture systems 
There are 31 producers and 11 injectors in the model which consist of 11 inverted 5spot patterns covering 320 acres. The detailed production rates and well schedule including infill drilling, well conversion and well shut-ins can be found elsewhere. ${ }^{7}$ Figure 4.9a shows the well locations and the streamlines at the end of 7500 days of simulation. Just as in streamline simulation, we generate the streamlines only when there are significant changes in the well events or boundary conditions. These streamlines are then used to compute the time of flight and travel time sensitivities in Eq. 3.56. For this example we used 11 streamline updates to account for changing well conditions during the sensitivity computations. We have used a commercial finite difference simulator $\left(\right.$ ECLIPSE $^{38}$ ) as a forward model.

For demonstration of our production data integration approach, we will start with two different prior models and match the water-cut history obtained from the reference permeability field. The first model was generated using $50 \%$ of the fractures and fracture swarms in the reference fracture distribution (Figure 4.7). Thus, the prior model contained altogether about a quarter of all the fractures in the reference model. The second model contained $75 \%$ of the fracture and fracture swarm information and thus had approximately half of all the fractures in the reference model. The fracture porosity was kept fixed at 0.03 . 


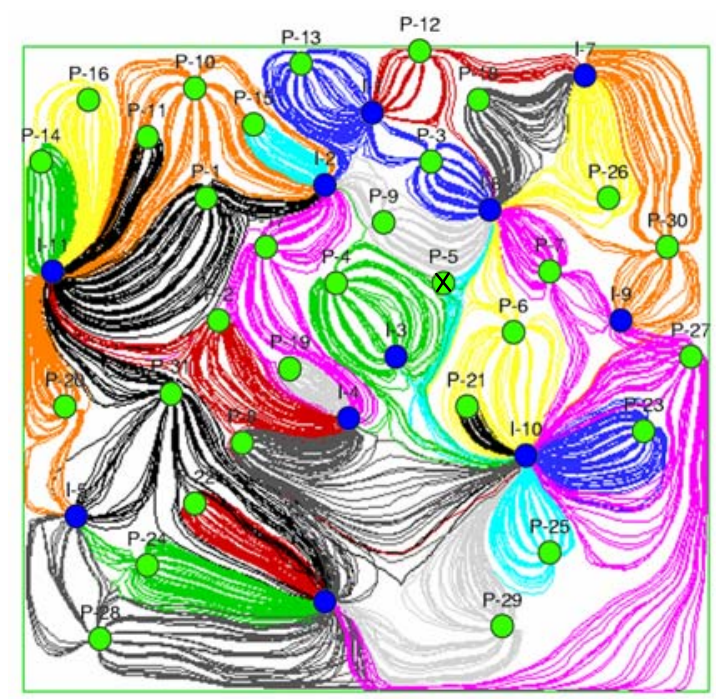

(a) Well locations and streamlines

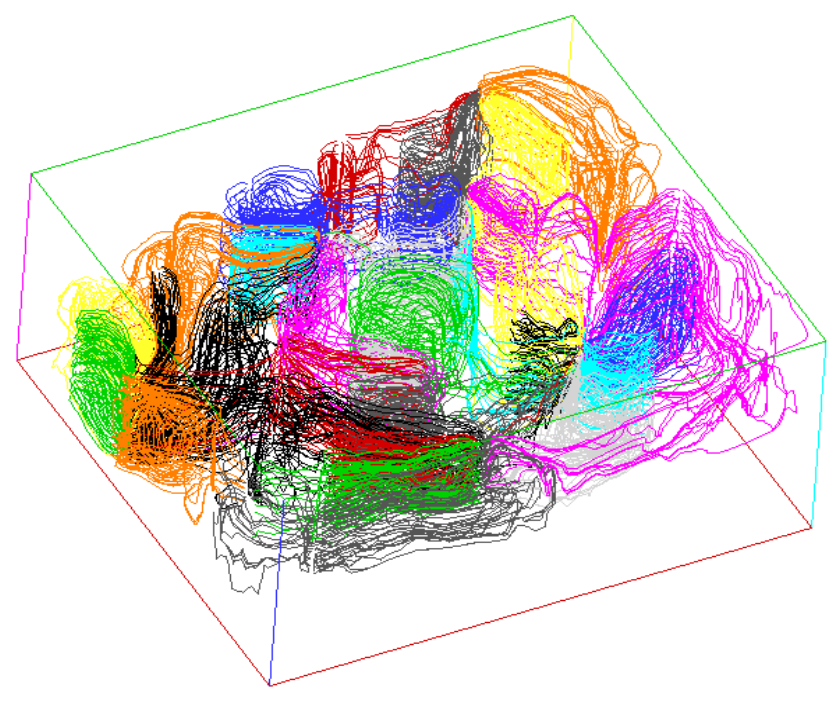

(b) 3D streamlines

Figure 4.9 - (a) Top view shows well locations and streamlines at the end of the last update. (b) 3D streamlines traverse layers in 3D space 


\subsubsection{Prior Model-1: 50\% Fracture Information}

In this example we retain $50 \%$ of the information in the reference fracture pattern (Figure 4.7). Both the fracture swarm location and the fracture density within the swarms were included as part of the prior information. The discrete fracture pattern generated is shown in Figure 4.10 for layers 2, 4 and 7. The prior permeability distribution is shown in Figure 4.11a. As expected, the prior model exhibits less connectivity and fewer preferential flow paths compared to the reference model.

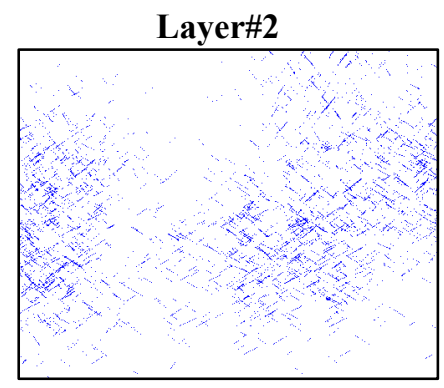

Layer\#4

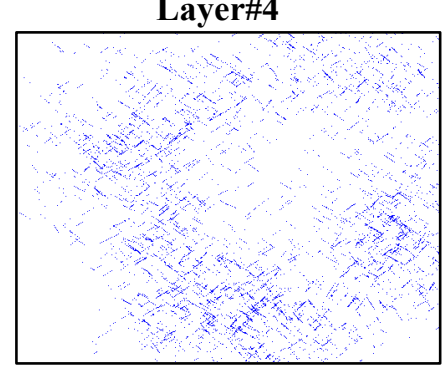

Layer\#7

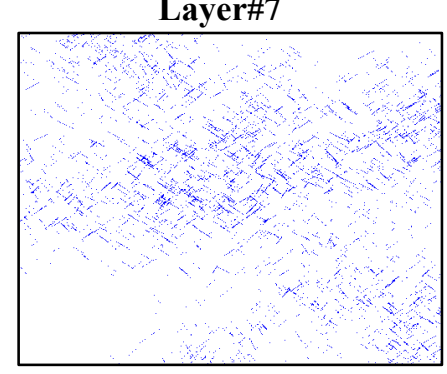

Figure 4.10 - Discrete fracture networks for 3 different layers with $\mathbf{5 0 \%}$ fracture information 


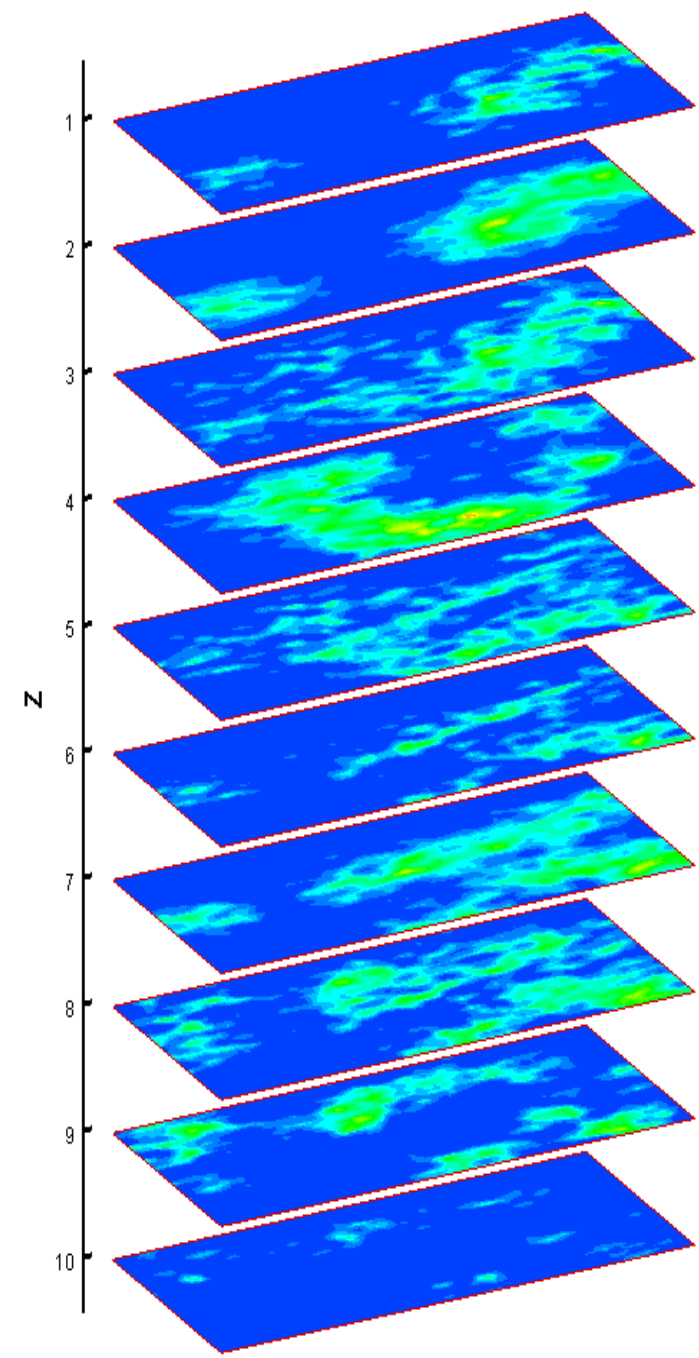

(a) Prior model

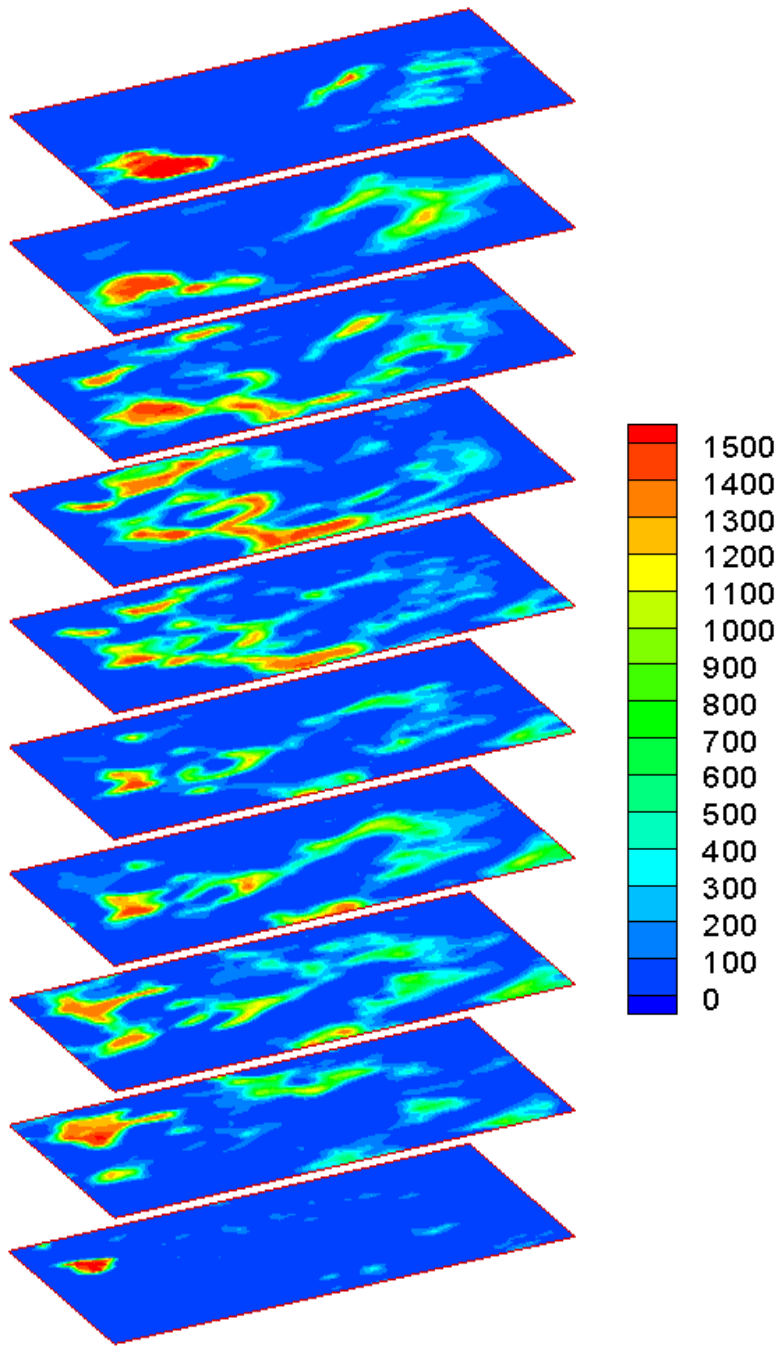

(b) Match model

Figure 4.11 - Permeability distribution with 50\% fracture information 

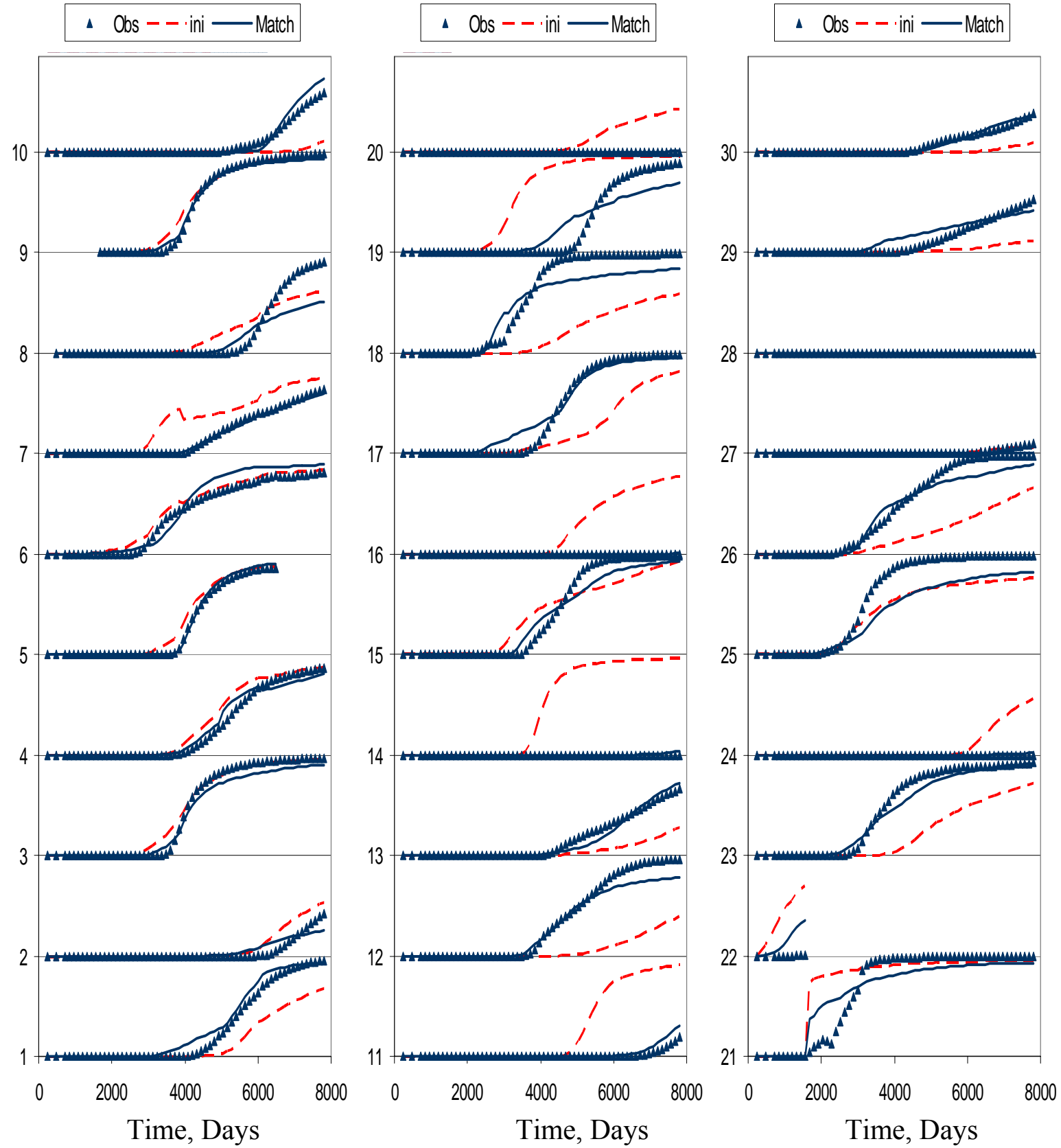

Figure 4.12 - Water cut match and initial response for 30 wells. Almost all the wells showed better water cut match after inversion 
The final permeability field after matching water-cut response at the producers is shown in Figure 4.11b. The water-cut response from the prior model for 30 producers is shown in Figure 4.12. In the same figure we have superimposed the water-cut response from the reference model. Clearly, we see a large discrepancy in the production response because of the lack of fracture connectivity and permeability contrast in the prior model. After inversion, a close agreement is obtained between the reference and the calculated production response as shown in Figure 4.12. On comparison of the final permeability field with the reference permeability distribution, we see that we are able identify the dominant flow paths in the reference model through the integration of production data. For example, in layers 2 and 7 (Figure 4.13), the inversion process re-establishes the high contrast and recovers some of the connected pathways seen in the reference model. We can see similar effects across many of the layers. However, the results also underscore the inherent non-uniqueness in the solution, particularly in 3-D because of the large degree of freedom for these flow paths. This makes prior information vital to the success of the inversion. Finally, Figure 4.14 shows the convergence of the inversion as a function of number of iterations. Both travel time misfit and overall water-cut misfit are reduced significantly after 20 iterations. The entire history matching took 3.2 hours in a PC (Intel Xeon $3.06 \mathrm{GHz}$ Processor). 


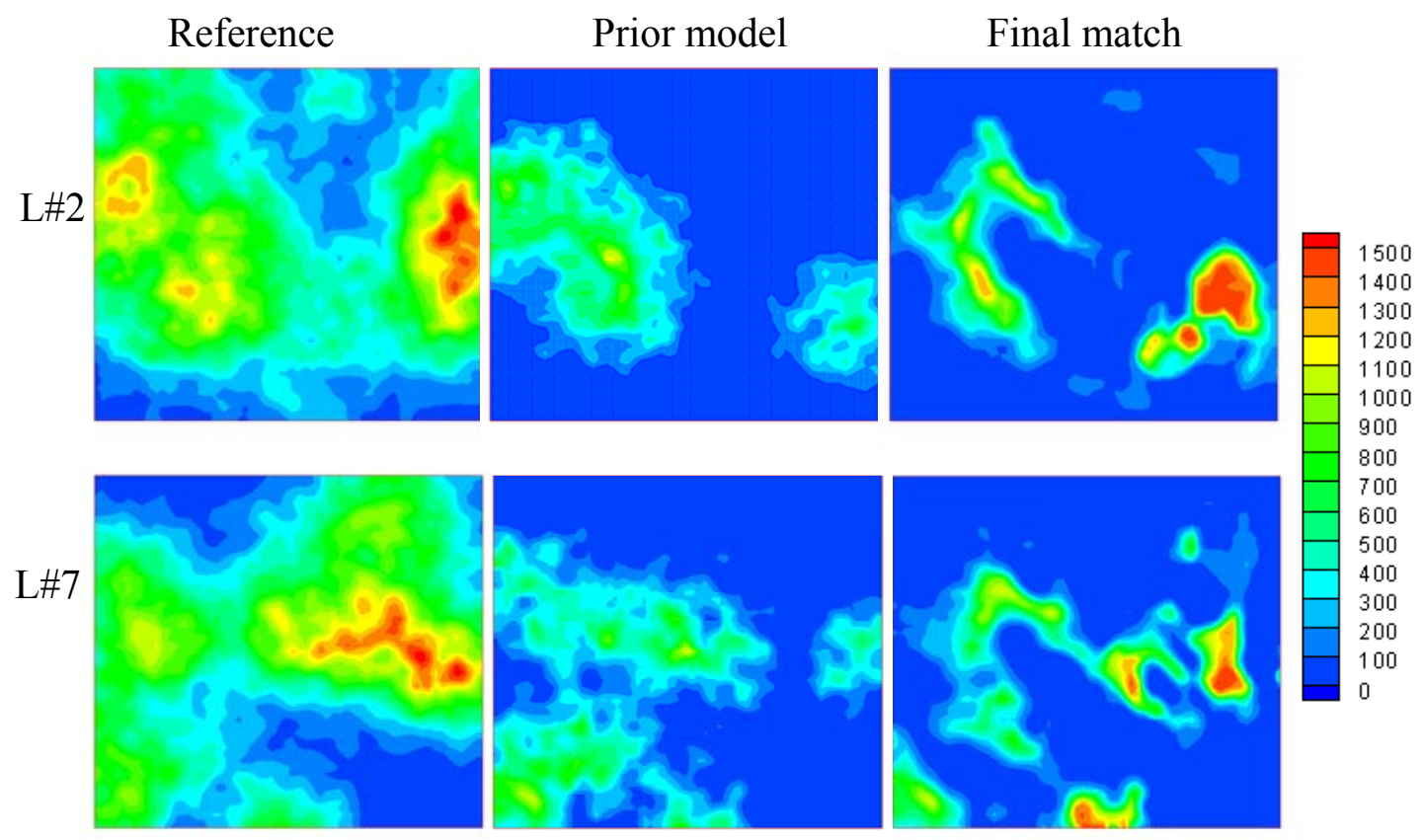

Figure 4.13 - Two layers illustrating that integration of water cut data re-established permeability contrast and identified major flow paths while preserving the prior information 


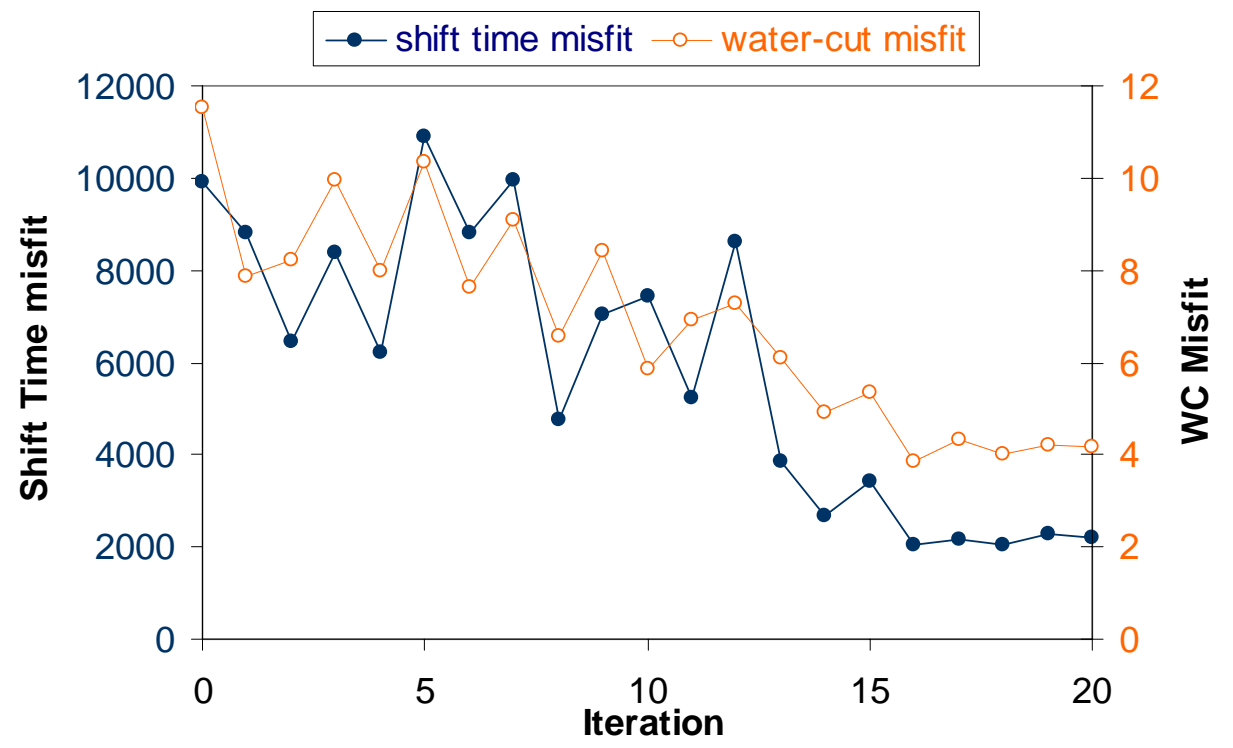

Figure 4.14 - Data misfit vs. iterations (prior model-1) 


\subsubsection{Prior Model-2 : 75\% Fracture Information}

The prior model for this example was generated by retaining $75 \%$ of the information regarding fracture swarms and fracture density within swarms. Again, the discrete fracture network generated for layers 2, 4, and 7 are shown in Figure 4.15. The permeability distribution is shown in Figure 4.16a. As expected, the prior model for this case shows a closer resemblance to the reference permeability field. This is also reflected in the computed water-cut response shown in Figure 4.17. Clearly, the production

\section{Layer\#2}

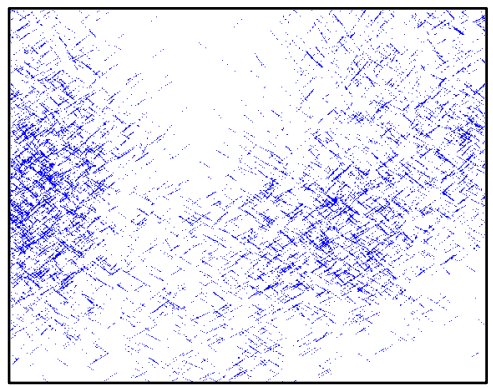

Layer\#4

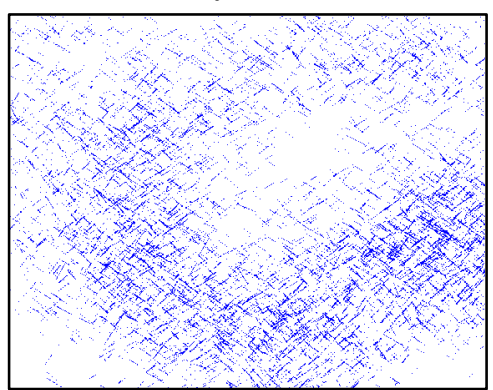

Layer\#7

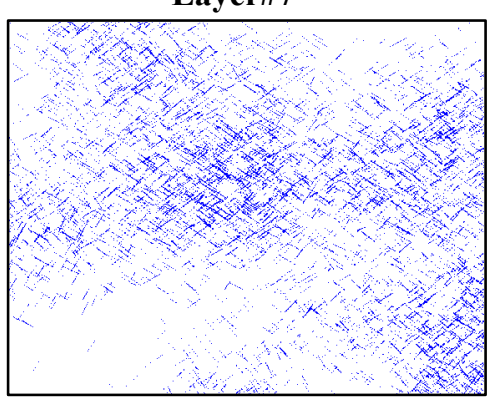

Figure 4.15 - Discrete fracture network for 3 layers with $70 \%$ fracture information 


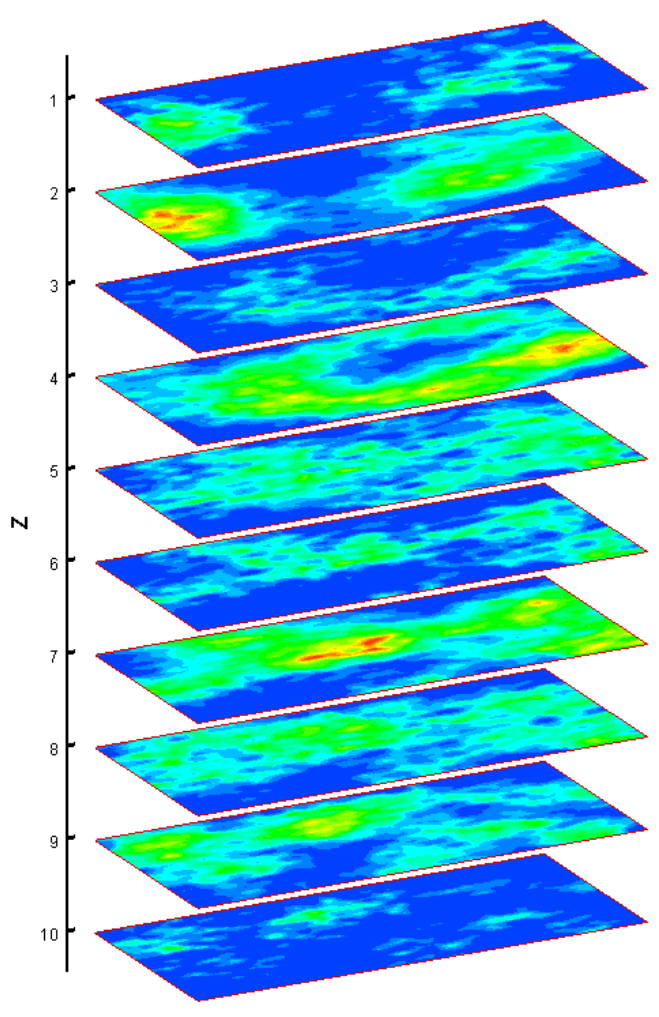

(a) Prior model

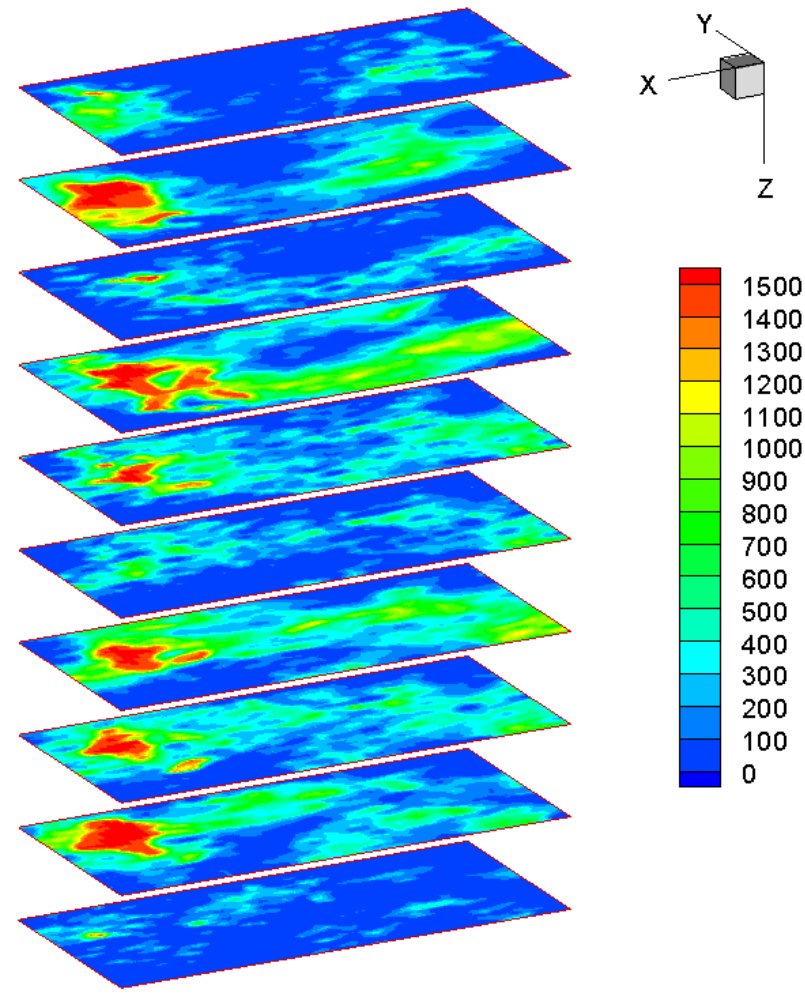

(b) Match model

Figure 4.16 - Permeability distribution for the prior model with $75 \%$ fracture information 
response for this model is much closer to the reference production history compared to the previous model. Although many of the wells show good match, the lack of fracture connectivity and permeability contrast still impacts the production response of several wells, for example, wells 2, 3, 8, 9 and 14, among others. After inversion, we obtain excellent agreement for all wells as shown in Figure 4.17. The final permeability field after inversion is shown in Figure 4.16b. On closer observation, for example, layers 3 and 5 (Figure 4.18), we see that we are able to match the production data with minimum deviation from the prior model. This is expected because of the higher fracture information in the prior model. Also the inverse algorithm by design attempts to preserve prior information to maintain geologic realism. ${ }^{34,39}$ Figure 4.19 shows the misfit reduction as a function of the number of iterations for this example. Again, the misfit is reduced by almost an order of magnitude.

Finally, comparing the results of inversion using the two different prior models, we can clearly see the role of prior information in our ability to predict fluid flow through fractured reservoirs. Although we were able to match the production history reasonably well starting with $50 \%$ fracture information, the results improved significantly when additional fracture data were incorporated. This observation is true for inverse modeling in general; however, the impact is expected to be more pronounced for fractured reservoirs because of the high contrast between the fracture and matrix permeability and the role of preferential fracture flow paths on the overall flow behavior. The inverse problem is ill-posed and we can not expect to reproduce the details of the fracture pattern in the reference model. However, we can reduce the non-uniqueness by anchoring the solution close to the prior model. By starting with different prior models and matching different 'realizations' of the production data, we can explore the uncertainty space by sampling from the posterior distribution. ${ }^{40}$ 

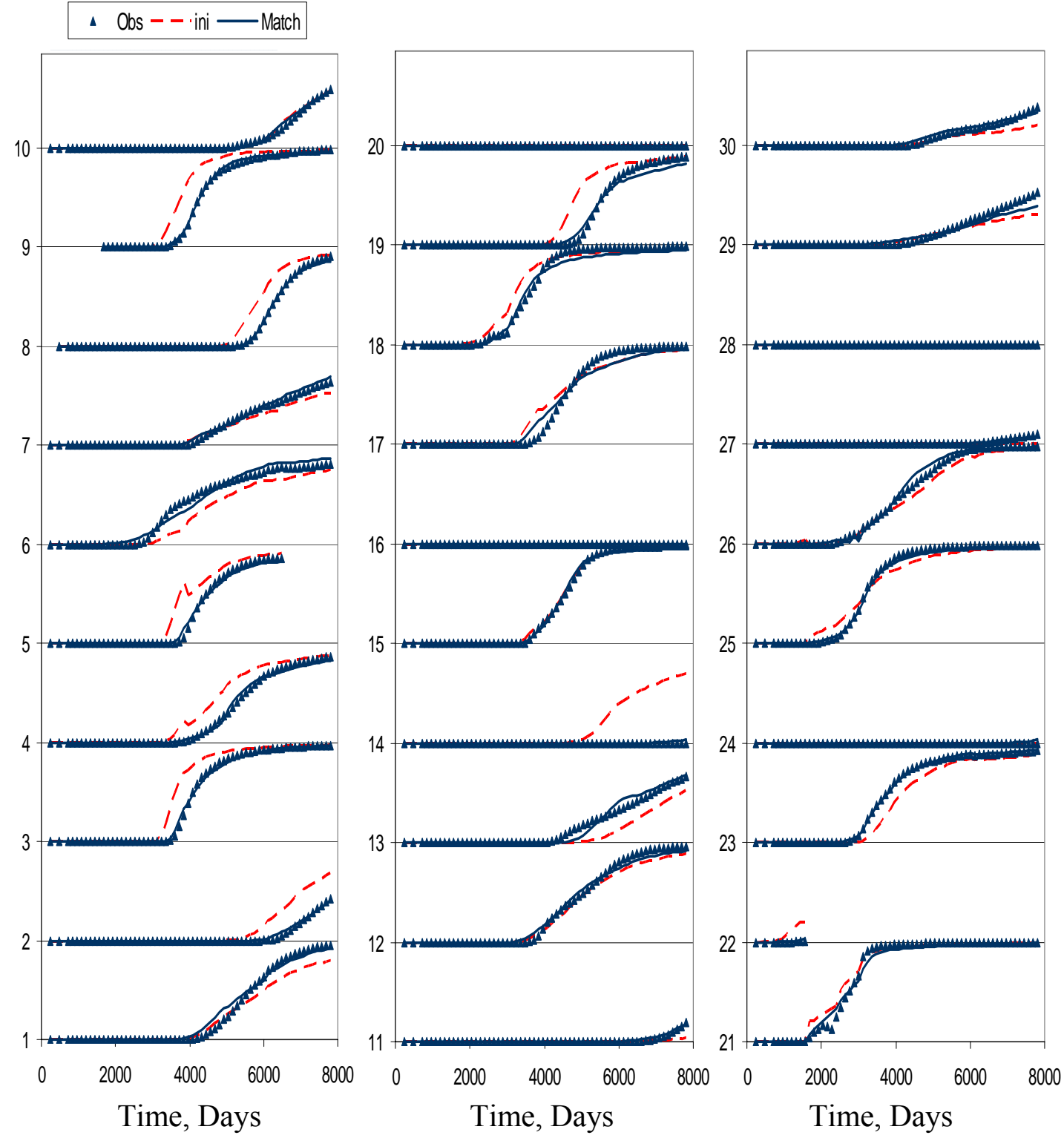

Figure 4.17 - Water cut match and initial response for 30 wells for prior model-2 


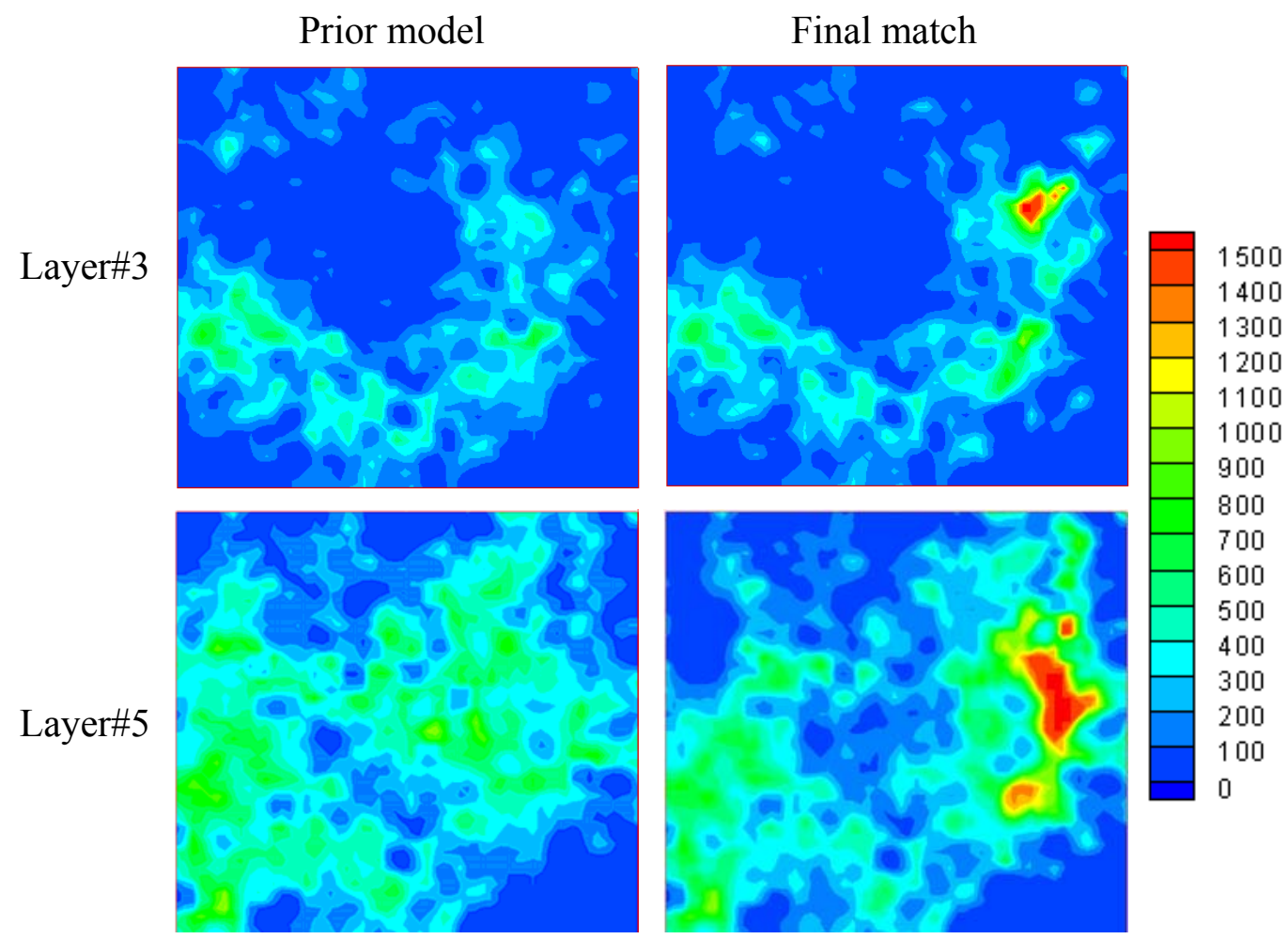

Figure 4.18 - Two layers illustrating changes to the prior model for matching production data. Note that much of the prior model remains unchanged to preserve geologic realism 


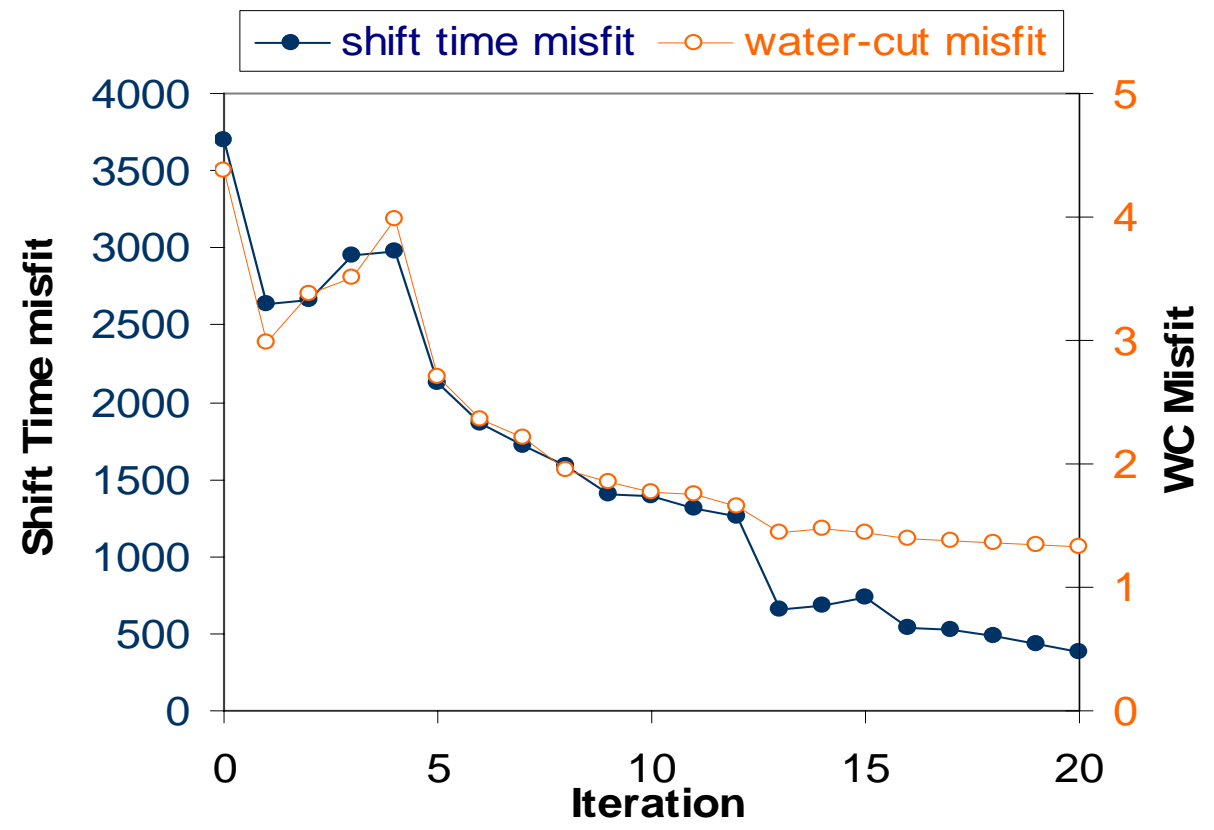

Figure 4.19 - Misfit vs. number of iterations (prior model-2) 


\section{CHAPTER V \\ UNCERTAINTY ASSESSMENT USING APPROXIMATE MCMC}

\subsection{Uncertainty Assessment Methods}

In practice, the uncertainty in reservoir response due to uncertainty in reservoir parameters is evaluated by simulating the response of multiple random realizations that hopefully bracket the underlying variability. Obviously, the validity of such methods is highly dependent of the distribution of the random realizations and if, indeed, they sample the correct distribution of reservoir parameters. Rigorous sampling methods of reservoir parameters, such as MCMC and hybrid MCMC, have been applied successfully by Omre et al. ${ }^{41}$, Oliver et $a l .{ }^{42}$ and Bonet-Cunha et al. ${ }^{43}$. Approximate methods, like Randomized Maximum Liklihood (RML) $)^{44,45}$ and Pilot Point ${ }^{46,47,48}$, attempt to be more efficient than the rigorous methods while providing realizations that approximate the target distribution. All methods require running the flow simulation which, for high resolution models, can be very expensive.

Due to the high computational cost associated with uncertainty assessment methods, several authors have investigated the use of an upscaled model to approximate the high resolution model response. $^{49,50}$ The response from such low resolution models require correction due to errors introduced by upscaling. Variance correction ${ }^{49}$ has been investigated to account for variance reduction in upscaled models and some studies have been done to evaluate the effect of model calibrations from a statistical perspective. ${ }^{51,52}$ Omre and Loedon ${ }^{50}$ have formulated a more rigorous approach to correct for upscaling bias which affects produced volumes and recovery calculations. While such methods try to correct for errors introduced by using a proxy model, in this case an upscaled version, it is clear that upscaling adds more uncertainty to the problem since it requires proper calibration which depends on additional and yet to be determined statistical parameters. 
In this study we developed a highly efficient streamline-based approximate MCMC method that works on high resolution models. The method has a higher acceptance rate than traditional MCMC while eliminating most of the cost associated with rejections. The method works on both streamline and finite difference simulators which makes it applicable to wide range of practical field conditions. This chapter will cover the mathematical formulation of the approximate MCMC method in detail.

\subsection{Mathematical Formulation}

We will cover the theoretical basis of traditional MCMC method and show how it could be extended using an approximate formulation. We will then cover the Gradual Deformation Method (GDM) which is used for generating proposals using an optimized set of basis realizations that insures proper coverage of the reservoir parameter space. A new efficient workflow will be discussed later. Our approach is quite general and is able to handle both streamline and finite difference simulators.

\subsubsection{Traditional MCMC}

In 1953, Metropolis et al. ${ }^{53}$ proposed an algorithm to asymptotically sample a space according to Gibbs-Boltzmann distribution. The algorithm, known as Metropolis Algorithm, followed simple probabilistic rules to perform a biased random walk transition steps. The transition sequence is a Markov chain simulation where the probability of the new state depends only on the previous state. The transition probability insures that after a finite number of transitions, called a burn-in time, the sequence will converge to the desired distribution regardless of the initial starting state. In practical applications such burn-in time can be significant and may require thousands of iterations which make MCMC costly to implement.

In the traditional MCMC algorithm, the following steps are performed:

- Propose an initial state, $m^{1}$.

- Propose a transition state, $m^{*}$, from the parameter pdf, $q\left(\cdot \mid m^{i}\right)$

- Generate $u$ from a uniform distribution $U(0,1)$ 
- Check transition acceptance; if $u \leq \alpha\left(m^{*}, m^{i}\right) \Rightarrow$ promote to proposed state $m^{*}$ otherwise reject $m^{*}$ and propose a new transition state.

There are many acceptance criteria available for our use but the most common and widely used is the Metropolis-Hasting criterion

$$
\alpha=\min \left[1, \frac{q\left(m^{i} \mid m^{*}\right) \cdot f\left(m^{*}\right)}{q\left(m^{*} \mid m^{i}\right) \cdot f\left(m^{i}\right)}\right]
$$

$q(\cdot \mid \cdot)$ is the proposal distribution of transitioning from one state to the next one in the Markov chain sequence and $f(\cdot)$ is the reservoir parameter likelihood distribution. It should be noted that if the chain is symmetric (i.e. the transition from $m^{i}$ to $m^{j}$ has the same probability as the transition from $m^{j}$ to $\left.m^{i}\right)$ then $q(\cdot \mid \cdot)$ will cancel out in Eq. 5.1 leaving only the likelihood distribution, $f(\cdot)$, to be calculated. Also, the transition probability does not depend on the normalization constant. Such formulation lends itself very well to computer iteration.

Production data is related to reservoir parameters through the relationship, $d=g(m)$, where $g$ is a nonlinear transfer function that calculates reservoir response. Typically, finite difference or streamline simulators are used. If we assume that production data and reservoir parameters follow a multi-Gaussian distributions, the posterior distribution can be modeled using the following relationship ${ }^{17,19}$

$$
\begin{aligned}
f(m) \propto \exp [ & -\frac{1}{2}\left(m-m_{\text {prior }}\right) \mathbf{C}_{M}^{-1}\left(m-m_{\text {prior }}\right) \\
& \left.-\frac{1}{2}\left(g(m)-d_{o b s}\right) \mathbf{C}_{D}^{-1}\left(g(m)-d_{o b s}\right)\right]
\end{aligned}
$$

where $m_{\text {prior }}$ is the prior model, $\mathbf{C}_{M}$ is the parameter covariance, $\mathbf{C}_{D}$ is the data covariance and $d_{o b s}$ is the observed response. Notice that the proportionality constant will vanish in Eq 5.1. 


\subsubsection{Approximate MCMC}

Fox and Nicholls ${ }^{20}$ proposed a method to perform MCMC using approximate likelihood calculation for the acceptance criteria in a modified Metropolis-Hastings algorithm. In their proposed procedure, if a proposal is accepted, the exact likelihood is calculated as in traditional MCMC but if the sample is rejected then a new sample is drawn and the procedure is iterated. The steps are exactly the same as traditional MCMC with the exception of adding the approximate likelihood check for rejection which filters out rejected samples before performing exact acceptance check. The procedure does not compromise the rigorousness of traditional MCMC but simply eliminates most of rejected samples and avoid paying the cost of running the exact MCMC except for samples that have a higher chance of being accepted.

As discussed in chapter III, the streamline analytical sensitivity defines the change in travel time response as a function of change in a given reservoir parameter,

$$
\frac{\partial t}{\partial m}=\frac{\frac{\partial \tau}{\partial m}}{\frac{\partial f_{w f}}{\partial S_{w f}}}
$$

Let us denote the change in values in transition from $m^{i}$ to $m^{*}$ by $\delta m=m^{*}-m^{i}$. For small perturbation to reservoir parameters, $\delta m$, we are going to make the following linearized approximation,

$$
g^{*}\left(m^{*}\right)=g^{*}\left(m^{i}+\delta m\right) \simeq g\left(m^{i}\right)+\mathbf{G} \delta m
$$

where $\mathbf{G}$ is the sensitivity given by Eq. 5.3 and $g^{*}\left(m^{*}\right)$ is the approximation to the reservoir response corresponding to $\mathrm{m}^{*}$. Using Eq. 5.4, we can rewrite the acceptance criteria in Eq. 5.1 


$$
\alpha=\min \left[1, \frac{q\left(m^{i} \mid m^{*}\right) \cdot f^{*}\left(m^{*}\right)}{q\left(m^{*} \mid m^{i}\right) \cdot f\left(m^{i}\right)}\right]
$$

where $f^{*}\left(m^{*}\right)$ is given by

$$
\begin{aligned}
f^{*}\left(m^{*}\right) \propto \exp [ & -\frac{1}{2}\left(m-m_{\text {prior }}\right) \mathbf{C}_{M}^{-1}\left(m-m_{\text {prior }}\right) \\
& \left.-\frac{1}{2}\left(g^{*}\left(m^{*}\right)-d_{o b s}\right) \mathbf{C}_{D}^{-1}\left(g^{*}\left(m^{*}\right)-d_{o b s}\right)\right]
\end{aligned}
$$

\subsubsection{Parameter Perturbation Using Gradual Deformation}

There many methods proposed for sampling from parameter space. Some local perturbation methods use two points swapping where two parameter values from two different grid blocks are swapped to generate a proposal. Global perturbation methods perturb all parameters in the model for each proposal generated. Oliver et $a .^{42}$ have showed that local perturbation is more efficient for generating realizations for Markov chains when the problem is highly nonlinear while global perturbation is more efficient for linear or slightly nonlinear problems. Since the approximation we derived for approximate MCMC linearizes the problem locally, global perturbation will be the more efficient approach.

The gradual deformation method ${ }^{54}$ provides an excellent framework for performing global perturbation. The method was originally developed to gradually deform or change Gaussian related stochastic reservoir models while preserving their covariance structure. The method has been later extended to non-Gaussian mixtures ${ }^{55}$ and has been applied to a wide range of problems including dynamic data integration and uncertainty assessment.

Let $Z(x)$ be a multi-Gaussian random function known at $N$ locations $x_{n}$ where $n=1,2, . ., N$ with a covariance $\rho(h)$. Let $S_{i}(x)$ be a series of independent standard multi-Gaussian random functions with the same covariance function as $Z(x)$. We can construct conditional random functions using the following, 


$$
Y_{i}(x)=Z^{*}(x)+S_{i}(x)-S_{i}^{*}(x) \quad \forall i=1,2, \ldots, I
$$

where $Z^{*}(x)$ is simple kriging of $Z(x)$,

$$
Z^{*}(x)=\sum_{n} \lambda_{n}(x) Z\left(x_{n}\right)
$$

and $S_{i}^{*}(x)$ is simple kriging of $S_{i}(x)$,

$$
S_{i}^{*}(x)=\sum_{n} \lambda_{n}(x) S_{i}\left(x_{n}\right)
$$

and the kriging weights are given by the kriging system,

$$
\sum_{i=1}^{N} \lambda_{i}(x) \rho\left(x_{n}-x_{i}\right)=\rho\left(x_{n}-x\right) \quad \forall n=1,2, \ldots, N
$$

Journel and Huijbregts ${ }^{56}$ have shown that $Y_{i}(x) \forall i=1,2, \ldots, I$ are series of standard multi-Gaussian random functions with the same covariance as $Z(x) . Y_{i}(x)$, however, are not independent as they are conditioned to the same random variable, $Z\left(x_{n}\right)(n=1,2, \ldots, N)$. The cross-covariance of a pair $Y_{i}(x)$ and $Y_{j}(x)$ where $i \neq j$ depends on the location $x$ and $x+h$ and given by the following,

$$
\begin{aligned}
E\left[Y_{i}(x) Y_{j}(x+h)\right] & =E\left[Z^{*}(x) Z^{*}(x+h)\right] \\
& =\sum_{i, n} \lambda_{i}(x) \lambda(x+h) \rho\left(x_{i}-x_{n}\right) \\
& =\sum_{n} \lambda_{n}(x) \rho\left(x_{n}-x-h\right) \\
& =\sum_{n} \lambda_{n}(x+h) \rho\left(x_{n}-x\right)
\end{aligned}
$$

A linear combination of $Y_{i}(x)$ will also be a multi-Gaussian random function, 


$$
Y(x)=\sum_{i} \alpha_{i} Y_{i}(x)
$$

We can combine Eq. 5.7 and Eq. 5.12,

$$
Y(x)=Z^{*}(x) \sum_{i} \alpha_{i}+\sum_{i} \alpha_{i}\left[S_{i}(x)-S_{i}^{*}(x)\right]
$$

Examining Eq. 5.13, it is clear that for $Y(x)$ to be conditioned to the data vector, $Z\left(x_{n}\right)(n=1,2, \ldots, N)$, the weights has to sum to unity,

$$
\sum_{i} \alpha_{i}=1
$$

Eq. 5.14 is the conditioning constraint. The covariance of $Y(x)$ can be written with the conditioning constraint as follows,

$$
E[Y(x) Y(x+h)]=\rho(h) \sum_{i} \alpha_{i}^{2}+\rho^{*}(x, x+h)\left(1-\sum_{i} \alpha_{i}^{2}\right)
$$

Examining Eq. 5.15, we can deduce that $Y(x)$ will have the same covariance of $Y_{i}(x)$ under the following constraint,

$$
\sum_{i} \alpha_{i}^{2}=1
$$

Eq. 5.16 is the covariance constraint. When the two constraints above are satisfied, the linear combination given by Eq. 5.12 will preserve the covariance structure. The set of weights to satisfy the constraints can be obtained parametrically by finding the intersection of a hyper plane and a hyper sphere in multidimensional space. For the 
simple case of a combination of three models, the parametric form of the weights under the constraints $\sum_{i}^{3} \alpha_{i}^{2}=1$ and $\sum_{i}^{3} \alpha_{i}=1$ are given by

$$
\begin{aligned}
& \alpha_{1}=\frac{1}{3}+\frac{2}{3} \cos (t) \\
& \alpha_{2}=\frac{1}{3}+\frac{2}{3} \sin \left(-\frac{\pi}{6}+t\right) \quad t \in(-\pi, \pi) \\
& \alpha_{3}=\frac{1}{3}+\frac{2}{3} \sin \left(-\frac{\pi}{6}-t\right)
\end{aligned}
$$

For the case of independent realizations, only the covariance constraint is needed and a general form for combination of $M+1$ realizations is given by

$$
Y(x)-m_{Y}=\sum_{i=0}^{M} \alpha_{i}\left(Y_{i}(x)-m_{Y}\right)
$$

where we deform the residuals around the mean, $m_{Y}$. The weights satisfy the covariance constraint for any choice of $t_{i}$

$$
\begin{aligned}
& \alpha_{0}=\prod_{i=1}^{m} \cos \left(t_{i}\right) \\
& \alpha_{i}=\sin \left(t_{i}\right) \prod_{j=i+1}^{m} \cos \left(t_{j}\right) \quad t_{i} \in(-\pi, \pi) \\
& \alpha_{m}=\sin \left(t_{m}\right)
\end{aligned}
$$

\subsubsection{Selection of Basis Realizations}

While gradual deformation works on any set of realizations with the same covariance structure, care need to be taken when selecting the set of basis realizations to be used for sampling. Liu and Oliver ${ }^{57}$ assessed the efficiency of the GDM in sampling from an attribute space using two realizations mixtures and a simple linear model to minimize 
simulation cost. They concluded that the GDM does produced an acceptable distribution when compared with other sampling methods and compared very well with the MCMC distribution which is known to reflect the true distribution. They raised concerns about the method efficiency in generating proposals due to the need to add random realizations during the inner iterations which introduce an arbitrary search direction that may not be favorable. They also concluded that using a larger number of realizations will improve efficiency dramatically. In our method, we are using GDM to generate proposals for high resolution models and efficiency is a major concern. To address these concerns and design an efficient sampling algorithm, several decisions were made. First, we are going to use a mixture of a large number of stochastic realizations to improve convergence and sampling efficiency and eliminate the need to introduce random realizations within the iteration loop. Second, since the iteration will be performed without updating search directions, we need to choose an "optimum basis" of realizations that insure proper coverage of the parameter space.

A set of realizations are considered optimum if their GD mixture "span" the parameter space. We know from linear algebra that for an $\mathrm{N}$ dimensional space, we need $\mathrm{N}$ spanning vectors or realizations in our case. What we are attempting to find is an optimum set, i.e. a set of size $M<<N$ that maximize the coverage of the $\mathrm{N}$ dimensional parameter space. The procedure will start by generating a large number of stochastic realizations using geostatistical algorithm like Sequential Gaussian Simulation. Next, we are going to extract only a subset of size $M$ of those stochastic models that provide the maximum coverage of parameter space. These models will be used as basis models in GDM for generating proposals.

Generating such an optimum basis requires special attention. Starting with a large set of stochastic models, $Y_{i}(x)(i=1,2, \ldots, K)$, we want first to extract a subset $M$, $\hat{Y_{i}}(x)(i=1,2, \ldots, M<K)$, that maximize the sum of difference norm among all possible combinations of M. Mathematically,

$$
\operatorname{Obj}=\operatorname{Max}\left(\sum_{i=1}^{M-1} \sum_{j=1}^{M}\left\|m_{i}-m_{j}\right\|_{i \neq j}\right) \quad M \in K
$$


It should be noted that the difference norm matrix is triangular of size $\mathrm{K}^{*} \mathrm{~K}$. Once the difference norm matrix is calculated, the optimization becomes independent of model size and dependent only on the number of models. The resulting optimized subset $M$ will give the boundary points of the stochastic scatter cloud. Let us examine a simple case of 2-dimensional model to gain insight into the process. If we assume that we have a standard bi-Gaussian random variable, we can draw random 100 points (models) from the bi-Gaussian distribution. The result is shown in Figure 5.1.

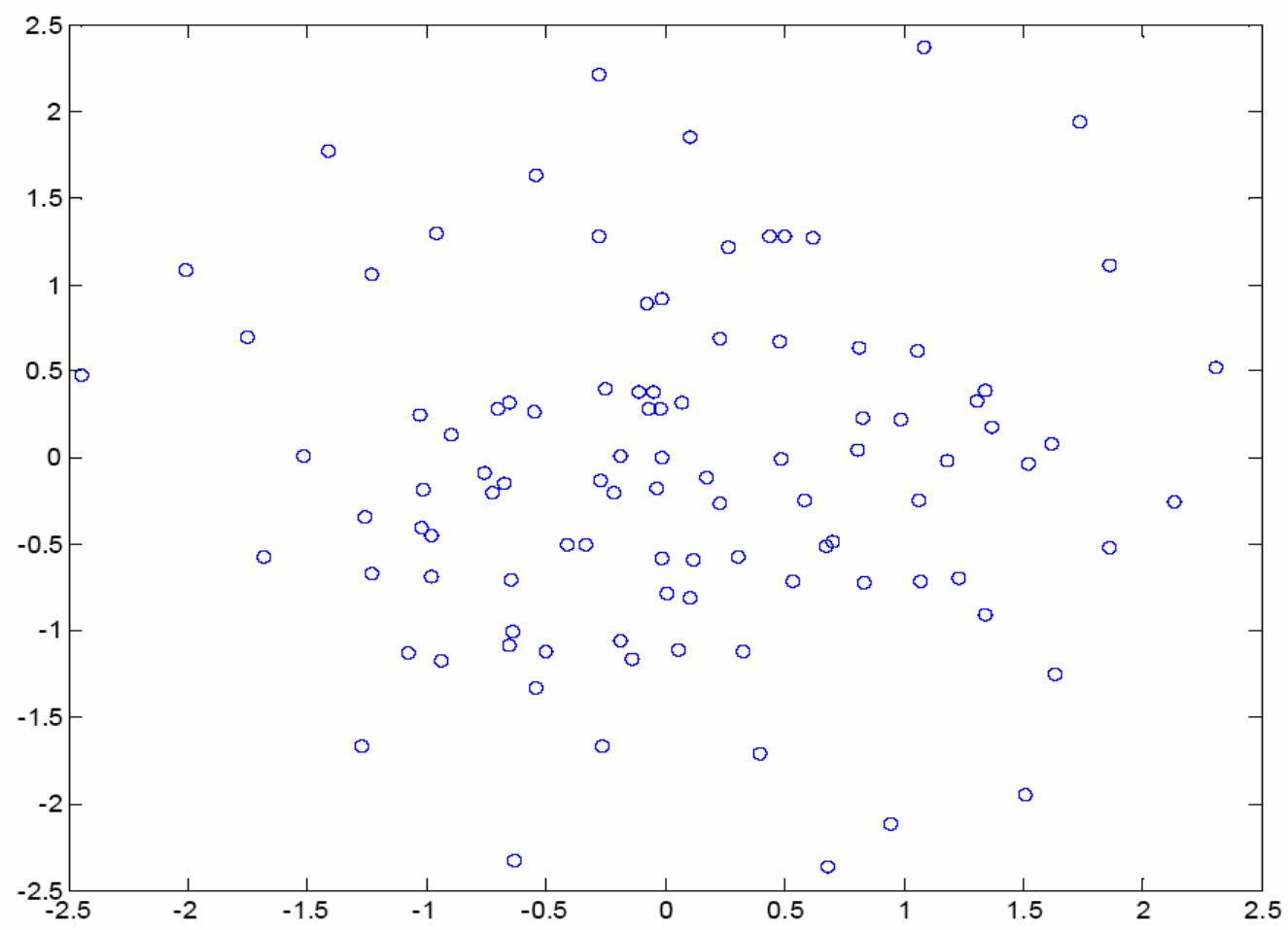

Figure 5.1 - Drawing 100 bi-Gaussian points 


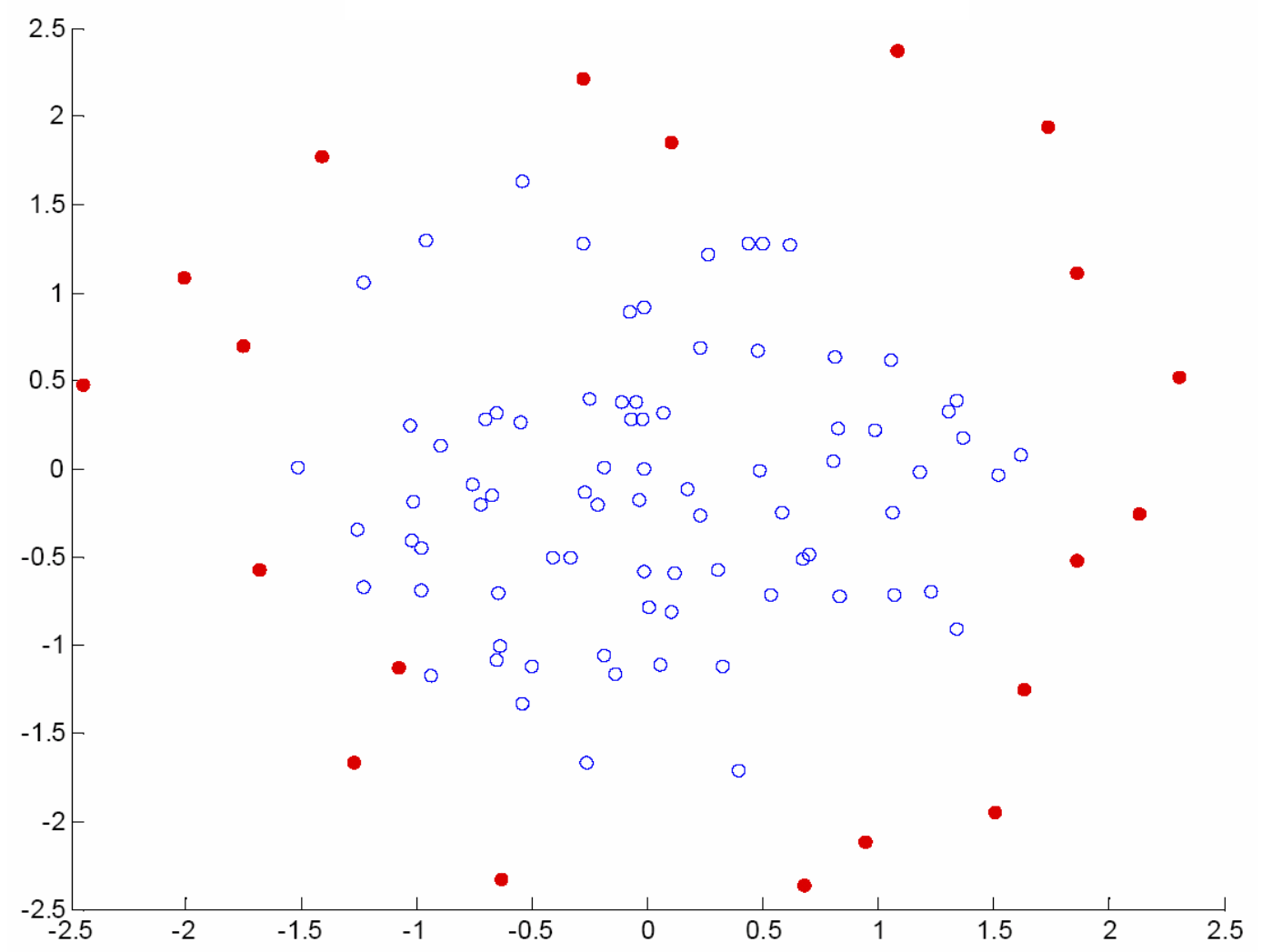

Figure 5.2 - 20 points subset from Eq. 5.20 


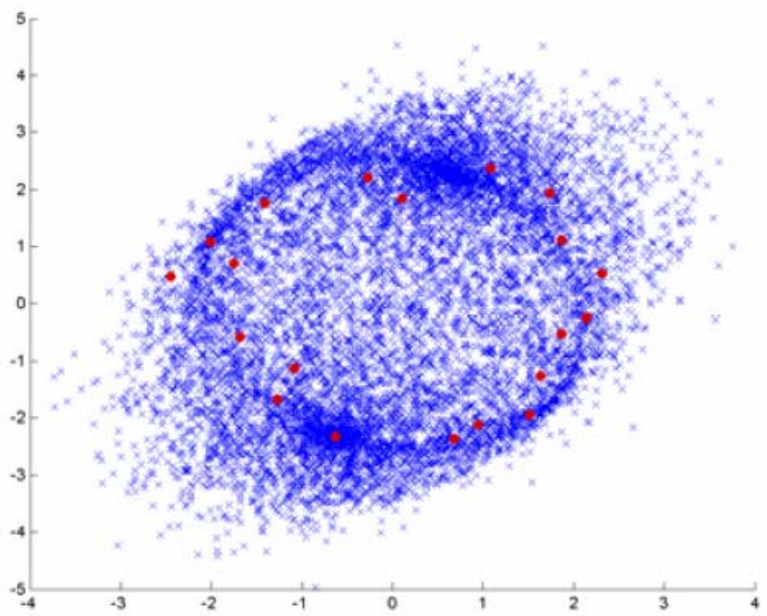

(a) 20 points basis subset

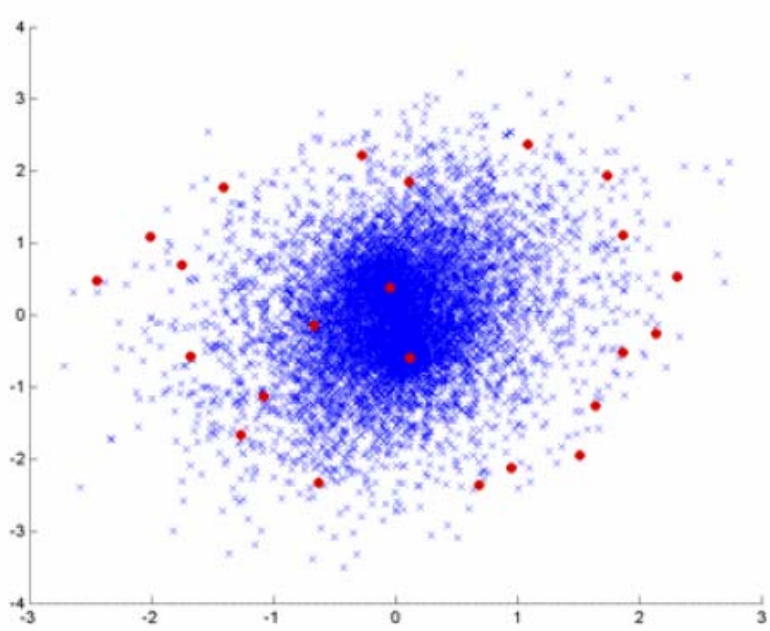

(b) 20 points +3 interior points

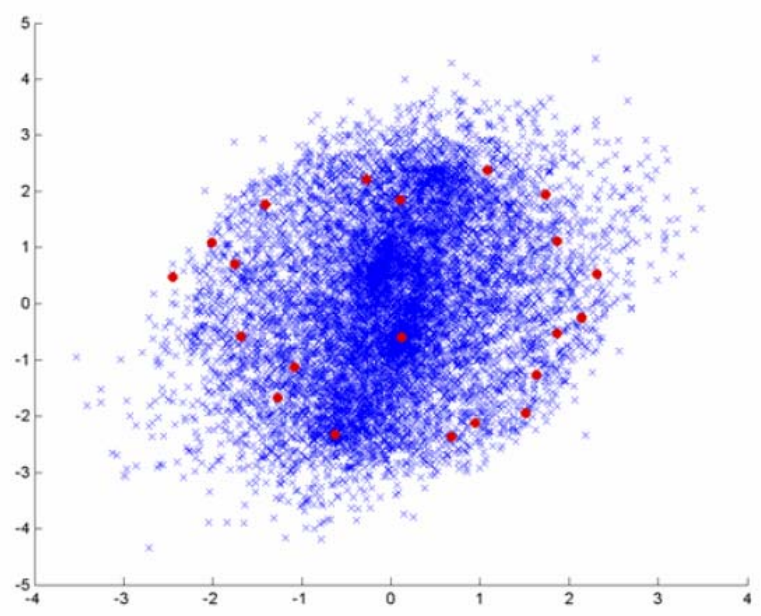

(c) 20 points + median point

Figure 5.3 - 10,000 gradual deformation samples 
We want to find a subset of 20 points that maximize the objective function given in Eq. 5.20. Running the algorithm produced the set shown in red in Figure 5.2. We want to use the 20 points as basis for GDM and examine the interpolation and extrapolation power when only this reduced subset is used. Using gradual deformation with Eq. 5.19, we drew 10,000 samples using the 20 points subset as basis models. The result in Figure 5.3a shows that most of the GDM sampling is at the boundary of the scatter cloud indicating a strong influence of the 20 points subset on sampling. We want GDM sampling to have better coverage of parameter space which requires adding interior points. Figure $5.3 \mathrm{~b}$ shows the effect of adding three interior points to the GDM basis models. The points have a strong attraction effect and may lead to less efficient sampling of extreme values. If we only add the median of the 100 original points to the subset, a more even sampling is achieved as shown in Figure 5.3c where a larger concentration of sampling is close to the median while at the same time achieving better sampling of extreme values. Figure 5.3c shows also that the GDM can extrapolate beyond the scatter cloud which improves the sampling efficiency. Figure 5.4 shows the plot of the objective function given by Eq. 5.20. An initial 20 points set were drawn randomly followed by the optimization loop were 100,000 swaps between subset and non subset points were performed. A total of 99 successful swaps were needed to maximize the objective function. The algorithm was optimized during iterations to calculate only the incremental difference resulting from a two-points swap to update the double summation given by Eq. 5.20 . 


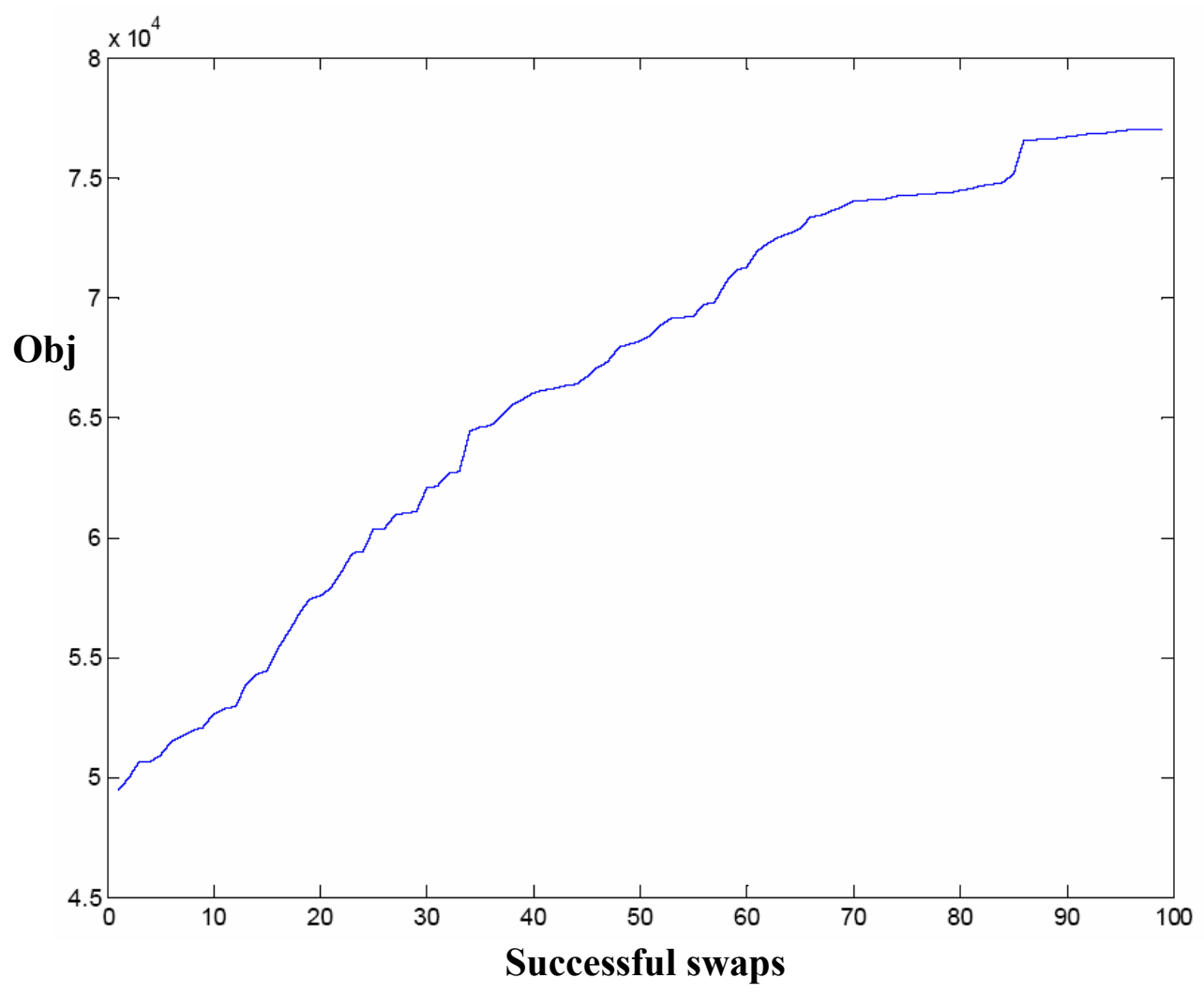

Figure 5.4 - Objective function maximization for optimum subset

\subsection{Approximate MCMC Workflow}

In the previous sections, we covered the mathematical formulation of the approximate MCMC. In this section we are going to outline the workflow we followed in applying the method. Figure 5.5 shows the flowchart diagram for approximate MCMC method with GDM sampling. The loop shown ends when a predefined number of samples are collected or a maximum number of iterations are reached. The main advantage and efficiency of approximate MCMC is experienced at the first conditional statement when potentially unacceptable proposals are filtered out using approximate acceptance check. Proposals that pass the approximate acceptance check are reevaluated using a full blown simulation run to calculate the exact likelihood similar to traditional MCMC. The effect is an accelerated convergence at a fraction of the cost of a traditional MCMC without 
sacrificing rigorousness. Both streamline and finite difference simulation can be used which cover a wide range of applications. Approximating the response using Eq. 5.4 requires few seconds even for large models which makes the method very efficient and practical for large field cases. Most importantly, the approximate response calculations do not require additional flow simulation. It is based on the response from the previously accepted state and the changes in parameter values proposed in the new state. In the next chapter, we will demonstrate the application of approximate MCMC using both streamline and finite difference simulators and compare the performance with respect to the traditional MCMC. 


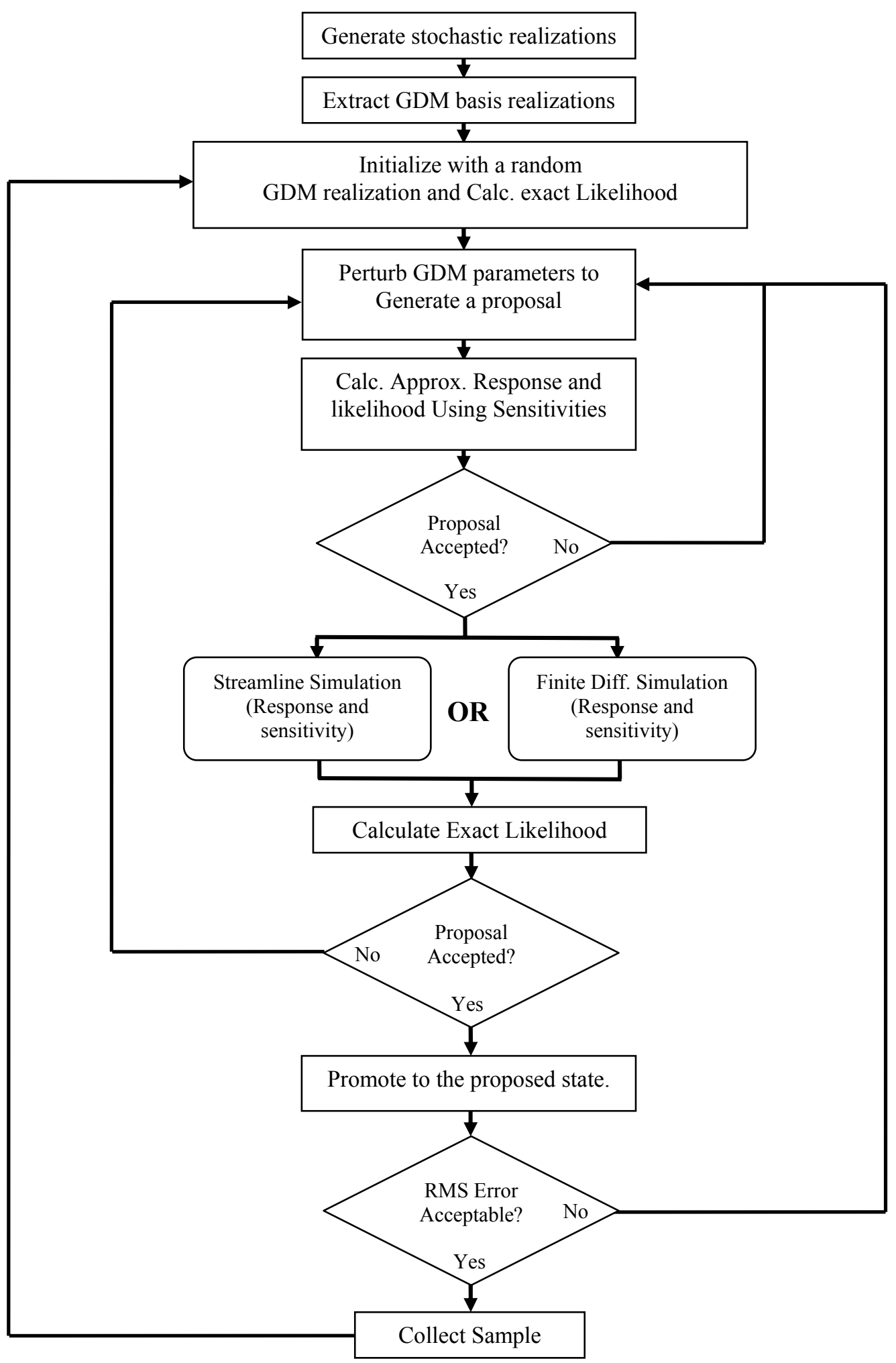

Figure 5.5 - Approximate MCMC flowchart 


\section{CHAPTER VI \\ APPROXIMATE MCMC: FIELD APPLICATIONS}

\subsection{Approximate and Full MCMC Performance Comparison}

A 2D mesh of $21 \times 21$ was used with a total of 441 grid cells to study the performance of approximate MCMC when compared with full MCMC. The reference model is shown in Figure 6.1a. The model exhibit complex connectivity patterns which will condition the movement of flood front across the model. We used gradual deformation for proposals generation by performing small perturbations to the deformation parameters. A total of 100 basis stochastic models were used for deformation. Figure $6.1 \mathrm{~b}$ shows the initial model used for both approximate and full MCMC. To compare the performance of approximate MCMC with full MCMC, we performed two runs where all parameters are fixed except for using the approximate Metropolis-Hastings acceptance check for the approximate MCMC. We used a standard deviation of $1 \%$ to calculate the covariance matrix, $C_{d}$, of water cut data in the objective function

$$
\psi^{0}=\left(d_{r}-g\left(m^{0}\right)\right)^{T} C_{d}^{-1}\left(d_{r}-g\left(m^{0}\right)\right)
$$




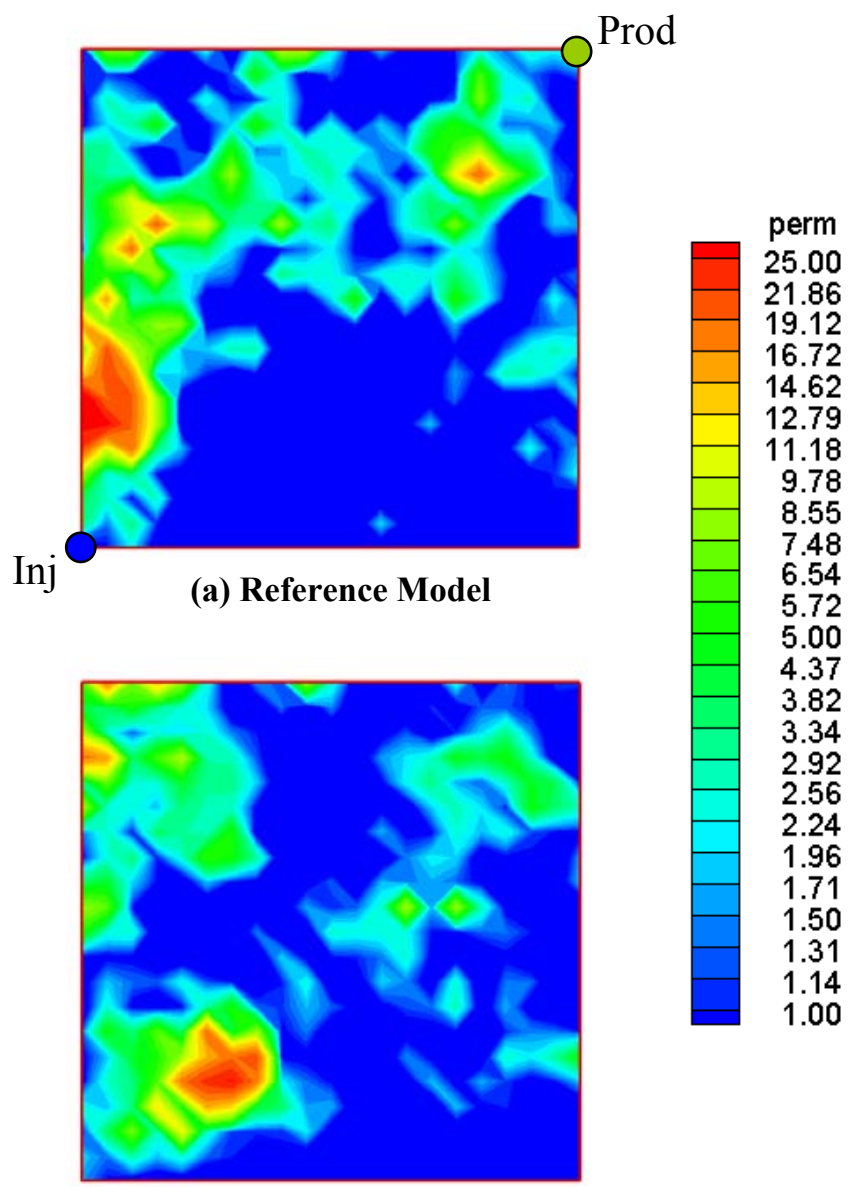

(b) Initial Model

Figure 6.1 - 2D case: Reference and initial model

Note that we did not include the prior term in the objective function since all proposals are conditioned to model covariance by design which is one of the advantages of using the gradual deformation method. Figure 6.2 shows the objective function reduction for both runs as a function of accepted proposals. The advantage of approximate $\mathrm{MCMC}$ is obvious in accelerating the minimization of the objective function where the burn-in time has been reduced by more than $50 \%$. 


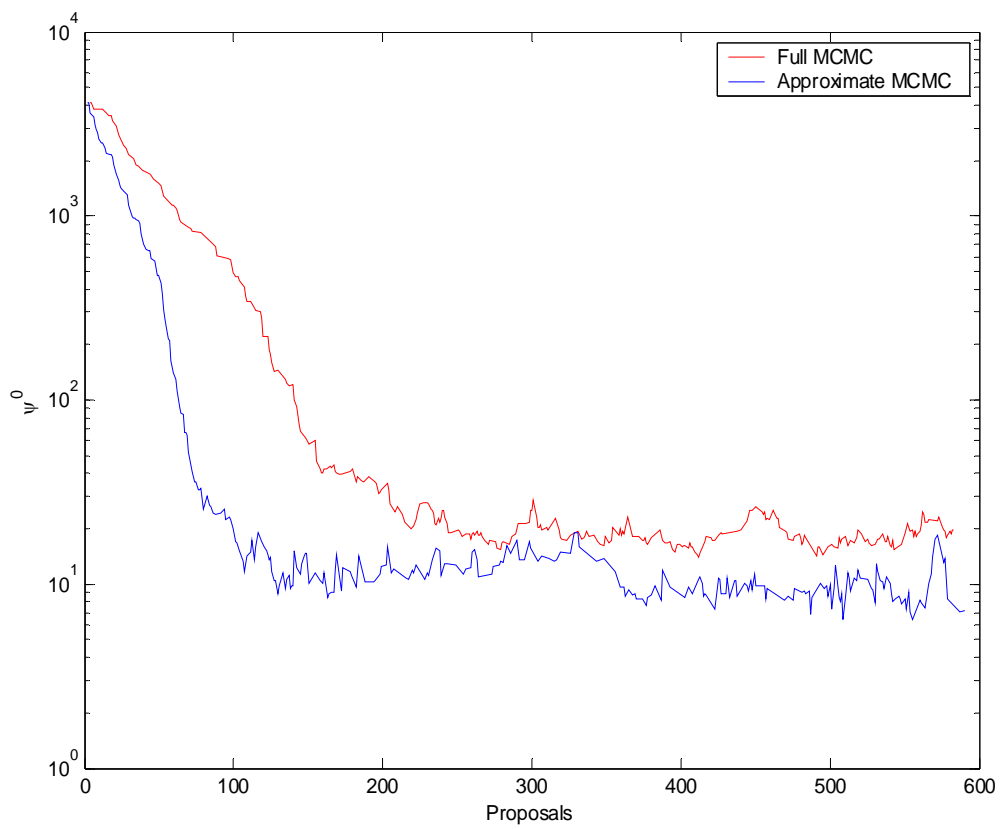

Figure 6.2 - Objective function reduction

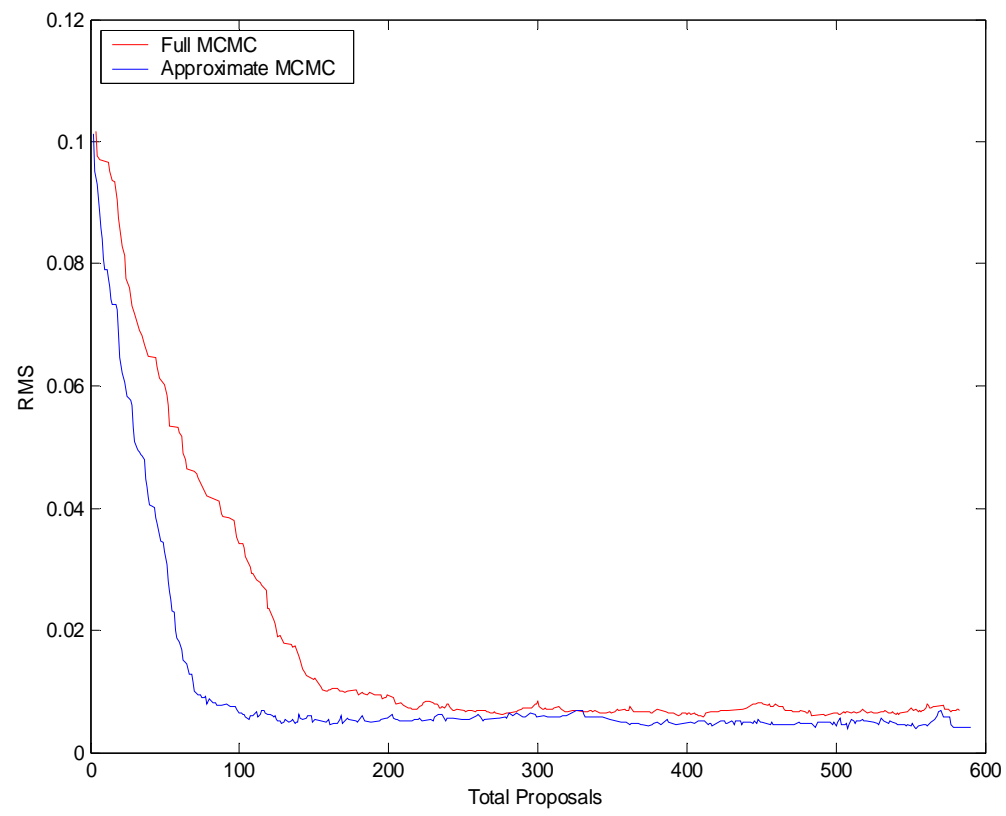

Figure 6.3 - RMS error reduction

The same effect is observed in RMS error reduction shown in Figure 6.3. Approximate MCMC will have the effect of filtering out the obvious rejections without paying the cost of running a full simulation. Such filtering does not ensure acceptance but 
will increase the probability of finding good proposals and thus, increasing MCMC efficiency.

Figure 6.4 shows the acceptance rate for both methods where total accepted proposals divided by total proposals. Approximate MCMC exhibit acceptance rate above $90 \%$ initially while full MCMC fluctuates below 50\%. The decline in acceptance rate indicates that the approximation is more accurate in filtering out rejections during burn-in time when the acceptance criteria ratio calculated by Eq. 5.1 has a high contrast between accepted and rejected proposals.

Figure 6.5 shows the final water cut match for the approximate MCMC. The full MCMC has the same initial WC and almost identical final match so it was not shown in the plot.

The results show the advantage of using approximate MCMC in accelerating the process and lowering the cost of MCMC.

Figure 6.6a shows convergence of permeability values in selected grid cells after burn-in time. The algorithm converged after approximately 300 iterations. Figure 6.6b shows convergence of some GDM parameters which were initialized with the same value. Samples are collected after parameters convergence to ensure that values are not biased by the initial values. 


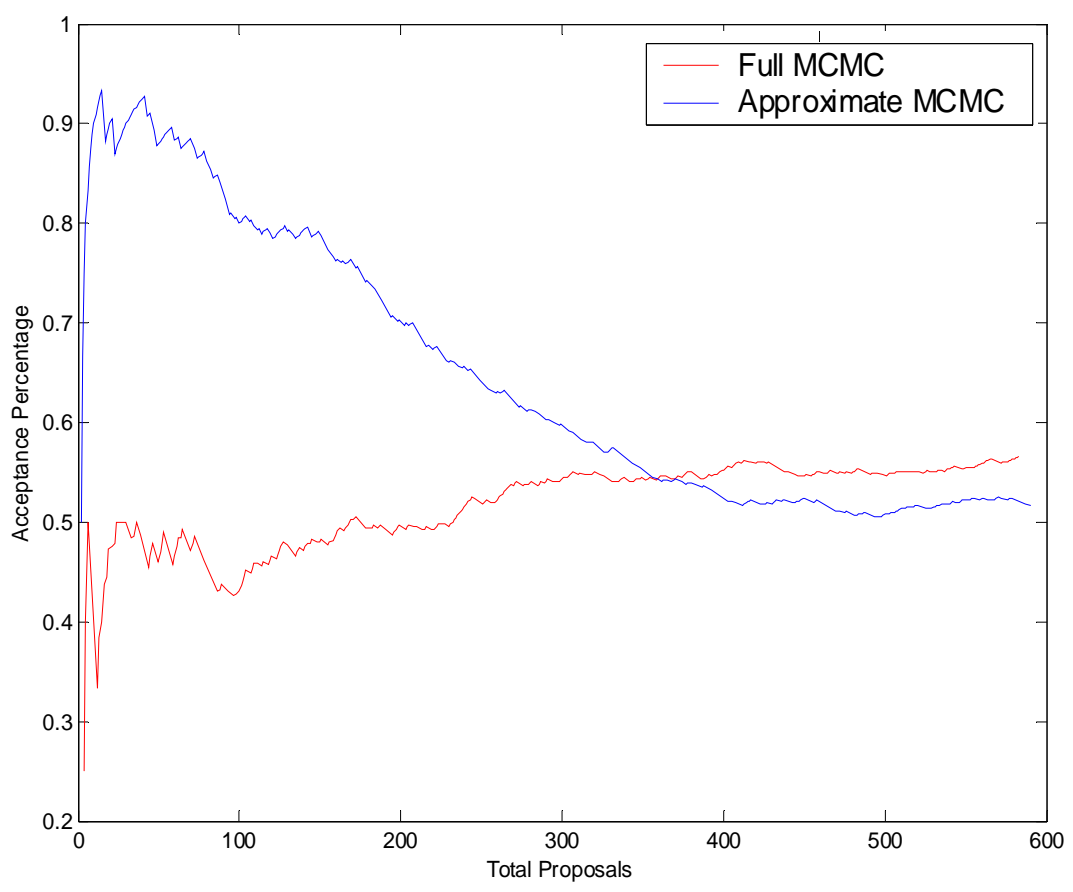

Figure 6.4 - Acceptance rate

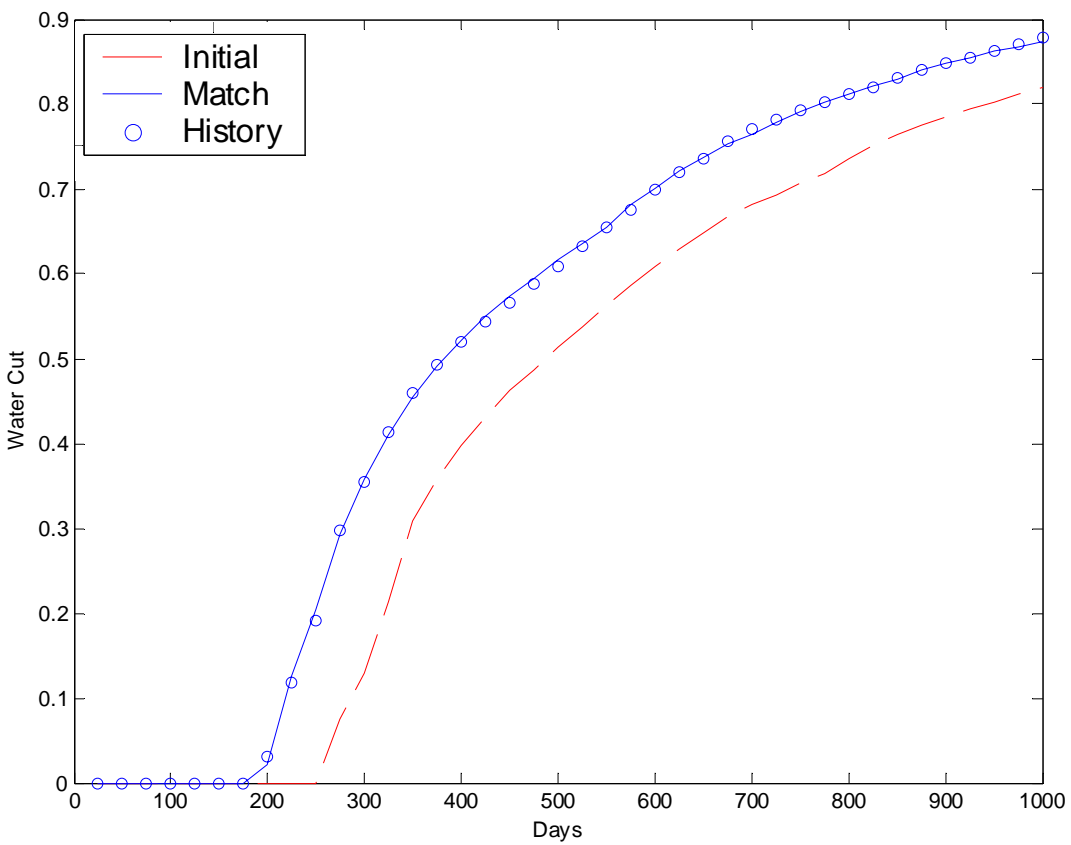

Figure 6.5 - Water cut match 


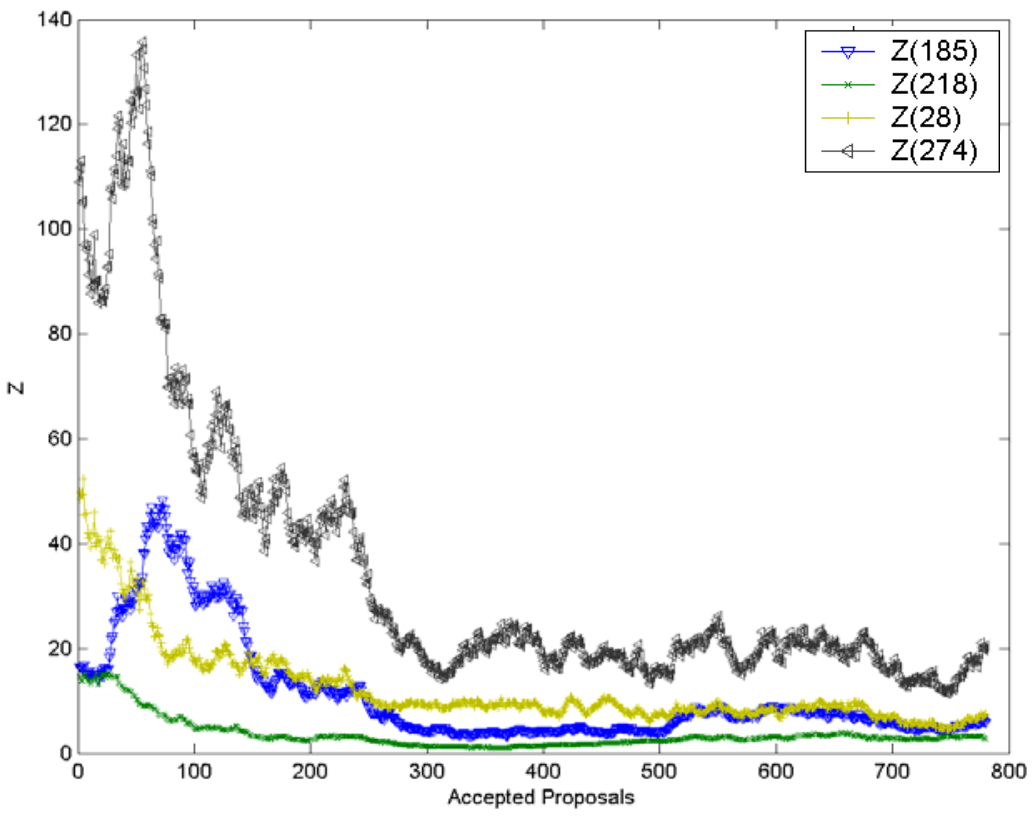

(a) Permeability convergence

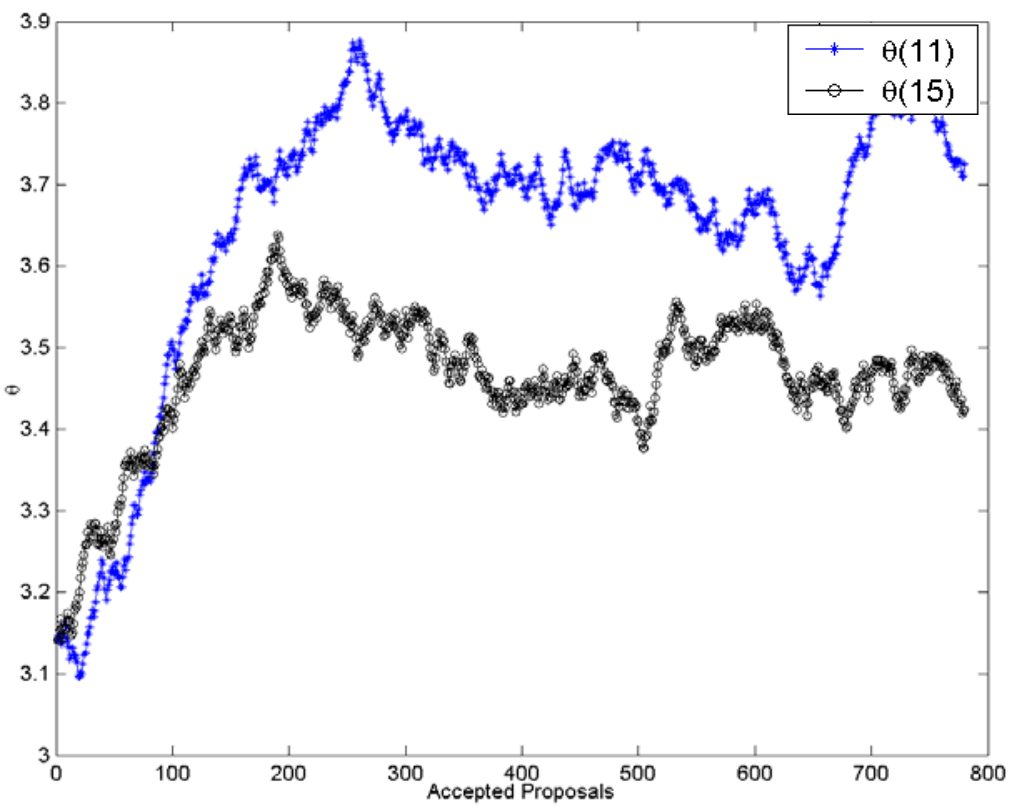

(b) GDM parameters convergence

Figure 6.6 - Convergence of parameters for approximate MCMC 


\subsection{D Example : Quarter 5-spot Waterflood}

In order to examine the sampling efficiency of approximate MCMC, we are going to use a quarter 5-spot model similar to the one used in section 6.1. Figure 6.7 shows the reference $21 \times 21$ model used. The problem is symmetric and the symmetry line is shown as a dashed line in Figure 6.7. Due to this symmetry, a model and its mirror image will give the same response leading to a bimodal distribution of permeability.

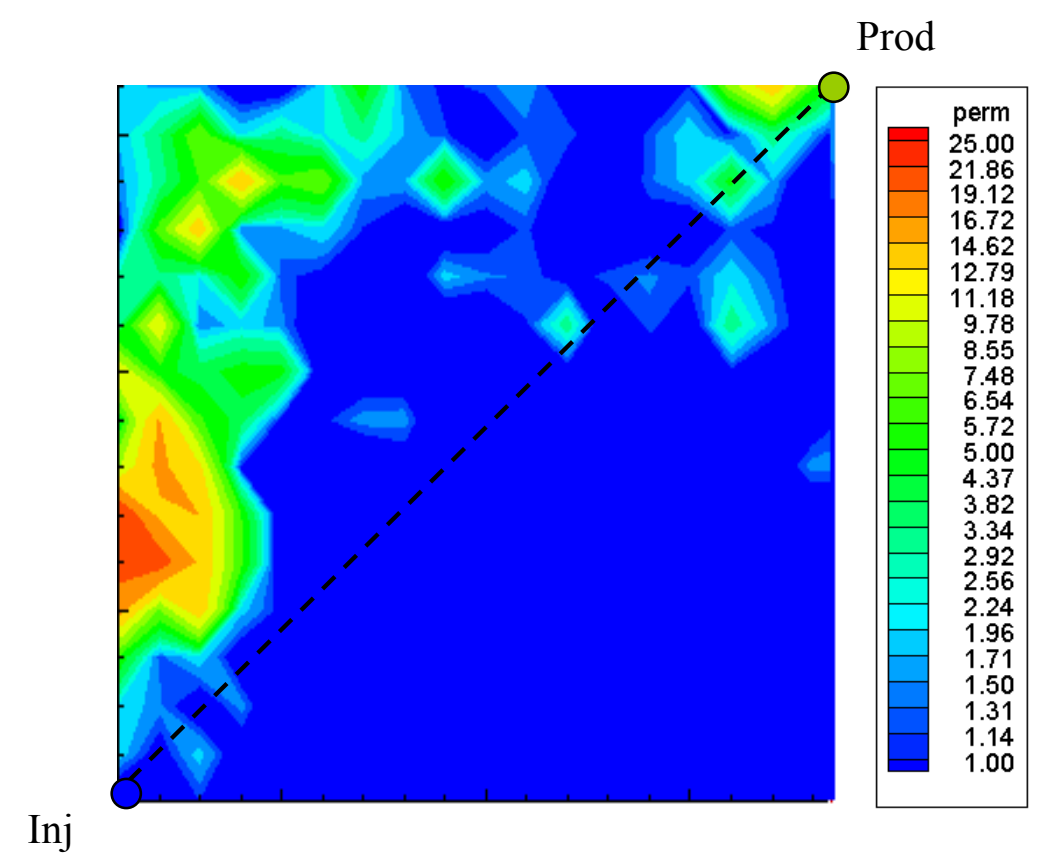

Figure 6.7 - 2D case: Reference model

We applied the approximate MCMC as outlined in the flowchart in Figure 5.5 with a streamline simulator. 400 stochastic models were generated using Sequential Gaussian Simulation (SGSIM). 30 basis realizations where extracted for GDM using the optimum basis selection algorithm outlined in section 5.2.4. The reference model was created separately and was not part of the 400 initial models dataset. A total of 150 outer iterations were executed and a maximum of 300 inner iterations were allowed before rejecting the initial proposal. The samples were accepted if the RMS error is reduced below 0.02 . A low standard deviation of $1 \%$ was used for water cut data. Figure 6.8 
shows the reduction of the objective function for one of the samples as a function of proposals. Out of the 150 iterations, 59 samples were collected or a $39.3 \%$ of the total.

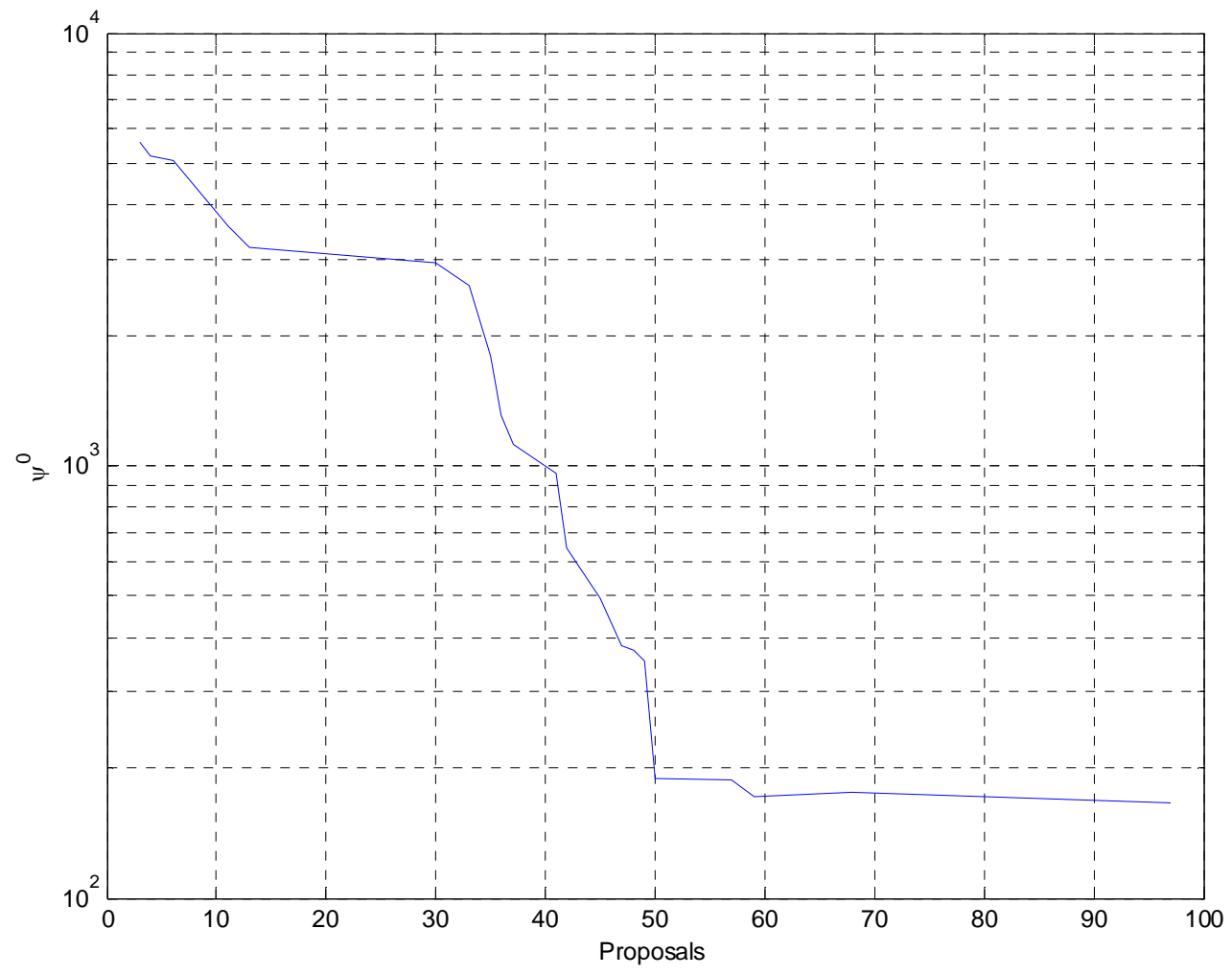

Figure 6.8 - 2D case: Objective function reduction 


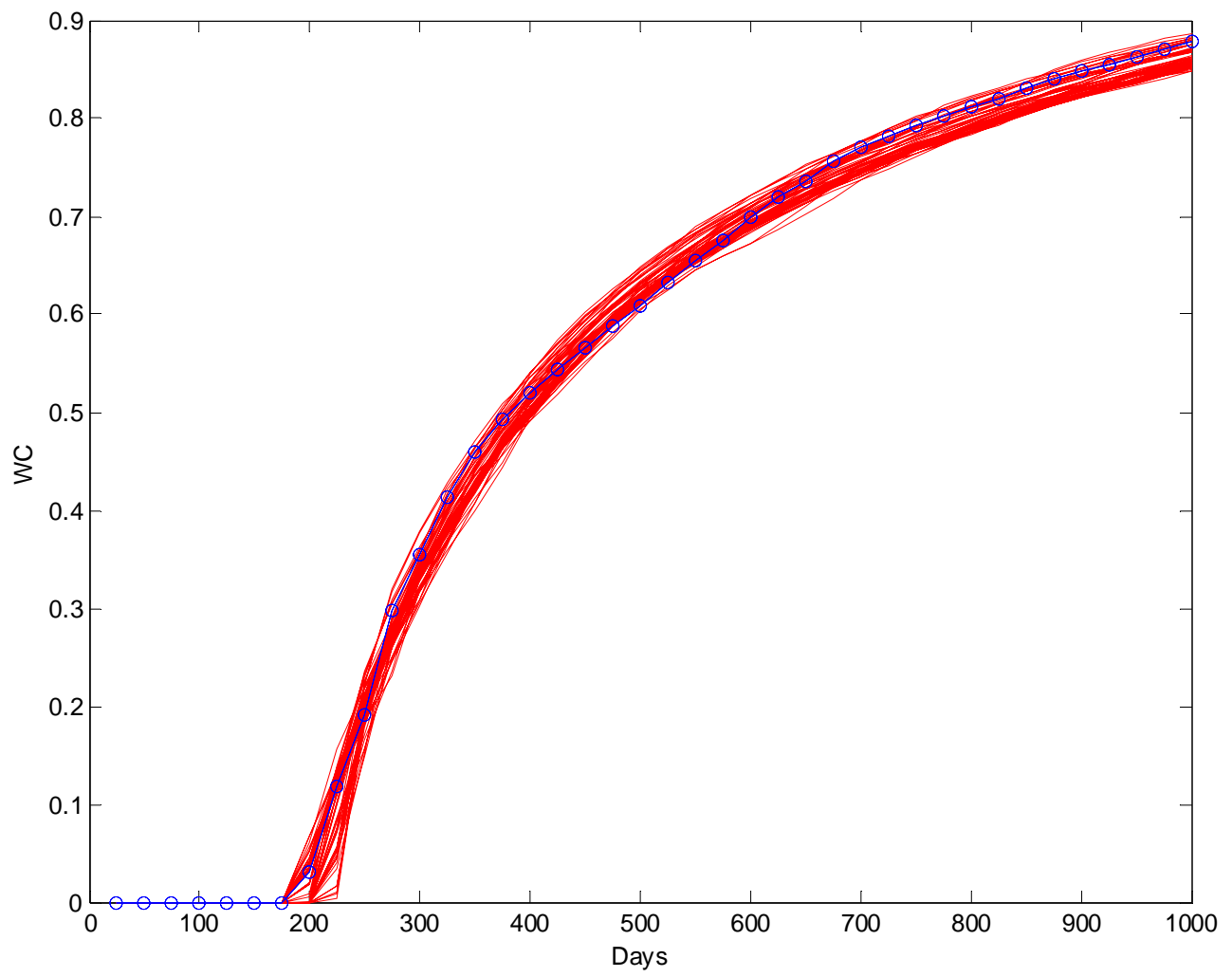

Figure 6.9 - 2D case: Final water cut match 

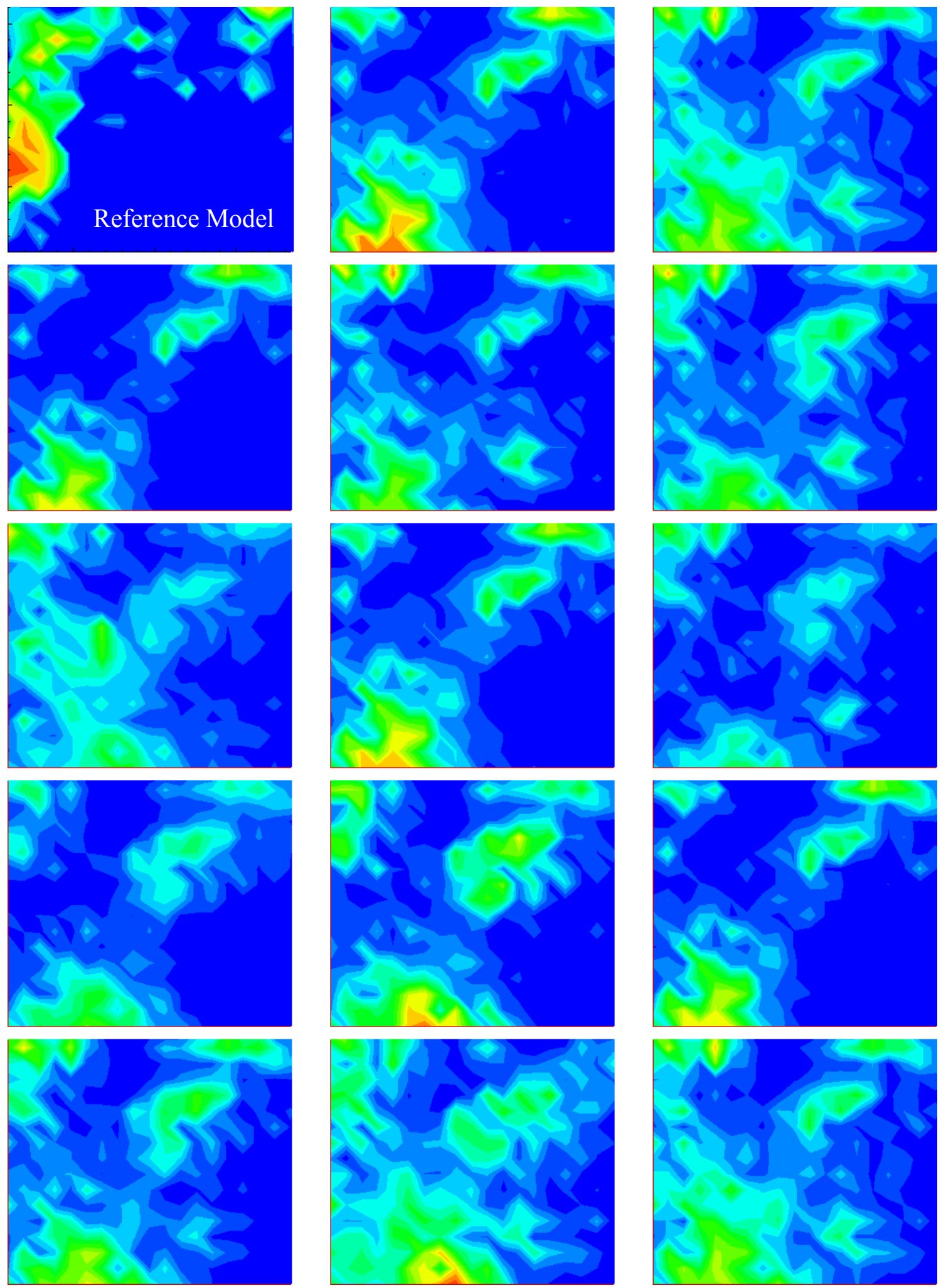

Figure 6.10 - 2D case: Selected samples 
Figure 6.9 shows the final water cut distribution for all 59 samples with the reference response. The narrow distribution is due to the low RMS error used as stopping criteria. A total of 14 selected samples are shown in Figure 6.10 indicating a wide range of possible permeability distributions and showing the uncertainty range. All these models honor static data, share the same covariance model and conditioned to observed dynamic data. Risk analysis can be performed using these models to bracket the uncertainty associated with reservoir response forecasting.

Figure 6.11 shows mean and variance for both the 30 GDM basis models used for sample proposals and the 59 collected samples. The effect of conditioning models to dynamic data is to reduce the samples variance since the additional information carried by the dynamic data narrows the range of permeability uncertainty as shown in Figure $6.11 \mathrm{~b}$ and Figure $6.11 \mathrm{~d}$. The process is computationally efficient and can be performed on a desktop PC due to the low memory requirements for calculating and storing analytical sensitivities. We used a fast streamline simulator for this particular 2D case and the total time needed to perform 150 iterations was 6.7 hours on AMD 3.2+ 64bit machine. 


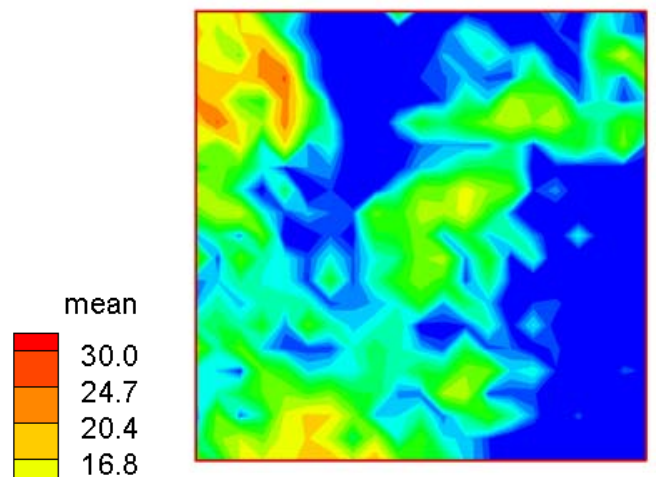

(a) 59 Samples mean

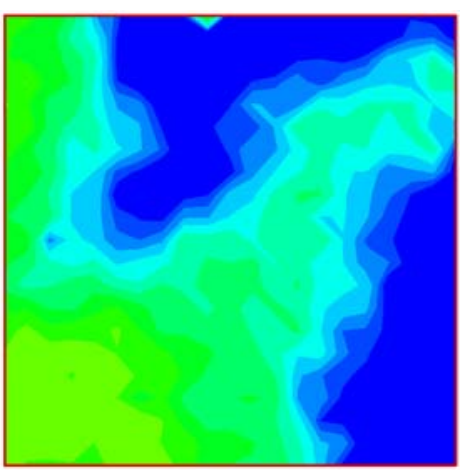

(c) Basis models mean

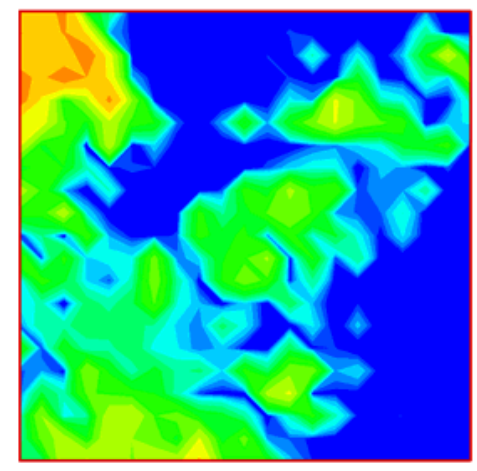

(b) 59 Samples variance

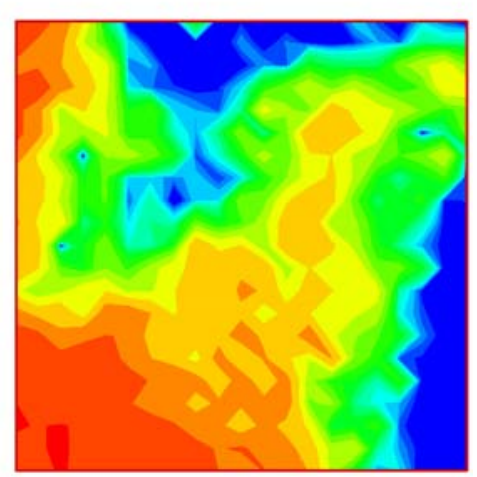

(d) Basis models variance

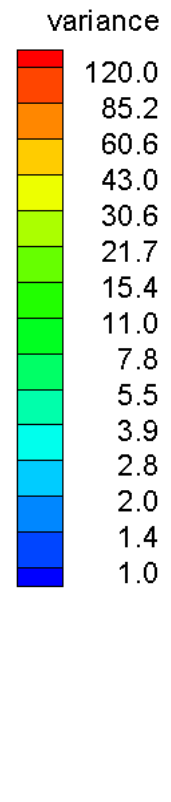

Figure 6.11 - 2D case: Mean and variance of samples 


\subsection{D Example: Goldsmith Field Case}

The 3D example is for the Goldsmith field case, a carbonate West Texas reservoir.

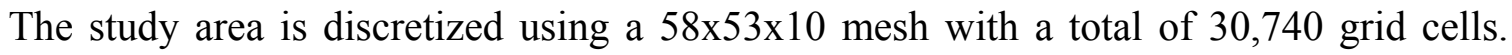
There are nine inverted 5-spot patterns covering 320 acres with average thickness of 100 $\mathrm{ft}$. The model has 11 injectors and 31 producers but only 9 producers showed significant water cut production in the first 20 years of waterflooding and will be used for water cut match. Figure 6.12 shows the well configuration of the study area and Figure 6.13 shows well schedule with infill and conversions.

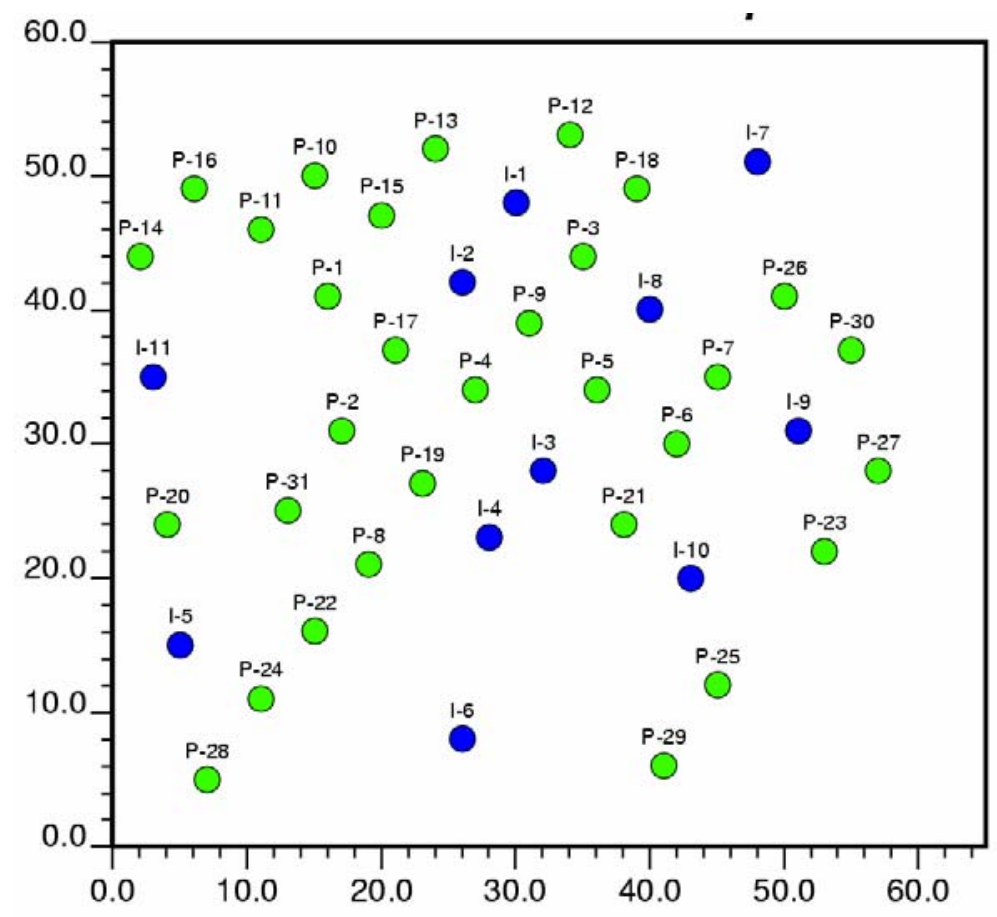

Figure 6.12 - 3D case: Well configuration 
Collocated sequential Gaussian simulation was used to stochastically generate 200 permeability realizations collocated with a prior porosity model. A total of 100 realizations were retained using the GDM basis optimization program. Figure 6.14 shows the prior porosity model and three basis permeability realizations used for GDM.

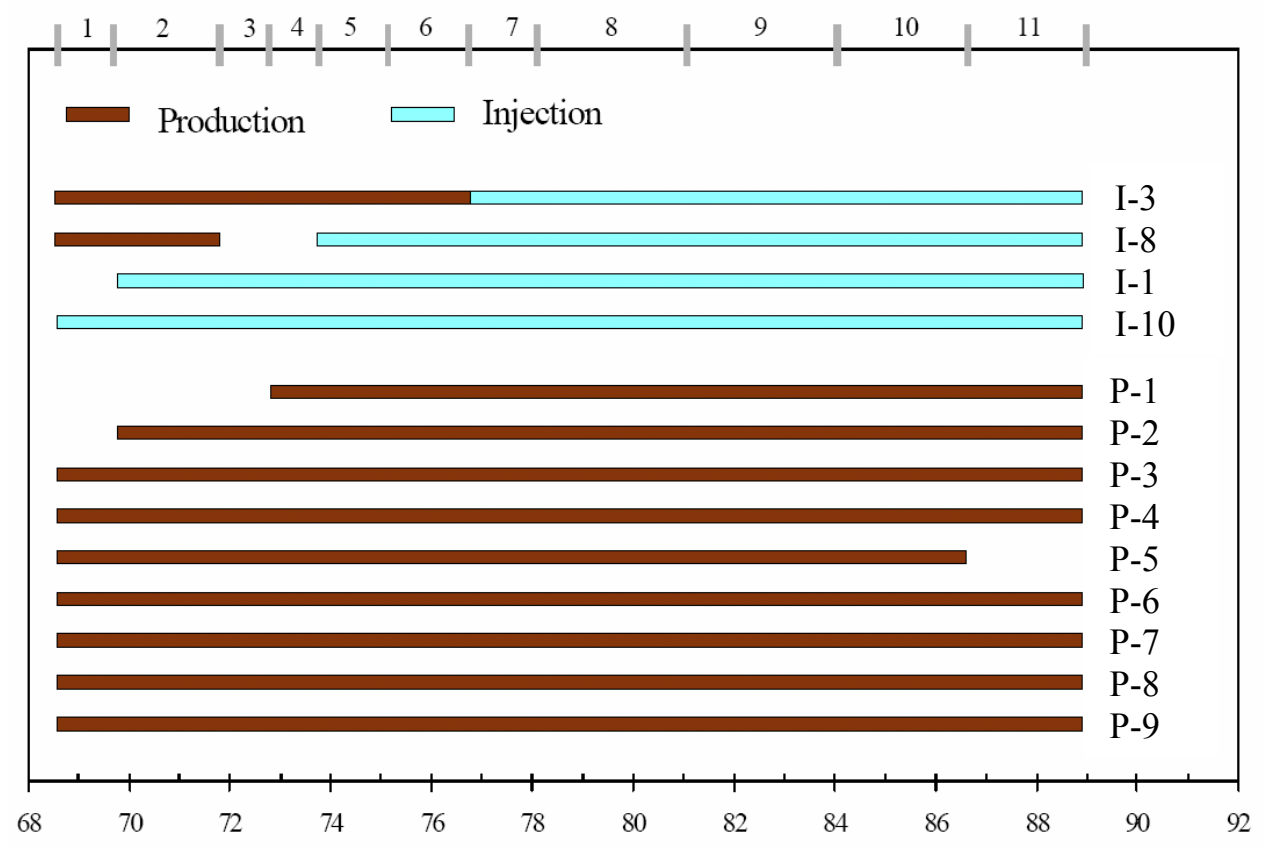

Figure 6.13 - 3D case: Well schedule 

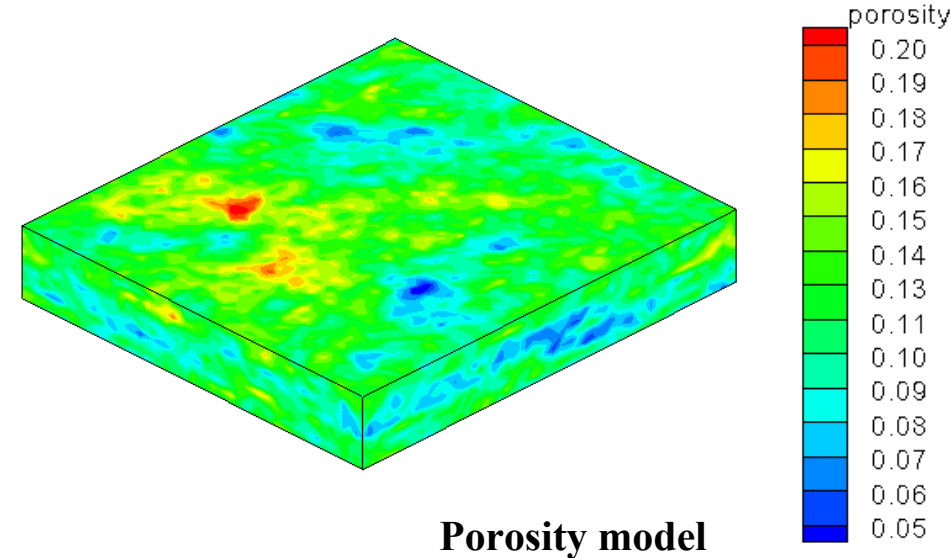

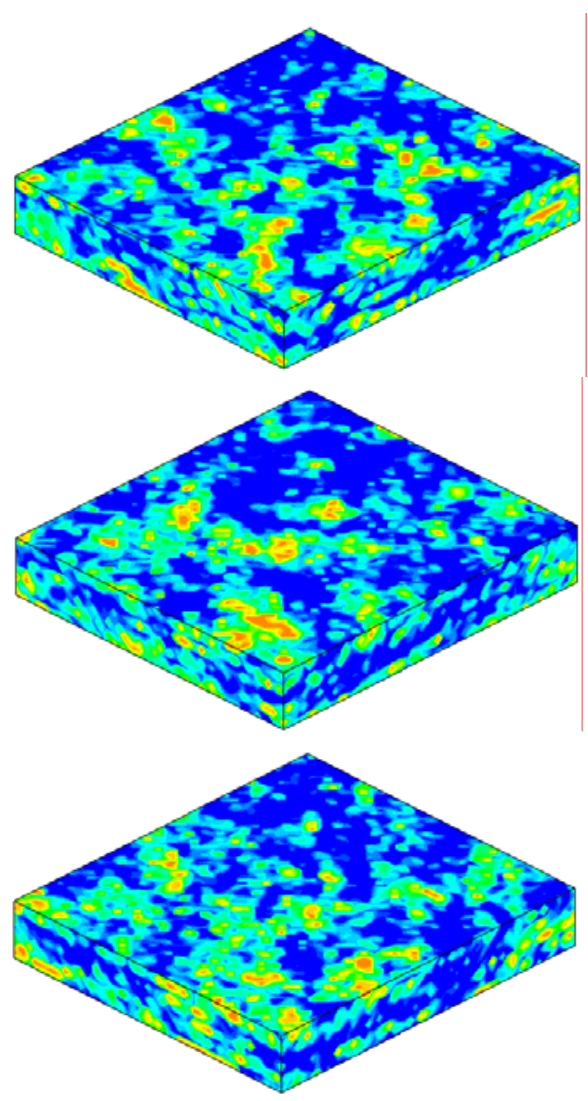

Three stochastic permeability models

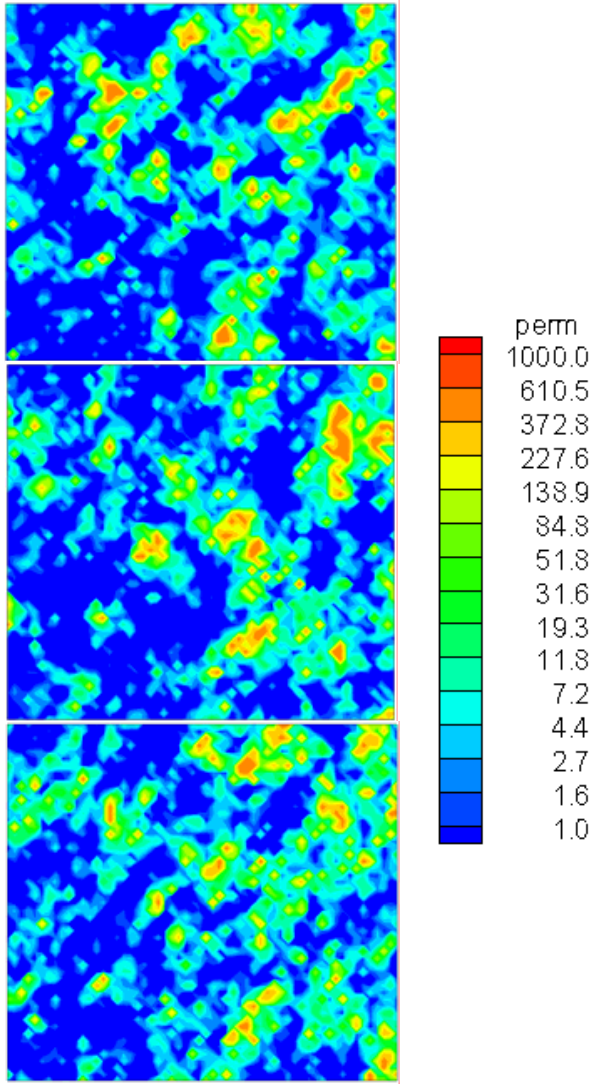

Layer \# 2

Figure 6.14 - 3D Case: Porosity and permeability models 
The 100 basis models exhibit a wide range of variability and connectivity patterns which will enable the GDM to generate proposals with a wide range of permeability distributions. Figure 6.15 shows four samples generated by GDM using random deformation parameters. The models range from the highly heterogeneous to the relatively smooth and from the highly connected to the patchy. All the models, however, honor the same static data and can be gradually deformed to a new state using a small perturbation to the GDM parameters. The covariance function was not preserved since the models are conditioned to static data, and hence dependent. We, however, assumed that that covariance structure is unknown and hence, contributes to the underlying uncertainty. The wide range of structures generated by using this approach increased the sampling efficiency while still honoring static data. This approach does not strictly follow the GDM method but it proved to be more efficient in sampling the parameter space by allowing the covariance structure to vary.

We ran approximate $\mathrm{MCMC}$ using the commercial finite difference simulator Eclipse. A maximum limit of 150 total runs, including approximate and full simulation runs, per inner iterations was used and the target RMS error was set to 2.7. A total of 17 outer iterations were performed in 10 days on a dual Xeon 3.06 PC and 13 samples were 

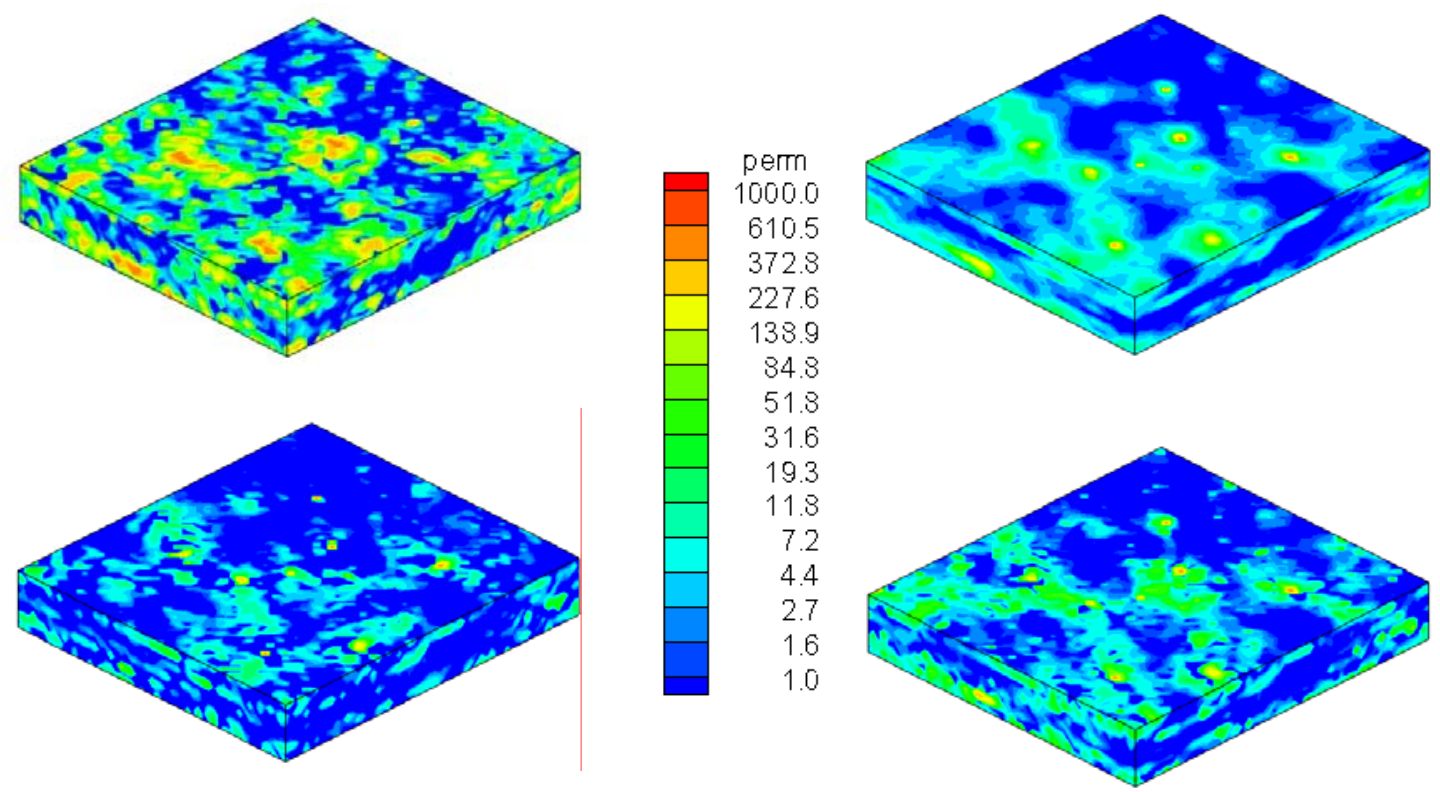

Figure 6.15 - 3D case: GDM samples 
collected. Figure 6.16 show the RMS error reduction as a function of full simulation runs performed for the 13 samples. The relatively fast drop in RMS is due to the increased acceptance rate as obvious rejections are filtered out during the approximate likelihood calculation.

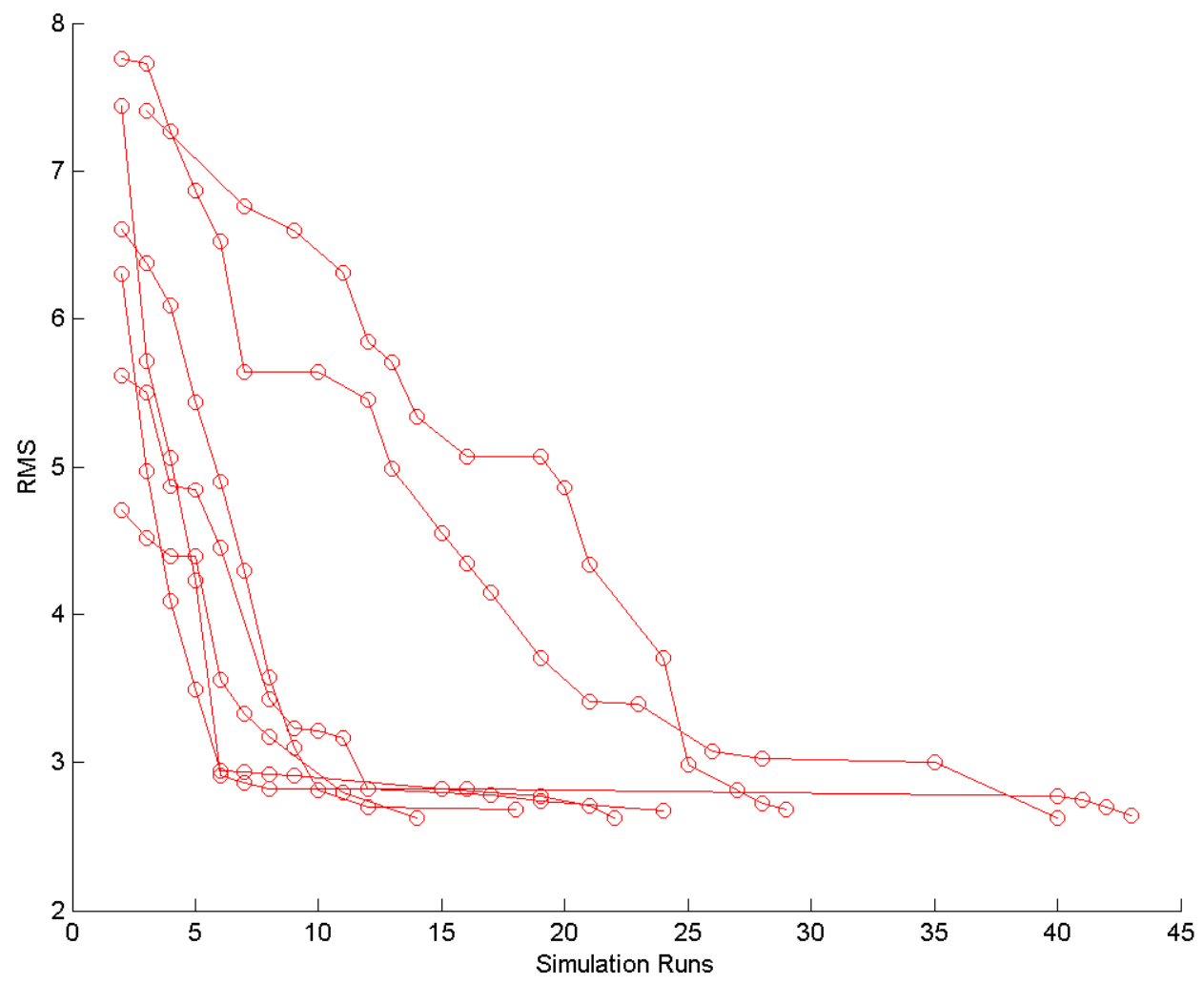

Figure 6.16 - RMS reduction 
The improved acceptance rate can be seen clearly in Figure 6.17 where we ran both approximate and full MCMC using the same initial model. Figure 6.17 shows the acceptance rate as the ratio of accepted proposals over total proposals. Approximate MCMC maintained a high acceptance rate during the 40 simulations runs needed to converge to an RMS error of 2.7. Full MCMC, however, required 110 simulation runs to lower the RMS to the same level. The speed up gained by approximate MCMC will lead to a shorter burn-in time and a higher convergence rate.

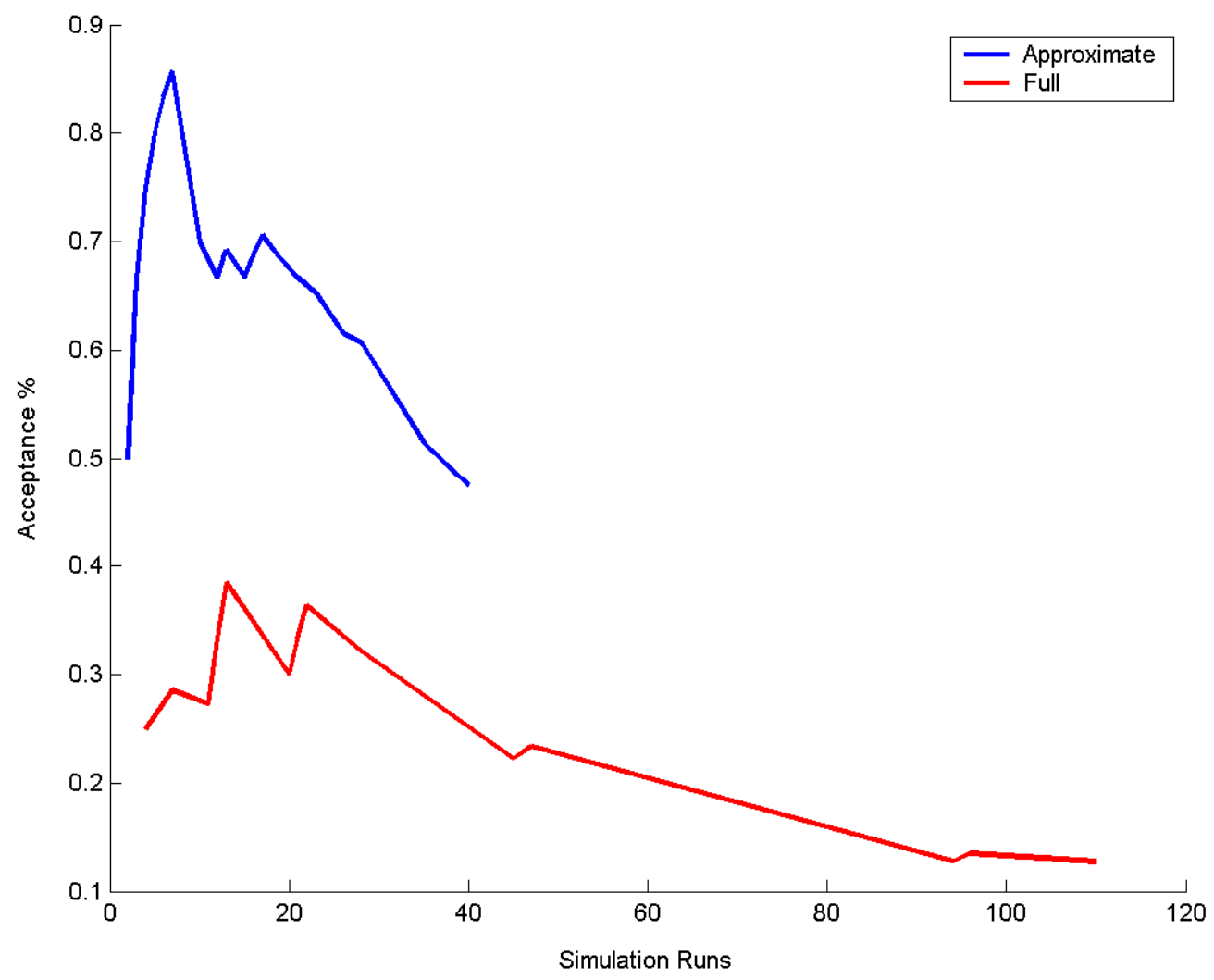

Figure 6.17 - Acceptance rate for full and approximate MCMC 
It should be noted that the total number of iterations is comparable for both cases if we consider the approximate evaluations which requires only few seconds to calculate. Figure 6.18 shows a comparison between full and approximate MCMC RMS error

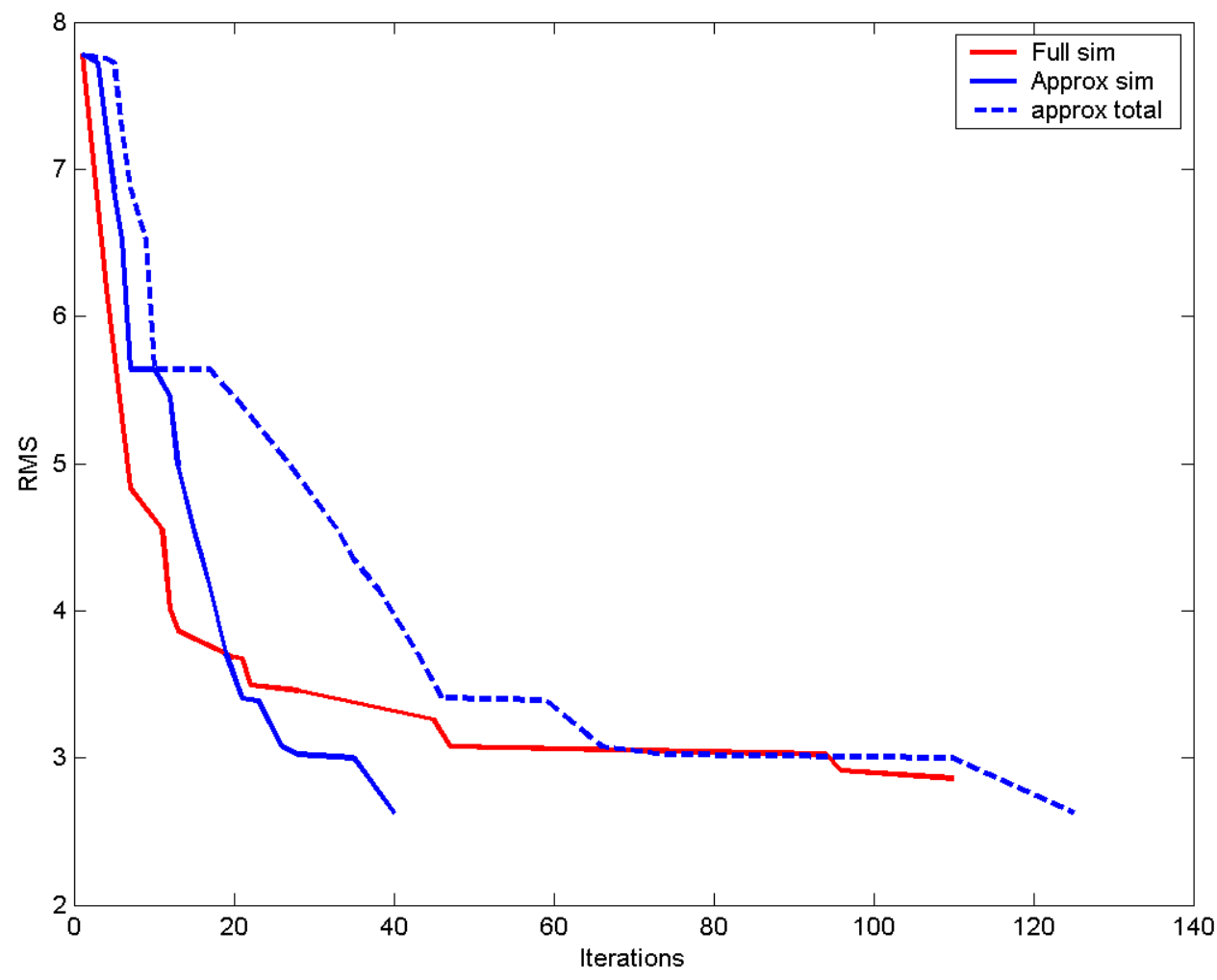

Figure 6.18 $-1^{\text {st }}$ sample: RMS reduction for full and approximate MCMC 
reduction for the first sample collected. When considering the total number of iterations, both methods evaluated a comparable number of proposals. The approximate MCMC, however, required only 40 simulation runs compared to 110 for the full MCMC which translates to $64 \%$ reduction in computational cost for this particular run. Figure 6.19 shows three samples collected using approximate MCMC with the corresponding RMS reduction. As expected, the integration of dynamic data reduced the variability considerably while still maintaining the geologic realism. All the samples collected honor static data at their location by design while still being conditioned to the observed dynamic data.

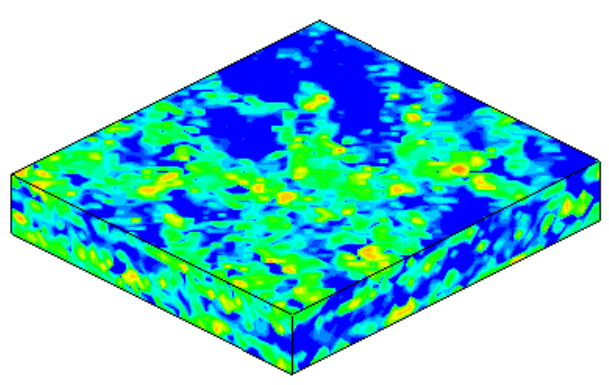

Samples\#1

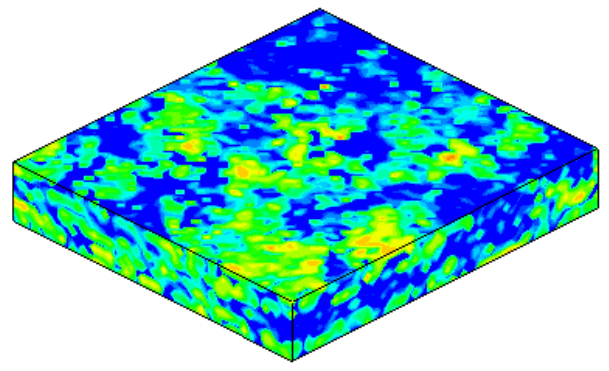

Samples\#6
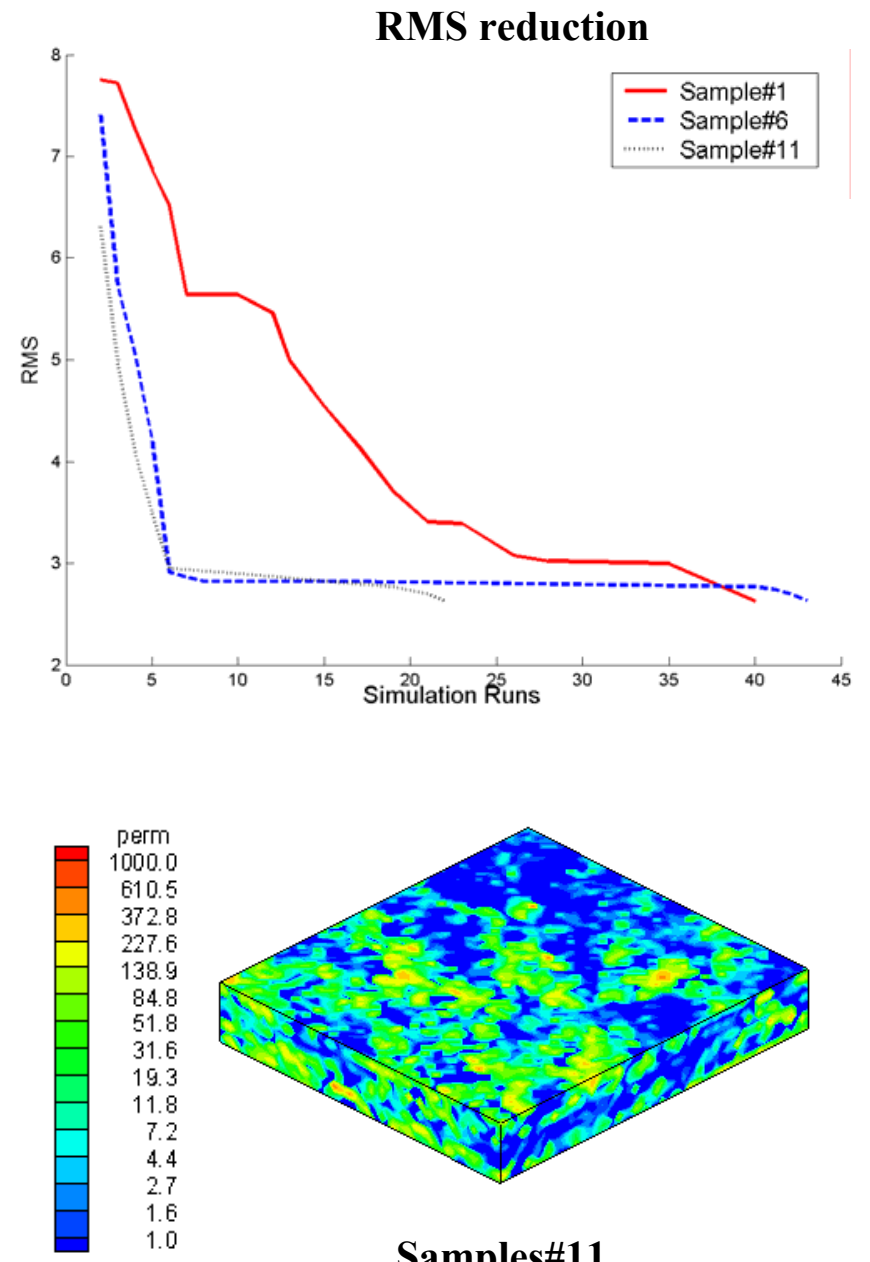

Samples\#11

Figure 6.19 - Approximate MCMC: Three collected samples 
The integration of dynamic data should reduce the uncertainty observed in unconditioned models and produce samples that reflect the true uncertainty. In Figure 6.20 , variance for five selected layers is shown for both the GDM basis models and the approximate MCMC samples. The reduction in uncertainty is clear where the variance has been reduced significantly when compared to the original basis models that were used to generate proposals.

The reduction in uncertainty is also reflected in samples water cut response as shown in Figure 6.21. The initial water cut responses shown in Figure 6.21a are for the initial 13 realizations that are conditioned to static data. The final match shown in Figure $6.21 \mathrm{~b}$ for the approximate MCMC samples shows the reduction in reservoir response uncertainty 


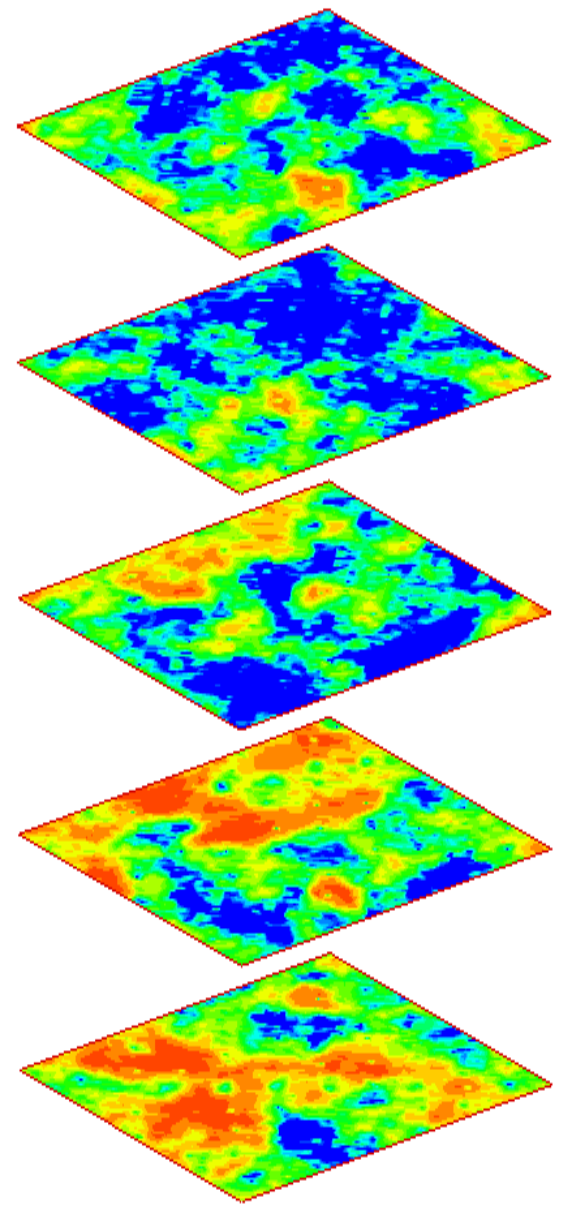

(a) Basis realizations

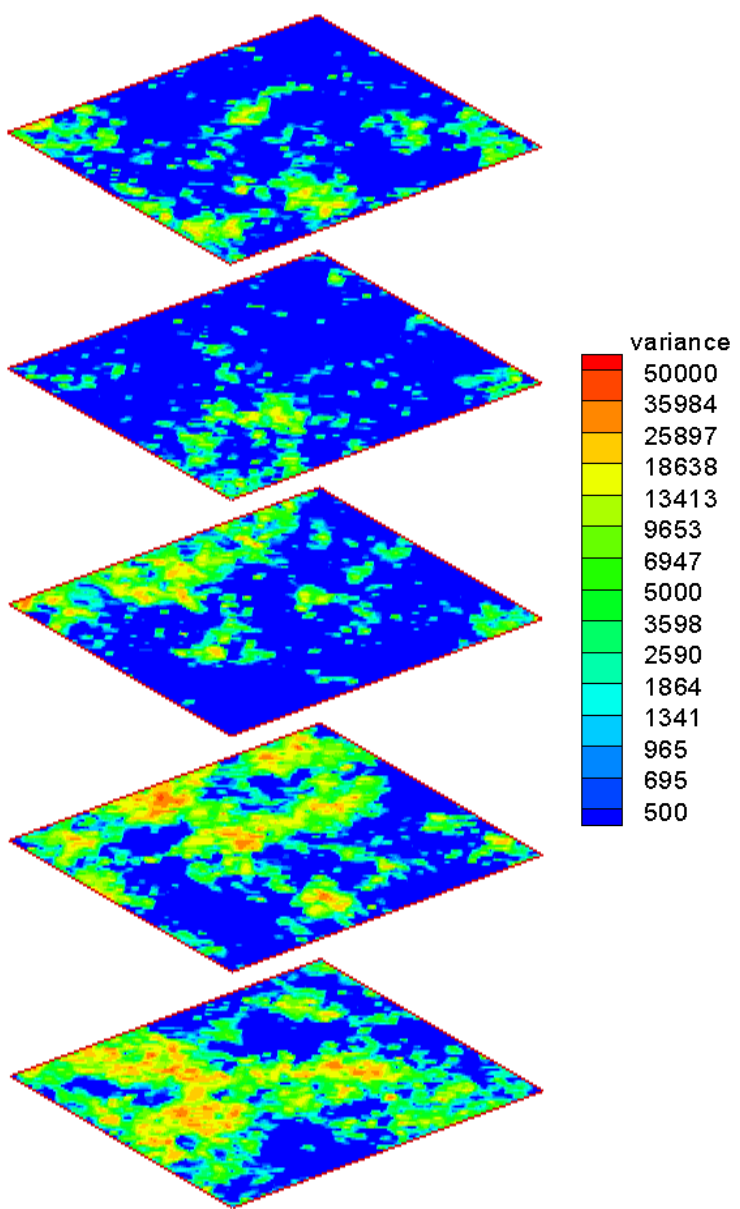

(b) Approximate MCMC samples

Figure 6.20 - Permeability variance reduction 

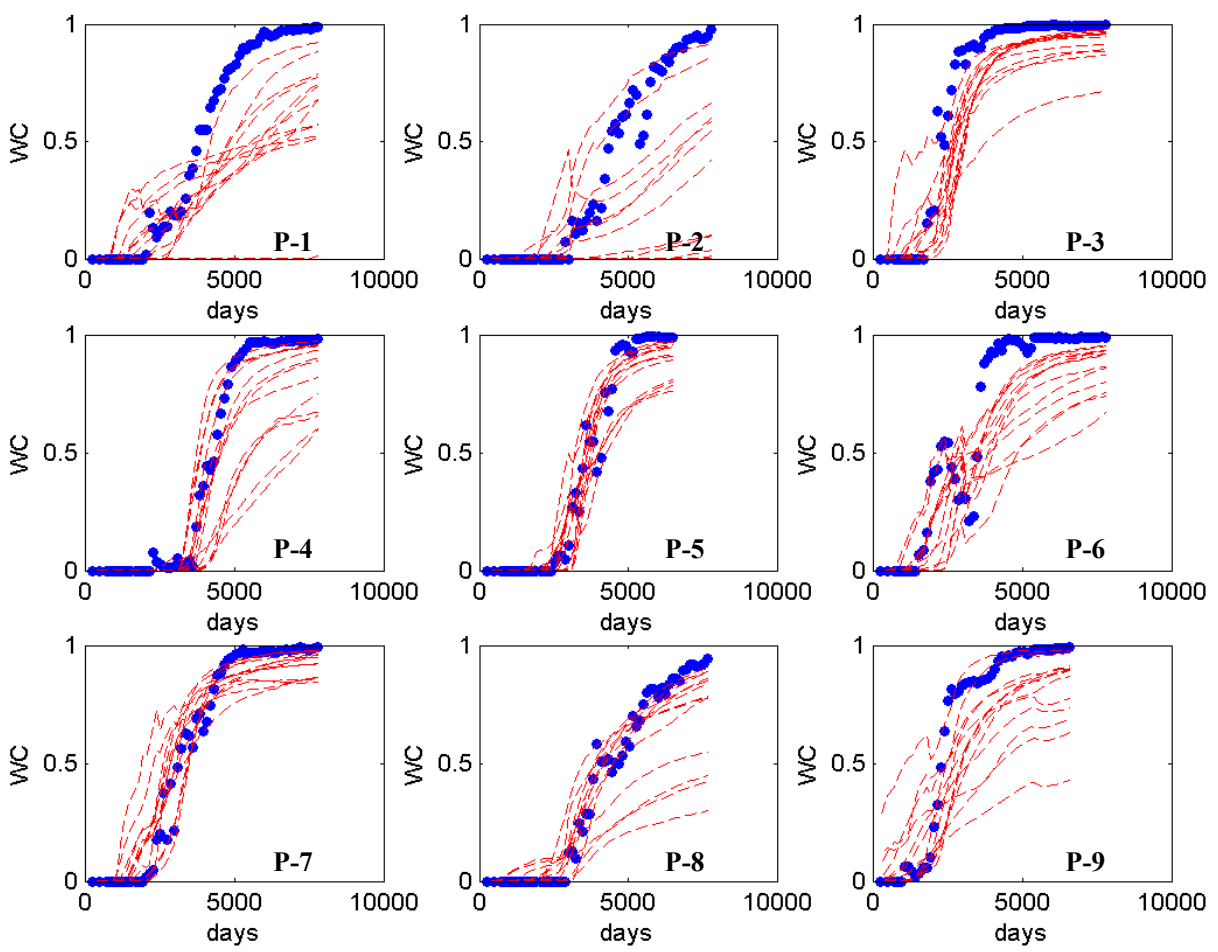

(a) Initial match
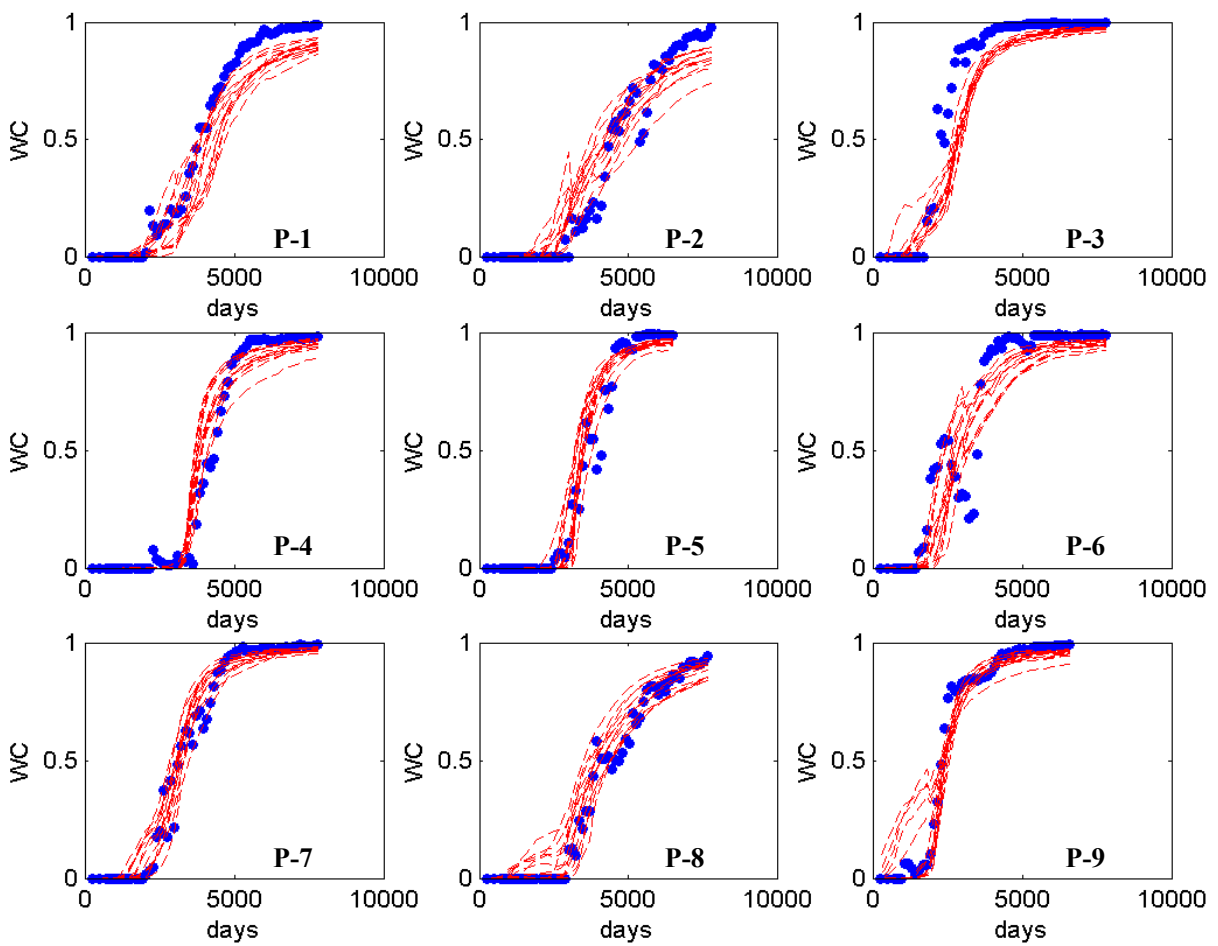

(b) Final match

Figure 6.21 - Initial and final water cut response 


\section{CHAPTER VII \\ GENERALIZED TRAVEL TIME INVERSION USING GRADUAL DEFORMATON METHOD}

\subsection{Introduction}

Integration of dynamic data into high resolution geological models is performed through an inverse problem. Specifically, we attempt to minimize an objective function that quantifies the deviation of a model response from an observed response. This is done by perturbing reservoir parameters such as permeability and porosity and using a data misfit measure such as travel time or response amplitude error. There are many methods available in literature to handle such problems and they are generally fall into three categories; randomized, enumerative and gradient based methods. Randomized methods such as simulated annealing ${ }^{57}$ and genetic algorithms ${ }^{58}$ attempt to find the global minimum by integrating a random process into the workflow to avoid getting trapped in localized minima. While these methods are theoretically capable of reaching the global minimum, they are generally slow and can be extremely expensive and inefficient for high resolution models. Enumerative methods calculate the objective function over a discredited parameter space and retain the sample associated with the minimum. For large problems with many parameters, such methods can be the least efficient and the most expensive if not intractable. Gradient based methods are localized methods where a local minimum is sought in the vicinity of a prior model. The use of streamline-based analytical gradients ${ }^{6-8}$, which requires a single simulation run to obtain, has made such methods extremely practical for history matching high resolution geological models. Practical applications of streamline-based gradient methods require using additional constraints to regularize the solution and preserve the geological structures obtained from static data. In the deterministic approach ${ }^{8}$, a norm constraint is used to insure that the final match is close to the prior model which incorporates all the static data, conceptual 
framework and seismic data. A roughness constraint is also added to control model smoothness which regularize the solution and account for the fact that inversion is more appropriate in retrieving large scale structures rather than small scale fluctuations.

Gradient based methods attempt to match observed response by performing travel time, amplitude or generalized travel time shift. ${ }^{8}$ It has been shown ${ }^{6,59-60}$ that travel time inversion possess quasilinear properties making it more robust and computationally efficient compared to amplitude inversion which can be highly non-linear. This leads to a rapid convergence even if the prior model is not close to the final match. Moreover, sensitivities between wells are more uniform in travel time inversion while amplitude sensitivities are more localized around wells. Such localization of sensitivities leads to over-correction around wells. The Generalized Travel Time inversion (GTT) improves upon travel time inversion by reducing the problem into minimizing a single parameter per well, the generalized travel time misfit. ${ }^{60}$ GTT retains the favorable characteristics of travel time inversion while reducing the size of the problem considerably. Studies have shown that GTT performs amplitude match close to the solution which explains its success in matching arrival times and response amplitude without the need to resorting to a two-step iterative matching. ${ }^{8}$

Optimization using the Gradual Deformation Method (GDM) has been investigated by several authors. ${ }^{54,61}$ The main advantage of GDM is that it generates models that are constrained to both spatial data and the covariance structure which helps in preserving geological structures and realism. While in its general formulation the method can be used to generate a deformed model from a large number of basis models, published studies were limited to deforming only two or three models at a time. This is mainly due to the use of numerical perturbation to calculate sensitivities which can be expensive for high resolution models. In this work, we will formulate the generalized travel time inversion using the gradual deformation method. The method will require a single simulation run to calculate the GTT sensitivities to the GDM parameters making it computationally efficient. Unlike other gradient methods, matched model will preserve the conditioning static data and spatial covariance structure while matching dynamic data at the same time. 


\subsection{Mathematical Formulation}

In section 5.2.3 we introduced the mathematical formulation for the GDM. A gradually deformed model can be calculated as a linear combination of $m+1$ basis models. Eq. 5.18 is used for any number of basis models.

$$
Y(x)-m_{Y}=\sum_{i=0}^{M} \alpha_{i}\left(Y_{i}(x)-m_{Y}\right)
$$

There are $m$ GDM parameters needed to calculate the $m+1$ weights, $\alpha_{i}$, in Eq. 5.18. The weights are calculated fro any number of parameters using Eq. 5.19

$$
\begin{aligned}
& \alpha_{0}=\prod_{i=1}^{m} \cos \left(t_{i}\right) \\
& \alpha_{i}=\sin \left(t_{i}\right) \prod_{j=i+1}^{m} \cos \left(t_{j}\right) \quad t \in(-\pi, \pi) \\
& \alpha_{m}=\sin \left(t_{m}\right)
\end{aligned}
$$

To calculate reservoir parameter sensitivity to GDM parameters, we need to combine Eqs. 5.18 and 5.19 and differentiate with respect to GDM parameters. The derivation is shown in Appendix A where the sensitivities to the kth GDM parameter is given by the following,

$$
\begin{aligned}
\frac{\partial Y}{\partial t_{k}}= & {\left[-\sin \left(t_{k}\right) \prod_{i=1}^{m} \cos \left(t_{i}\right)_{i \neq k}\right] Y_{0} } \\
& +\sum_{i=1}^{k-1}\left[-\sin \left(t_{k}\right) \sin \left(t_{i}\right) \prod_{j=i+1}^{m} \cos \left(t_{j}\right)_{j \neq k}\right] Y_{i} \\
& +\left[\cos \left(t_{k}\right) \prod_{j=k+1}^{m} \cos \left(t_{j}\right)\right] Y_{k}
\end{aligned}
$$

With reservoir parameters sensitivity to GDM parameters available, we can derive GTT sensitivities to GDM parameters using the chain rule, 


$$
\frac{\partial \Delta \tilde{t}}{\partial t}=\frac{\partial \Delta \tilde{t}}{\partial Y} \frac{\partial Y}{\partial t}
$$

where $\frac{\partial \Delta \tilde{t}}{\partial Y}$ is given by Eq. 3.40 .

We can use the deterministic formulation ${ }^{8}$ to solve the minimization problem. In the deterministic approach, we seek to minimize the following data misfit function

$$
O b j=\|\Delta \tilde{t}-S \delta t\|+\beta_{1}\|\delta t\|+\beta_{2}\|L \delta t\|
$$

where $S$ is GTT sensitivity of reservoir response to GDM parameters given by Eq. 7.2. Since the gradually deformed model honors static data and covariance structure, there is no need to have the norm or roughness constraints. The greatly reduced size of the problem also negates the need for regularization measures. The new deterministic objective function is now reduced to the following form

$$
O b j=\|\Delta \tilde{t}-S \delta t\|
$$

which is essentially fitting the response to observed data. The system of equations to be solved is now simply,

$$
S \delta t=\Delta \tilde{t}
$$

The system of linear equations given by Eq. 7.5 does not depend on the size of the model but on the number of GDM parameters. In regular GTT inversion, if we have $J$ wells and a model of size $M$ then the size of $S$ is $J^{*} M$. Adding the additional constraints will make the size of the linear system of equations to be solved of size $(J+2 * M) * M$. For GTT-GDM, however, the size of the linear system of equations given by Eq. 7.5 is only $J * K$ where $K$ is the number of GDM parameters regardless of model size. Eq. 7.5 can be 
solved using any of the iterative methods available in literature due to its relatively small size. In our work, however, we used the $l s q r^{35}$ method due to its robustness.

\subsection{D-Example: 9-Spot Water Flood}

To illustrate the method, we used a 2D 9-spot water flood model with a mesh size of 21x21. The reference and prior models are shown in Figure 7.1. The reference model was created by randomly deforming 100 unconditional stochastic models generated using LU simulation (LUSIM) and a spherical variogram model with a range of $30 \mathrm{ft}$ and no nugget effect. The prior model was generated by randomly deforming a subset of 25 stochastic models which are also used for global and local GTT-GDM inversion. Normal GTT inversion using the same prior model has been performed for comparison.
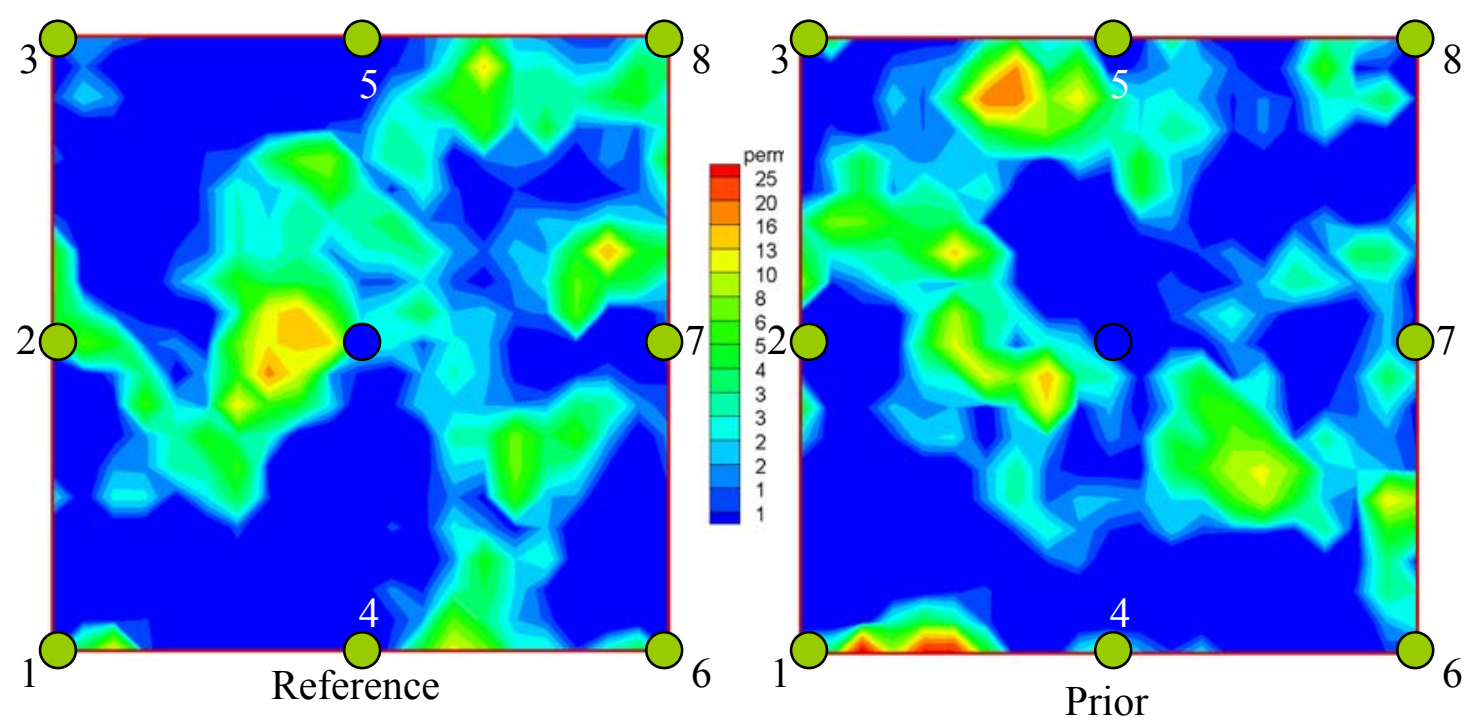

Figure 7.1 - Reference and prior models 
During GTT-GDM inversion, we gradually deformed the standard normal deviates and then used LU simulation with Cholesky decomposition to impose structure. Deformed models were then converted from standard normal deviates to permeability values using lognormal transform with a $\log$ mean of -0.093 and standard deviation of 1.36. LU simulation, or any other simulation method that separates structure from stochastic components, is needed for local GDM to avoid surface discontinuities between regions. For large problems, the LU algorithm can be expensive and other efficient methods like FFT-MA ${ }^{62}$ can be used instead. Figure 7.2 shows regular GTT inversion match.

Most wells achieved a good match except well\#4 due the prior model being substantially different than the reference model in the area around it. Most of the change is around well\#8 which achieved an excellent match. Regular GTT used the deterministic formulation with norm and roughness constraints to regularize the problem and produce a match that is close to the prior and exhibit smoothness that help reproduce large scale features. Figure 7.3 shows global GTT-GDM inversion match where the whole model is deformed during iterations. The match is not as good as regular GTT due to the structure constraints and the inflexibility of global GDM albeit almost all wells show an improved match. The matched model honors the structure which imposes a constraint on the problem that is stricter than the norm and roughness constraints used in regular GTT. Norm and roughness constraints penalize the objective function when values move away from the constrained direction while the structure constraint does not allow moving in any direction except if it honors the imposed covariance structure which is achieved by design. Local GDM gives more flexibility by independently deforming local regions which makes it possible to maintain a match in a local area while deforming other parts of the model. Figure 7.4 shows the results obtained with local GTT-GDM which is close to the level of water cut match obtained from regular GTT method. The final model reproduces the structure by design while matching the performance of regular GTT. In Figure 7.5, we compare RMS error reduction using the three methods. Regular GTT converges smoothly due to the regularization constraints imposed. Different norm and roughness weights were tested and the run shown is for the best match. Global GTTGDM fluctuates due to its relative inflexibility where a good match in a specific area can 
be lost when the whole model is deformed. This is less of a problem with local GTTGDM where a good local match is maintained while other parts of the model are being perturbed leading to better convergence and over all match as shown in Figure 7.5.

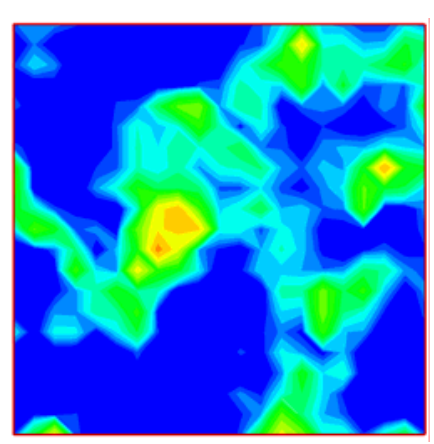

Reference
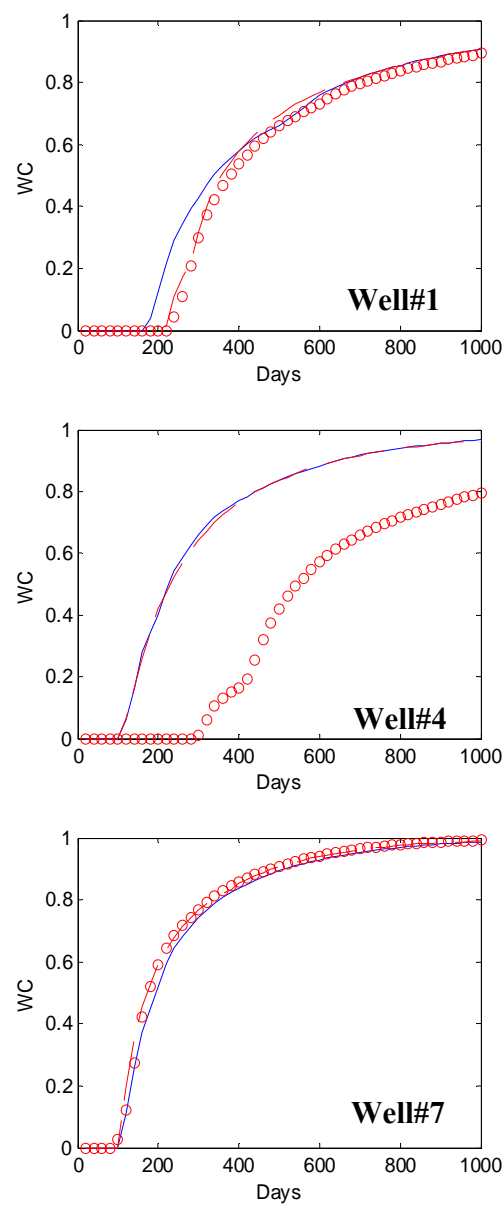

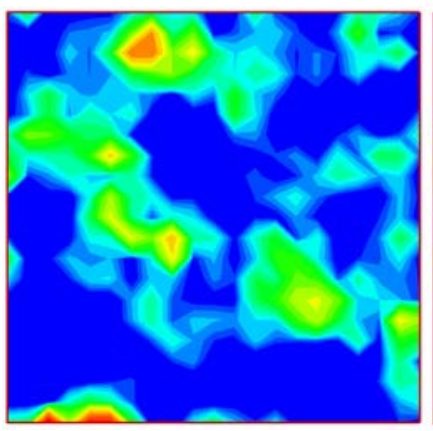

Prior
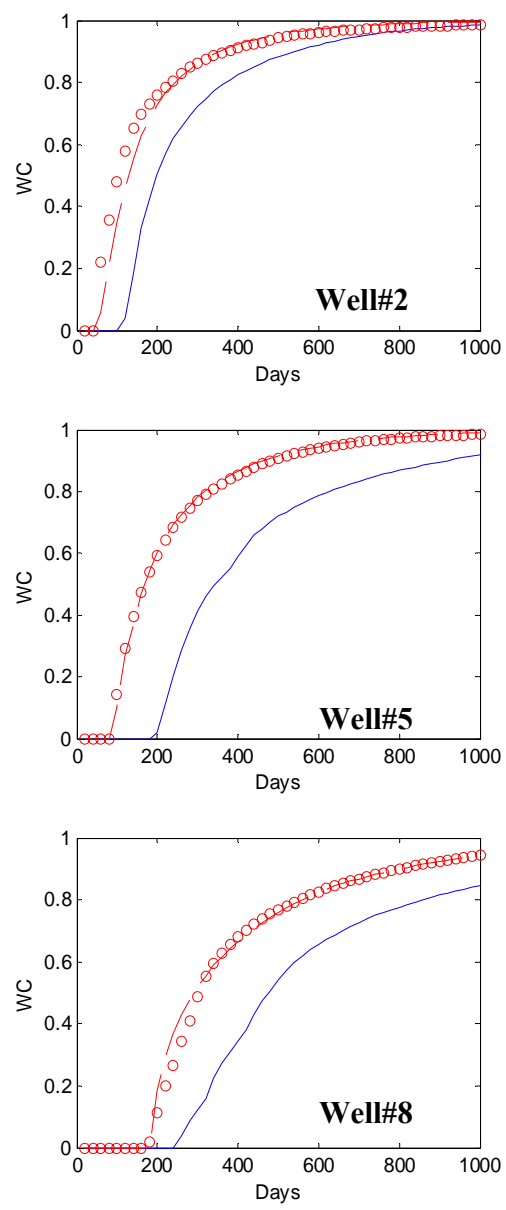

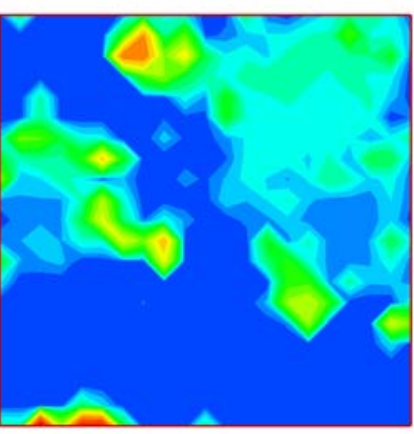

Match
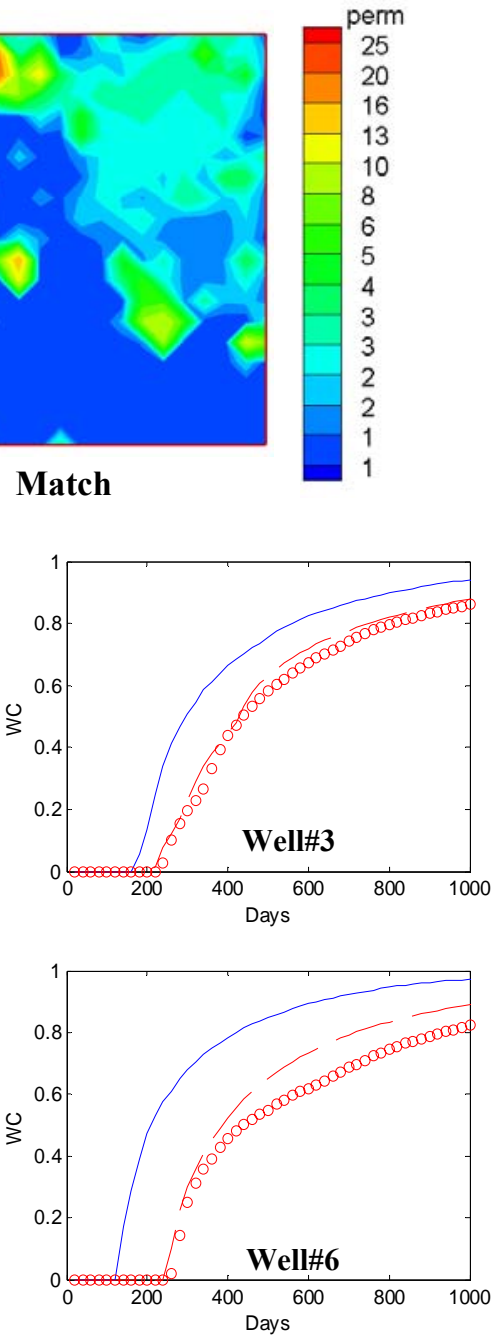

Figure 7.2 - Regular GTT inversion match 


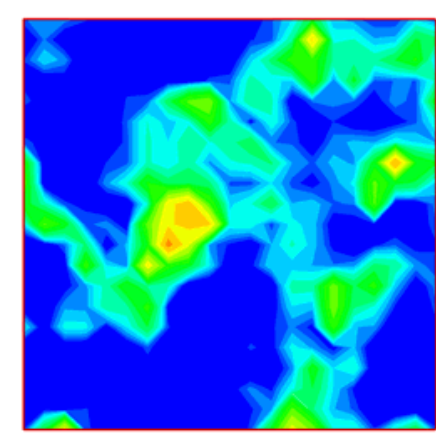

Reference
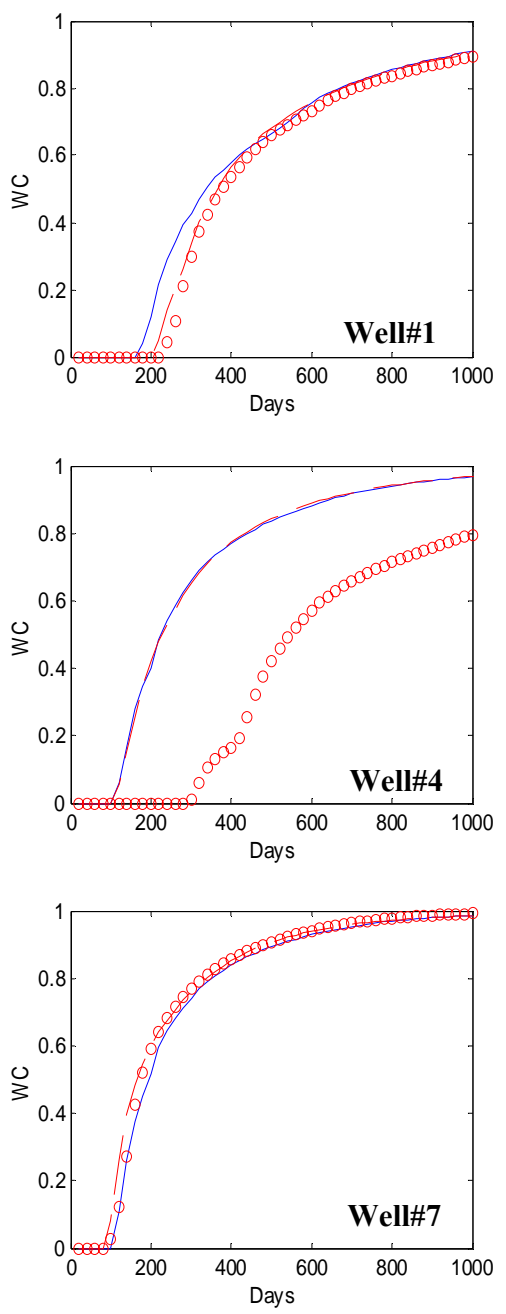

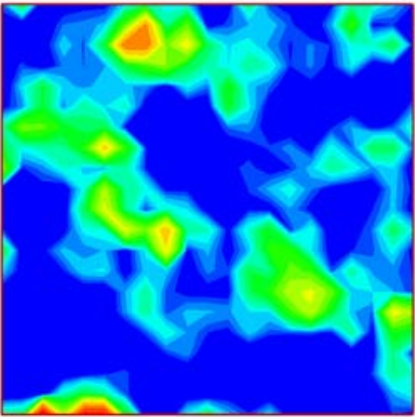

Prior
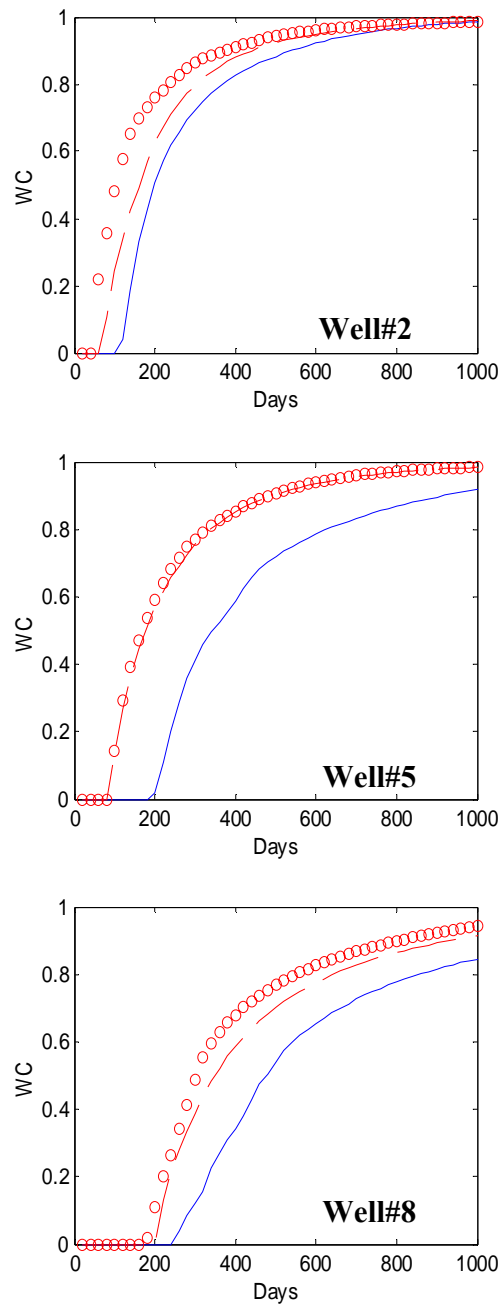

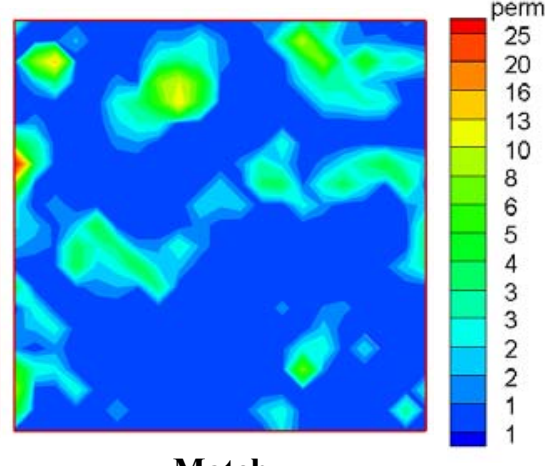

Match
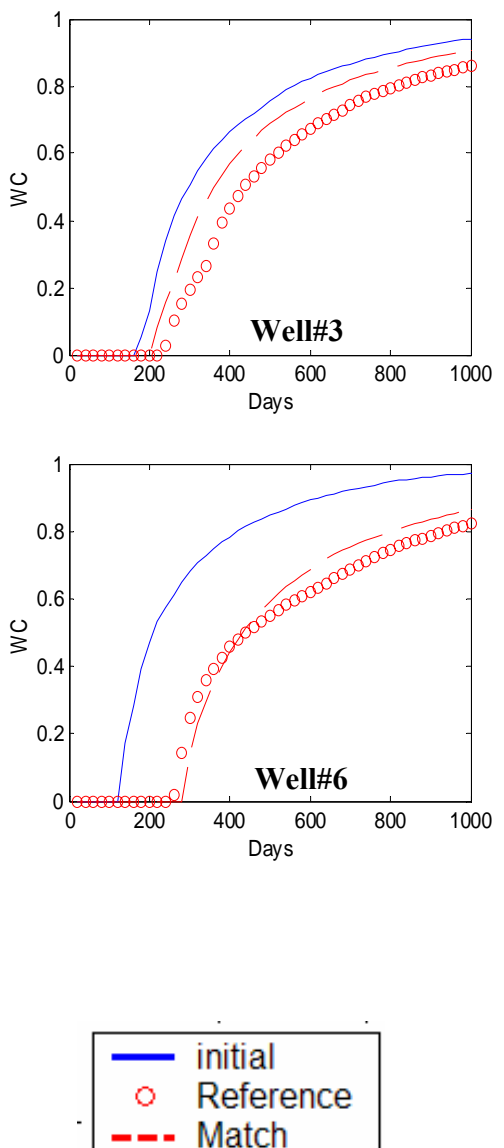

Figure 7.3 - Global GTT-GDM inversion match 


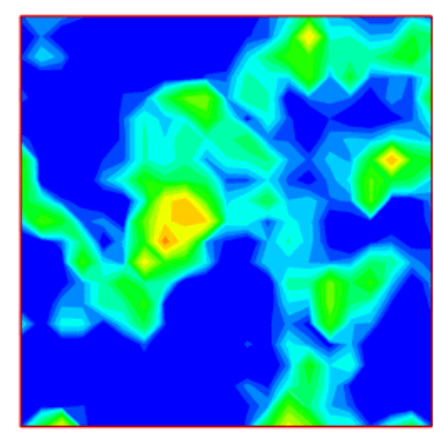

Reference
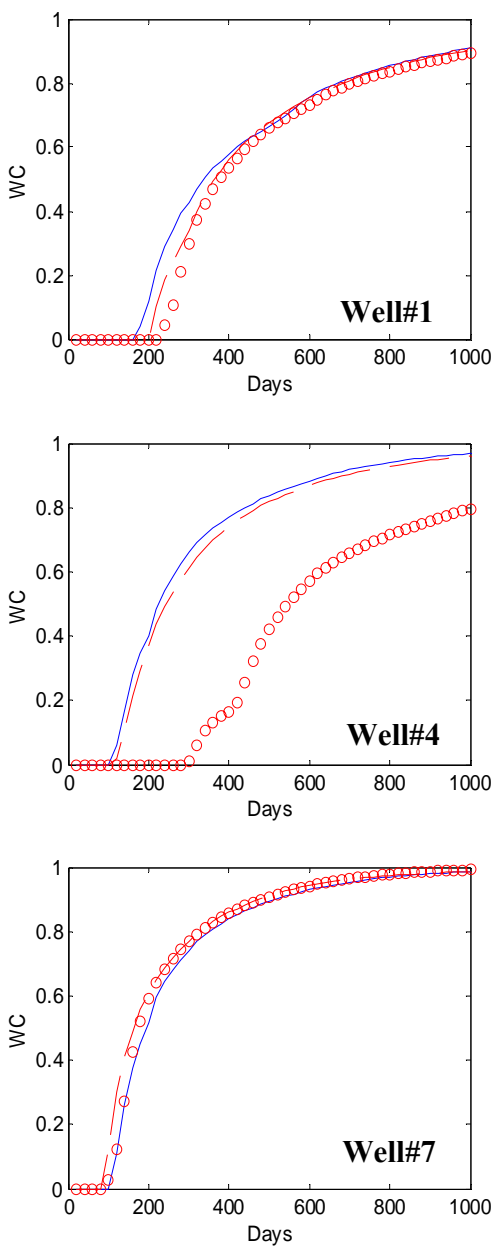

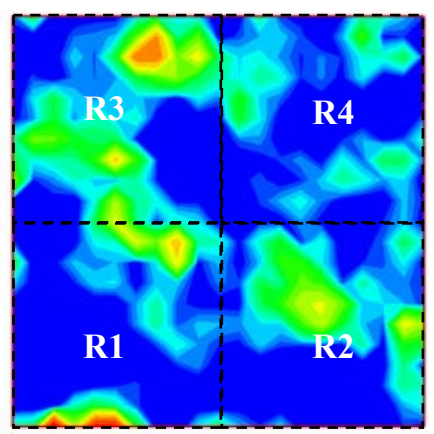

Prior
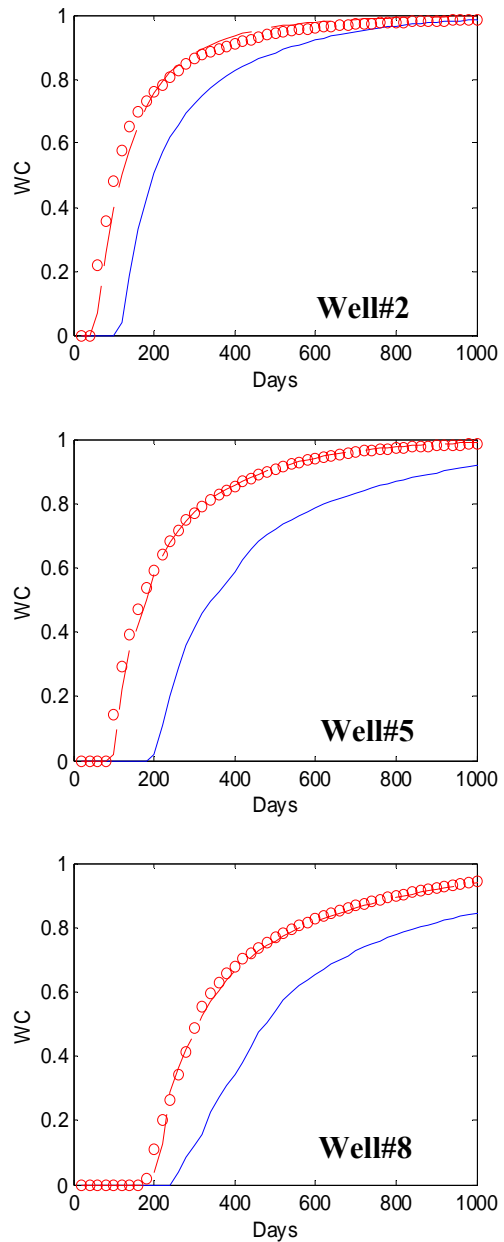

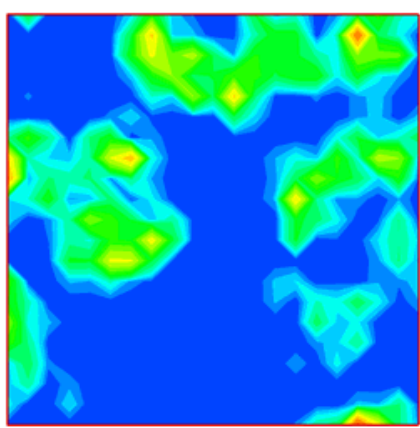

Match
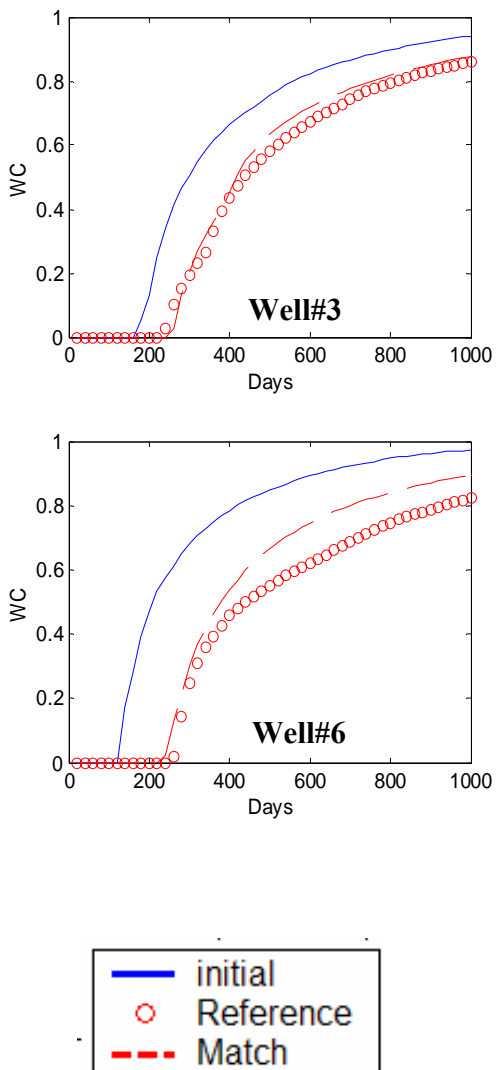

Figure 7.4 - Local GTT-GDM inversion match using four local regions 


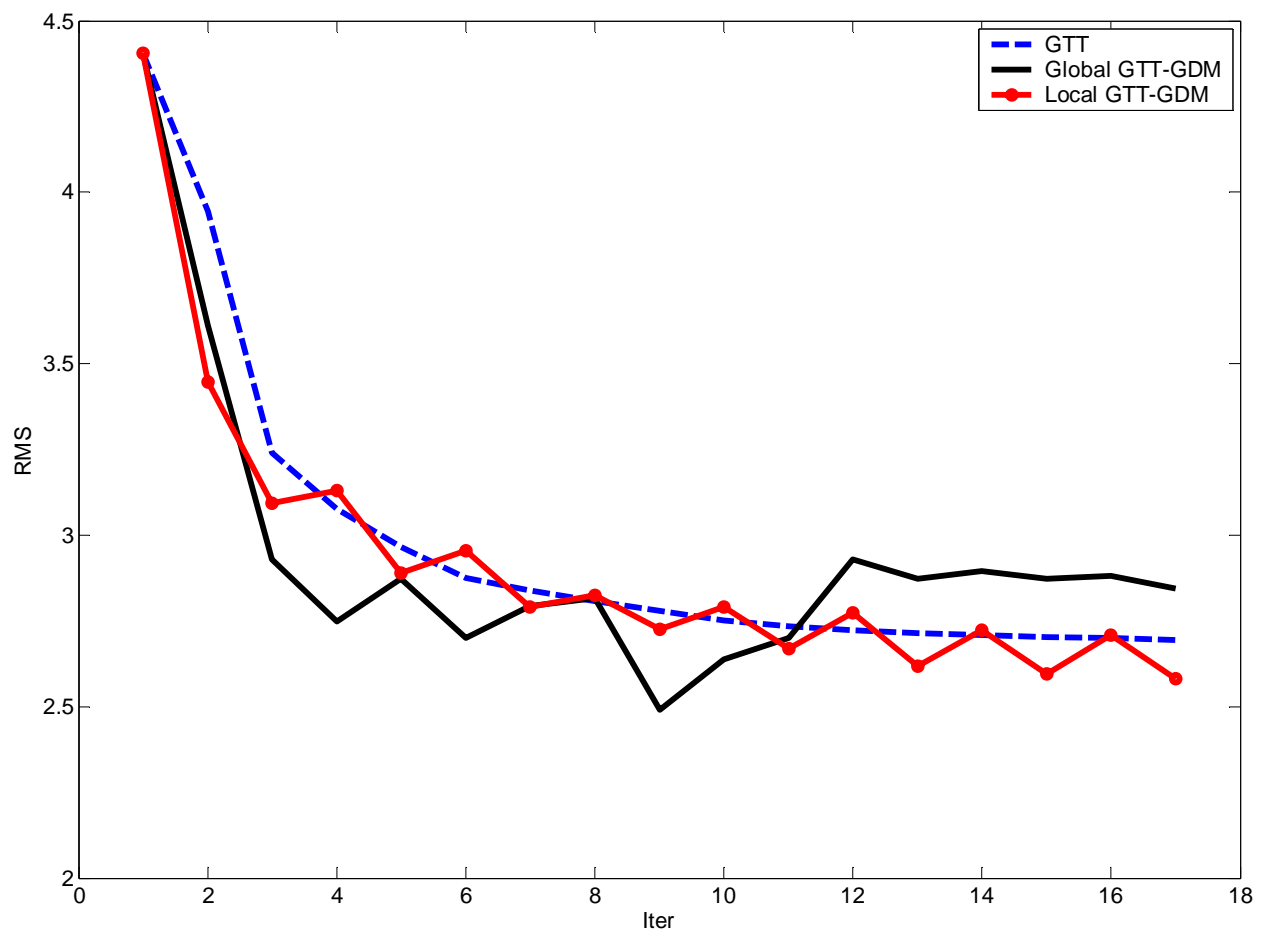

Figure 7.5 - RMS reduction comparison

The performance of local GTT-GDM is equivalent to regular GTT in this particular problem while giving a better model in terms of honoring the geological structure. In cases where structure parameters are not known or there is a large uncertainty associated with them, we can include them as unknown fitting parameters. Sensitivity to structural parameters (e.g. correlation range) can be calculated numerically and one possible approach is to use two nested iteration loops for deformation parameters and structural parameters. 


\section{CHAPTER VIII \\ CONCLUSIONS AND RECOMMENDATIONS}

\subsection{Conclusions}

Integration of dynamic data in petroleum reservoirs is a challenging endeavor, yet critical for reservoir management and development. The existence of natural fractures imposes an extra challenge due to their high impact on fluid sweep and recovery. The need to integrate dynamic data into naturally fractured reservoirs has gained greater attention recently as data collected from mature fields suggest that fractures are playing a greater role in conditioning fluid flow than previously thought.

In this work, we have proposed a streamline-based production data integration technique for naturally fractured reservoirs using the dual porosity approach. The principal features of our method are the extension of streamline-derived analytic sensitivities to account for matrix-fracture interactions and the use of our previously proposed generalized travel time inversion for history matching. Our proposed workflow has been demonstrated by using both a dual porosity streamline simulator and a commercial finite difference simulator. The approach is computationally efficient and well suited for large scale field applications in naturally fractured reservoirs with changing field conditions. The use of the generalized travel time concept enabled us to match both the breakthrough and amplitude of the reference response in one step. As a result, the method has proven to be well-suited for large scale field applications under diverse conditions and can be applied routinely to integrate dynamic data during reservoir characterization and management.

While the integration of dynamic data produces a conditioned model that could be used for forecasting reservoir response, assessing the uncertainty associated with such a response is critical to risk analysis and future development studies. Assessing uncertainty involves sampling the reservoir parameter a posterior probability density function that is 
conditioned to both static and dynamic data. Rigorous sampling methods like MCMC are known to give correct distributions but require full simulation run for every proposal made which can be extremely expensive for high resolution models. In this work, we have formulated a streamline-based approximate MCMC method that achieves a high acceptance rate while preserving the robustness of the traditional MCMC method. Streamline-based sensitivities are used to approximate the likelihood in the MetropolisHastings algorithm and streamline or finite difference simulators can be used in our workflow to calculate the exact likelihood. Such flexibility lends itself very well to a broad range of field applications and conditions and can be integrated in risk analysis studies with minor modification to existing workflows.

The specific conclusions of our study are summarized as follows.

\section{- On streamline-based dynamic data integration in naturally fractured reservoirs}

1. Streamline-based analytic sensitivity computations have been extended to naturally fractured reservoirs using the dual porosity approach. The matrix-fracture interactions are accounted for using predictor-corrector steps that involve convection along streamline followed by matrix-fracture exchange.

2. A comparison of the streamline-based sensitivities with those computed using the numerical perturbation method shows close agreement, indicating the validity of our approach. The streamline-based sensitivity computation is extremely efficient and requires a single forward simulation.

3. We have used the streamline-derived sensitivities in conjunction with a previously proposed generalized travel time inversion for integration of production data in fractured reservoirs. The generalized travel-time inversion is robust, computationally efficient and eliminates much of the time-consuming trial-and-error associated with manual history matching.

4. We have combined the streamline-derived sensitivities with a dual porosity finitedifference simulator to exploit the efficiency of the streamline approach and the versatility of the finite-difference simulator. Use of finite-difference simulation 
allows us to include compressibility effects, strong matrix fracture coupling and cross-streamline mechanisms.

5. We have demonstrated the power and efficiency of our proposed method using 2-D and 3-D examples designed after realistic field conditions. For the 3-D application, the results indicate the role of production data and prior information in terms of reproducing the fracture connectivity and fluid flow response in the reservoir.

\section{- On streamline-based uncertainty assessment using approximate MCMC formulation}

1. A streamline-based approximate MCMC method has been formulated. The method shares the robustness of traditional MCMC methods while reducing significantly the computational cost involved.

2. The proposed method is computationally efficient and can handle high resolution models under diverse field operating conditions which lend itself very well to a broad range of practical field applications.

3. Streamline or finite difference simulators are used in our work flow which makes the method applicable to existing risk analysis workflows with minor modifications.

4. The method requires a single simulation run to obtain both reservoir response and reservoir parameter sensitivities which substantially reduce the computational cost involved.

5. Since the method performs an exact likelihood calculation for all proposals accepted by the approximate likelihood step using the same acceptance probability level, the method does not compromise the rigorousness experienced with the traditional MCMC method.

6. The efficiency of our proposed method has been demonstrated using both 2-D and 3D examples with realistic field conditions. The method maintained high acceptance and convergence rates when compared with the full MCMC method which translates into substantial cost savings for high resolution models. 
- On generalized travel time inversion with gradual deformation method (GTTGDM)

1. Analytical streamline-based sensitivities are derived for the gradual deformation parameters. Analytical sensitivities can be calculated using a single simulation run for both global and local deformation parameters.

2. Current GDM inversion methods are limited to a combination of only two or three models due to the computational cost of numerical sensitivities which leads to slow convergence and limited search direction. Our method is completely general and can be used for any number of basis models which leads to faster convergence and much broader search direction. Since the method requires only a single simulation run, it is much faster than even the basic GDM inversion which combines two models and requires two simulation runs to calculate numerical sensitivities.

3. Local GDM leads to better convergence compared to global GDM. Its ability to perturb regions independently from each other leads to better overall match.

4. Using GDM and generalized travel time match reduces the size of the problem significantly. The number of system of linear equations to be solved is equal to the number of wells while the number of unknowns is equal to the number of GD parameters regardless of model size and number of data points.

5. The performance of the method has been demonstrated using a 2D 9-spot water flood. The method performance was comparable to regular GTT for this particular problem while producing a match that preserved the geological structure.

6. For large problems with local GDM, methods more efficient than LU simulation should be used. One area to investigate is using the stencil based algorithm for calculating covariance matrix inverse which can improve LU simulation efficiency for large problems. 


\subsection{Recommendations}

The integration of field data in reservoir characterization studies is still an active research area. We have extended proven dynamic data integration methods to fractured reservoirs using the dual porosity single permeability (DPSP) formulation which is widely used to model naturally fractured reservoirs. In situations where fluids flow in both the matrix and the fracture systems, the dual porosity dual permeability (DPDP) formulation should be used and the joint sensitivities for both systems need to be derived. The complex interaction between the two systems and its effect on sensitivity should be further explored.

Current formulation for the streamline-based sensitivity is currently limited to twophase water/oil flow. While such formulation works well in most of the pressure maintenance cases, it needs to be extended for cases with significant gas production. Three-phase flow streamline simulation is still an active research area and deriving streamline-based sensitivities for such systems will be required before extending dynamic data integration methods to such cases.

Uncertainty assessment methods are still an active research area due to the high cost associated with such methods and the need to evaluate reservoir forecast uncertainty in risk analysis studies. The streamline-based approximate MCMC method demonstrated a promising potential and further research should explore the optimization of important parameters that affect efficiency such as step size.

The gradual deformation method is an excellent framework to honor structural parameters if such parameters can be extracted reliably from geologic and seismic data. The inefficiency of the GDM method is largely due to the use of numerical sensitivities which make the method restricted to deforming two or three basis models at a time. In our work, the derivation of fast streamline-based analytical sensitivities and the use of generalized travel time inversion have obtained a performance in par with the more robust GTT inversion while honoring the geological structure. Local GDM should be used whenever possible to obtain a better overall match and achieve faster convergence. 


\section{NOMENCLATURE}

\begin{tabular}{|c|c|}
\hline$\Delta \widetilde{t}$ & $=$ Vector of generalized travel time shift \\
\hline$\tau$ & $=$ Time of flight \\
\hline$\alpha_{i, j}$ & $=$ Probability of accepting transition in the Markov chain \\
\hline$\mu_{\mathrm{m}}$ & $=$ Viscosity of $(\mathrm{m})$ phase, $\mathrm{m}$ stands for oil and water \\
\hline$\gamma_{\mathrm{m}}$ & $=$ Specific weight of $(\mathrm{m})$ phase, $\mathrm{m}$ stands for oil and water \\
\hline$\rho_{\mathrm{m}}$ & $=$ Density of phase $\mathrm{m}, \mathrm{m}$ stands for oil and water \\
\hline$\rho_{\mathrm{msc}}$ & $=$ Density of phase $\mathrm{m}$ at standard conditions \\
\hline$\lambda_{t}$ & $=$ Total mobility ratio \\
\hline$\Delta t$ & $=$ Time step \\
\hline$\Delta t_{j}$ & $=$ Time shift at well $j$ \\
\hline$\Delta x_{i}, \Delta y_{j}, \Delta z_{k}$ & $=$ Cartesian grid block sizes \\
\hline $\mathrm{C}_{\mathrm{d}}$ & $=$ Data covariance matrix \\
\hline $\mathrm{C}_{\mathrm{M}}$ & $=$ Prior covariance matrix of the model parameter \\
\hline $\mathrm{D}$ & $=$ Depth \\
\hline $\mathrm{d}_{\mathrm{obs}}$ & $=$ Column vector with observed data \\
\hline$f\left(d_{o b s}\right)$ & $=$ Marginal probability distribution \\
\hline$f\left(d_{o b s} / m\right)$ & $=$ Likelihood probability distribution given the prior distribution \\
\hline$f(m)$ & $=$ Prior probability distribution \\
\hline$f\left(m / d_{o b s}\right)$ & $=$ Posterior probability distribution given the observed data \\
\hline$F_{w}$ & $=$ Fractional flow of water \\
\hline$g(m)$ & $=$ Column vector with calculated reservoir performance data \\
\hline$S$ & $=$ Sensitivity matrix \\
\hline$I$ & $=$ Identity matrix \\
\hline$K$ & $=$ Permeability \\
\hline$K_{r m}$ & $=$ Relative permeability to phase $(\mathrm{m}) ; \mathrm{m}$ stands for oil or water \\
\hline$M$ & $=$ Number of model parameters \\
\hline$m$ & $=$ Column vector of the reservoir parameter \\
\hline MAP & $=$ Maximum $a$ posteriori estimate \\
\hline$M C$ & $=$ Markov chain \\
\hline$M C M C$ & $=$ Markov chain Monte Carlo \\
\hline$m_{p}$ & $=$ Column vector with prior knowledge of reservoir parameter \\
\hline$n_{d}$ & $=$ Number of data points \\
\hline$N_{d}$ & $=$ Number of data points \\
\hline$N_{d j}$ & $=$ Number of data points at well $\mathrm{j}$ \\
\hline$n_{w}$ & $=$ Number of wells \\
\hline$N_{w}$ & $=$ Number of wells \\
\hline$N_{x}, N_{y}, N_{z}$ & $=$ Number of grid blocks in the $\mathrm{x}, \mathrm{y}$, and $\mathrm{z}$ direction \\
\hline $\operatorname{Obj}(m)$ & $=$ Objective function of Bayesian formulation \\
\hline$P$ & $=$ Pressure \\
\hline$\phi$ & $=$ Porosity \\
\hline
\end{tabular}




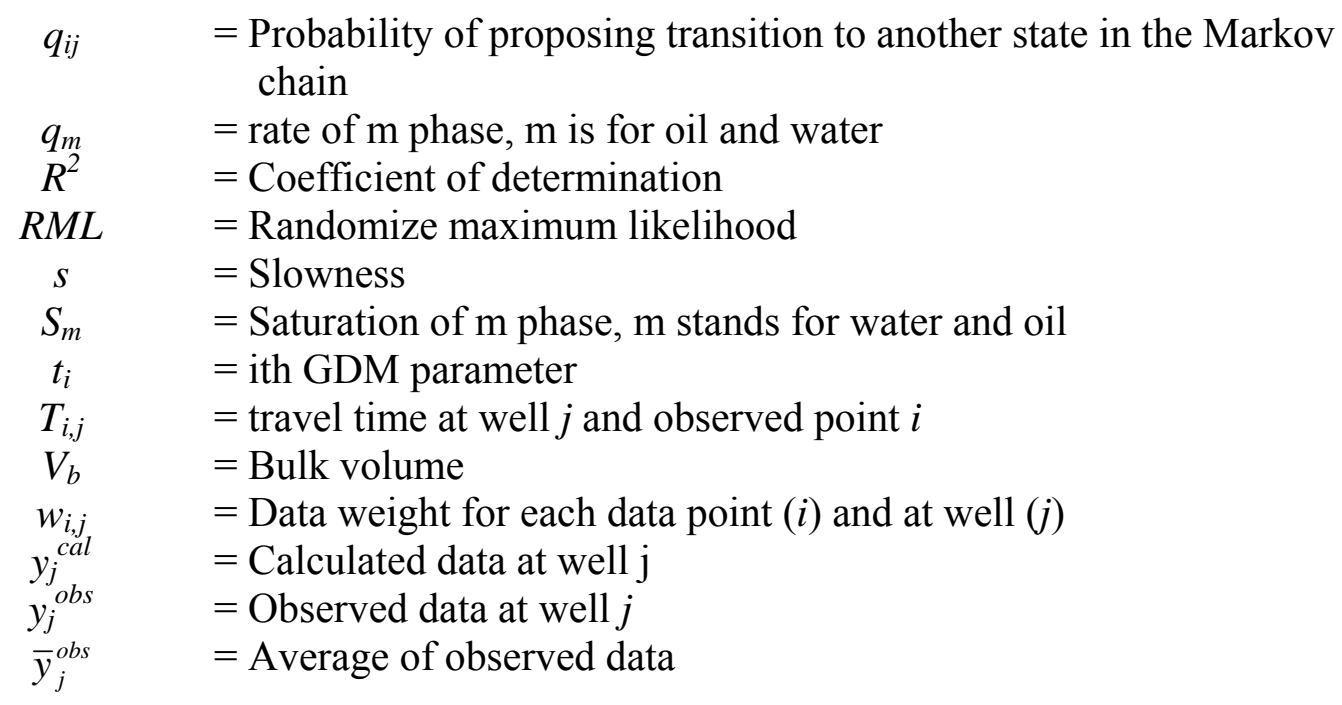




\section{REFERENCES}

1. Nelson, R. A.: Geological Analysis of Naturally Fractured Reservoirs, Gulf Publishing Company, Houston (1985).

2. Dershowitz, W., Lapointe, P., Eiben, T. and Wei, L.: "Integration of Discrete Fracture Network Methods with Conventional Simulator Approaches," SPE Res. Eval. \& Eng., (April 2000), 165-170.

3. Tamagawa, T., Matsuura, T., Anraku, T., Tezuka, K. and Namikawa, T.: "Construction of Fracture Network Model Using Static and Dynamic Data," paper SPE 77741 presented at the 2002 SPE Annual Technical Conference and Exhibition, San Antonio, TX, Sept. 29-Oct. 2.

4. Long, J. C. S., Doughty C., Datta-Gupta, A., Hestir, K. and Vasco, D. W.: "Component Characterization: An Approach to Fracture Hydrogeology," in Subsurface Flow and Transport: A Stochastic Approach, G. Dagan and S. P. Neuman (Eds.), Cambridge University Press, Cambridge, UK (1997), 179-195.

5. Datta-Gupta, A., Vasco, D. W. and Long, J. C. S.: "Detailed Characterization of a Fractured Limestone Formation Using Stochastic Inverse Approaches," SPEFE (September 1995) 133; Trans., AIME, 299.

6. Vasco, D.W., Yoon, S., and Datta-Gupta, A.: "Integrating Dynamic Data into HighResolution Reservoir Models Using Streamline-Based Analytic Sensitivity Coefficients," SPEJ (December 1999) 389.

7. He, Z., Datta-Gupta, A., and Yoon, S.: "Streamline-Based Production Data Integration with Gravity and Changing Field Conditions," SPEJ (December 2002), 423.

8. Qassab, H., Khalifa, M., Pavlas, R., Khargoria, A., He, Z., Lee, S. H. and DattaGupta, A.: "Streamline-Based Production Data Integration Under Realistic Field Conditions: Experience in a Giant Middle-Eastern Oil Reservoir,” paper SPE 84079 presented at the 2003 SPE Annual Technical Conference and Exhibition, Denver, CO, 5-8 October. 
9. Wang, Y. and Kovscek, A.R.: "A Streamline Approach to History Matching Production Data," paper SPE 59370 presented at the 2000 SPE/DOE Symposium on Improved Oil Recovery, Tulsa, 3-5 April.

10. Milliken, W.J., Emanuel, A.S. and Chakravarty, A.: "Application of 3-D Streamline Simulation to Assist History Matching," paper SPE 63155 presented at the 2000 SPE Annual Technical Conference and Exhibition, Dallas, 1-4 October.

11. Barenblatt, G.E., Zheltov, I. P., and Kochina, I.N.: "Basic Concepts in the Theory of Seepage of Homogenous Liquids in Fissured Rocks," J. Appl. Math. and Mech. Eng. Transl. (1960), 1286.

12. Kazemi, H., Merrill L.S. Jr., Porterfield, K.L. and Zeman, P.R.: "Numerical Simulation of Water-Oil Flow in Naturally Fractured Reservoirs," SPEJ (December 1976) 317: Trans., AIME 261.

13. Dean, R.H. and Lo, L.L.: "Simulations of Naturally Fractured Reservoirs," SPERE (May 1988) 638.

14. Di Donato, G., Huang, W., and Blunt, M.J.: "Streamline-Based Dual Porosity Simulation of Fractured Reservoirs," paper SPE 84036 presented at the 2003 Annual Technical Conference and Exhibition, Denver, CO, 5-8 October.

15. Al-Huthali, A.H.: "Streamline Simulation of Counter-Current Imbibition in Naturally Fractured Reservoirs," Journal of Petroleum Science and Engineering, 2005 (in press).

16. Cheng, H., Khargoria, A., He, Z. and Datta-Gupta, A.: "Fast History Matching of Finite-Difference Models Using Streamline-Derived Sensitivities,” paper SPE 89447 presented at the 2004 SPE/DOE Fourteenth Symposium on Improved Oil Recovery, Tulsa, 17-21 April.

17. Oliver, D.S., He, N. and Reynolds, A.C.: "Conditioning Permeability Fields to Pressure Data," presented at the $19965^{\text {th }}$ European Conference on the Mathematics of Oil Recovery, Leoben, Austria, September 3-6.

18. Liu, N., Betancourt, S., and Oliver, D.S.: "Assessment of Uncertainty Assessment Methods," paper SPE 71624 presented at the 2001 SPE Annual Technical Conference and Exhibition, New Orleans, 30 September-3 October. 
19. Tarantola, A.: Inverse Problem Theory - Methods for Data Fitting and Model Parameters Estimation, Elsevier, New York (1987).

20. Fox, C. and Nicholls, G.: "Sampling Conductivity Images via MCMC." in The Art and Science of Bayesian Image Analysis, K.V. Mardia, C.A. Gill, R.G. Aykroyd (eds.), Proceedings of the Leeds Annual Statistical Research Workshop (LASR), Leeds University Press, Leeds, UK (July 1997), 91-100.

21. Wu, Z. and Datta-Gupta, A.: "Rapid History Matching Using a Generalized Travel Time Inversion Method," SPEJ (June 2002) 113.

22. Cheng, H., Wen, X., Milliken, W. and Datta-Gupta, A.: "Field Experiences with Assisted and Automatic History Matching Using Streamline Models," paper SPE 89857 presented at the 2004 SPE Annual Technical Conference and Exhibition, Houston, 26-29 September.

23. King, M. J. and Datta-Gupta, A.: "Streamline Simulation: A Current Perspective," In Situ (1998) 22, No.1, 91.

24. Datta-Gupta, A. and King, M. J.: "A Semi-Analytic Approach to Tracer Flow Modeling in Heterogeneous Permeable Media," Advances in Water Resources (1995), $18,9$.

25. Kazemi, H., Gilman, J.R. and Elsharkawy, A.M.: "Analytical and Numerical Solution of Oil Recovery from Fractured Reservoirs with Empirical Transfer Functions," SPEJ (May 1992) 219.

26. Rossen, R.H. and Shen, E.I.: "Simulation of Gas/Oil Drainage and Water/Oil Imbibitions in Naturally Fractured Reservoirs," paper SPE 16982 presented at the 1987 SPE Annual Technical Conference and Exhibition, Dallas, 27-30 September.

27. Bratvedt, F., Gimse, T. and Tegnander, C.: "Streamline Computations for Porous Media Flow Including Gravity," Transport in Porous Media (1996), 25, 63.

28. Pollock, D. W.: "Semianalytical Computation of Pathlines for Finite-Difference Models," Groundwater (1988), 26, No. 6, 743.

29. Bissel, R.C., Killough, J.E. and Sharma, Y.: "Reservoir History Matching Using the Method of Gradients," paper SPE 24265 presented at the 1992 SPE European Petroleum Computer Conference, Stavanger, Norway, 25-27 May. 
30. Landa, J.L. and Horne, R.N.: “A Procedure to Integrate Well Test Data, Reservoir Performance History and 4-D Seismic Information into a Reservoir Description," paper SPE 38653 presented at the 1997 SPE Annual Technical Conference and Exhibition, San Antonio, TX, 5-8 October.

31. Wen, X., Deutsch, C. and Cullick, A.S.: "High Resolution Reservoir Models Integrating Multiple-Well Production Data,” paper SPE 38728 presented at the 1997 SPE Annual Technical Conference and Exhibition, San Antonio, TX, 5-8 October.

32. Reynolds, A. C., He, N., and Oliver, D.S.: "Reducing Uncertainty in Geostatistical Description with Well Testing Pressure Data," Proc., International Reservoir Characterization Conference, Houston (1997) 443-457.

33. Anterion, F., Eymard, R. and Karcher, B.: "Use of Parameter Gradients for Reservoir History Matching," paper SPE 18433 presented at the 1989 SPE Symposium on Reservoir Simulation, Houston, 6-8 February.

34. Vega, L., Rojas, D. and Datta-Gupta, A.: "Scalability of the Deterministic and Bayesian Approaches to Production Data Integration into Field-Scale Reservoir Models," paper SPE 79666 presented at the 2003 SPE Reservoir Simulation Symposium, Houston, 3-5 February.

35. Paige, C.C. and Saunders, M. A.: "LSQR: An Algorithm for Sparse Linear Equations and Sparse Least Squares," ACM Trans. Math. Software (1982), 8, No. 1, 43.

36. Vega, L.: “An Efficient Bayesian Formulation for Production Data Integration into Reservoir Models," Ph.D. Dissertation, Texas A\&M University, College Station, TX (2003).

37. Guerreiro, L., Costa Silva, A., Alcobia, V. and Soares, A.: "Integrated Reservoir Characterization of a Fractured Carbonate Reservoir," paper SPE 58995 presented at the 2000 SPE Inter. Petroleum Conf. and Exhib., Villahermosa, Mexico, 1-3 February.

38. GeoQuest: ECLIPSE User Guide, Vers. 2003A, Schlumberger, Houston (2003).

39. Oliver, D. S., Reynolds, A. C., Bi, Z. and Abacioglu, Y.: "Integration of Production Data into Reservoir Models,” Petroleum Geoscience (2001) 7, S65. 
40. Liu, N., Betancourt, S., and Oliver, D.S.: “Assessment of Uncertainty Assessment Methods," paper SPE 71624 presented at the 2001 SPE Annual Technical Conference and Exhibition, New Orleans, 30 September-3 October.

41. Omre, H., Tjelmeland, H., and Wist, H. T.: "Uncertainty in History Matching Model Specification and Sampling Algorithms," Technical Report Statistics No. 6/1999, Department of Mathematical Sciences, Norwegian University of Science \& Technology, Trondheim, Norway (1999).

42. Oliver, D. S., Cunha, L. B., and Reynolds, A. C.: "Markov Chain Monte Carlo Methods for Conditioning a Permeability Field to Pressure Data," Mathematical Geology (1997), 29, No. 1, 61.

43. Cunha, L. B., Oliver, D. S., Rednar, R. A., and Reynolds, A. C.: "A Hybrid Markov Chain Monte Carlo Method for Generating Permeability Fields Conditioned to Multiwell Pressure Data and Prior Information," SPEJ (1998) 3, No. 3, 261.

44. Kitanidis, P. K.: "Quasi-Linear Geostatistical Theory for Inversion,” Water Resour. Res. (1995), 31, No. 10, 2411.

45. Oliver, D. S., He, N. and Reynolds, A. C.: “Conditioning Permeability Fields to pressure data," Proc., $5^{\text {th }}$ European Conference for the Mathematics of Oil Recovery, Leoben, Austria (1996), 1-11.

46. De Marsily, G., Lavedan, G., Boucher, M. and Fasanino, G.: "Interpretation of Interference Tests in a Well Field Using Geostatistical Techniques to Fit the Permeability Distribution in a Reservoir Model," Geostatistics for Natural Resources Characterization (1984), Part 2, 831.

47. Brefort, B. and Pelce, V.: "Inverse Modeling for Compressible Flow - Application to Gas Reservoirs," Proc., $2^{\text {nd }}$ European Conference on the Mathematics of Oil Recovery, Paris, France (1990), 331-334.

48. LaVenue, A. M. and Pickens, J. F.: “Application of a Coupled Adjoint Sensitivity and Kriging Approach to Calibrate a Groundwater Flow Model," Water Resour. Res. (1992), 28, No. 6, 1543.

49. Glimm, J., Hou, S., Kim, H., Lee, Y., Sharp, D. et al.: "Risk Management for Petroleum Reservoir Production: A Simulation-Based Study of Prediction," Computational Geosciences (2001) 5, No. 3, 173. 
50. Omre, H. and Lodoen, O.: " Improved Production Forecasts and History Matching Using Approximate Fluid-Flow Simulators.” SPEJ (September 2004), 339.

51. Craig, P.S., Goldstein, M., Rougier, J.C. and Seheult, A.H.: "Bayesian Forecasting for Complex Systems Using Computer Simulators,” J. American Statistical Assn. (2001) 96, No. 454, 717.

52. Kennedy, M. and O'Hagan, A.: "Bayesian Calibration of Computer Models," J. of the Royal Statistical Soc. (2001), Series B, 63, 425.

53. Metropolis, N., Rosenbluth, M., Rosenbluth, A., Teller, A. and Teller, E.: "Equation of State Calculations by Fast Computer Machines," Journal of Chemical Physics (1953) 21, 1087.

54. Hu, L. Y.: "Gradual Deformation and Iterative Calibration of Gaussian-Related Stochastic Models" Mathematical Geology (2000), 32, No. 1, 87.

55. Hu, L. Y.: "Gradual Deformation of Non-Gaussian Stochastic Simulations" presented at the 2000 6th Geostatistics Congress, Cape Town, South Africa, April 10-14.

56. Journel, A. G., and Huijbregts, C. J.: Mining Geostatistics Academic Press, London (1978), 600.

57. Liu, N. and Oliver, D.: "Experimental Assessment of Gradual Deformation Method," Mathematical Geology (2004), 36, No. 1, 65.

58. Farmer, C. L.: “Numerical rocks,” Mathematics of Oil Recovery, P.R. King (ed.), Oxford University Press, Oxford, UK (1992), 437.

59. Datta-Gupta, A., Kulkarni, K. N., Yoon, S. S. and Vasco, D. W.: "Streamlines, Ray Tracing and Production Tomography: Generalization to Compressible Flow," Petroleum Geoscience (May 2001), 7, 75.

60. Wu, Z. and Datta-Gupta, A.: "Rapid History Matching Using a Generalized Travel Time Inversion Method,” paper SPE 66352 presented at the 2001 SPE Reservoir Simulation Symposium, Houston, February 11-14.

61. Ravalec-Dupin, M. and Noetinger, B.: "Optimization with the Gradual Deformation Method" Mathematical Geology (2002), 34, No. 2, 125.

62. Le Ravalec M., Noetinger B. and Hu L.-Y.: "The FFT Moving Average (FFT-MA) Generator: An Efficient Numerical Method for Generating and Conditioning Gaussian Simulations," Mathematical Geology (2000), 32, No. 6, 701. 


\section{APPENDIX A}

\section{GENERALIZED TRAVEL TIME SENSITIVITIES FOR THE GRADUAL DEFORMATION METHOD}

Starting with the gradual deformation linear equation

$$
Y(x)=\sum_{i=0}^{M} \alpha_{i} Y_{i}(x)
$$

where the weights are given by following equation,

$$
\begin{aligned}
& \alpha_{0}=\prod_{i=1}^{m} \cos \left(t_{i}\right) \\
& \alpha_{i}=\sin \left(t_{i}\right) \prod_{j=i+1}^{m} \cos \left(t_{j}\right) \quad t \in(-\pi, \pi) \\
& \alpha_{m}=\sin \left(t_{m}\right)
\end{aligned}
$$

Combining Eq. A-1 and A-2,

$$
\begin{aligned}
Y=[ & \left.\prod_{i=1}^{m} \cos \left(t_{i}\right)\right] Y_{0} \\
& +\sum_{i=1}^{m-1}\left[\sin \left(t_{i}\right) \prod_{j=i+1}^{m} \cos \left(t_{j}\right)\right] Y_{i} \\
& +\sin \left(t_{m}\right) Y_{m}
\end{aligned}
$$

Sensitivity of parameter $Y$ to perturbation to parameter $t_{k}$ can be found by differentiating A-3 with respect to $t_{k}$. For example, if we differentiate with respect to $t_{3}$ we get the following expression, 


$$
\begin{aligned}
\frac{\partial Y}{\partial t_{3}}= & {\left[-\sin \left(t_{3}\right) \prod_{i=1}^{m} \cos \left(t_{i}\right)_{i \neq 3}\right] Y_{0} } & \leftarrow i=0 \\
& +\left[-\sin \left(t_{3}\right) \sin \left(t_{i}\right) \prod_{j=i+1}^{m} \cos \left(t_{j}\right)_{j \neq 3}\right] Y_{1} & \leftarrow i=1 \\
& +\left[-\sin \left(t_{3}\right) \sin \left(t_{i}\right) \prod_{j=i+1}^{m} \cos \left(t_{j}\right)_{j \neq 3}\right] Y_{2} & \leftarrow i=2 \\
& +\left[\cos \left(t_{3}\right) \prod_{j=k+1}^{m} \cos \left(t_{j}\right)\right] Y_{3} & \leftarrow i=3 \\
& +0 & \leftarrow i>3
\end{aligned}
$$

Examining Eq. A-4, we can construct a general expression for the sensitivity to any GDM parameter $t_{k}$

$$
\begin{aligned}
\frac{\partial Y}{\partial t_{k}}= & {\left[-\sin \left(t_{k}\right) \prod_{i=1}^{m} \cos \left(t_{i}\right)_{i \neq k}\right] Y_{0} } \\
& +\sum_{i=1}^{k-1}\left[-\sin \left(t_{k}\right) \sin \left(t_{i}\right) \prod_{j=i+1}^{m} \cos \left(t_{j}\right)_{j \neq k}\right] Y_{i} \\
& +\left[\cos \left(t_{k}\right) \prod_{j=k+1}^{m} \cos \left(t_{j}\right)\right] Y_{k}
\end{aligned}
$$




\section{VITA}

Mishal Habis Al Harbi was born in September 12, 1969. He received his B.S. from King Fahd University of Petroleum and Minerals, Saudi Arabia in 1994 and his M.S. from Stanford University, California in 1998, both in petroleum engineering.

He worked in Saudi Aramco reservoir management from 1994 to 1996 as a reservoir engineer where he served as a member of the initial development team of newly discovered Central Arabia fields. He also served from 1998 to 2000 in managing a number of producing fields in central Arabia and devising development strategies while serving in asset management and special projects teams. His work experience is in reservoir management, development strategy, reservoir characterization and field optimization.

He can be reached at:

P.O. Box 11695

Saudi Aramco

Dhahran, 31311

Saudi Arabia

Email: mishal_harbi@yahoo.com 